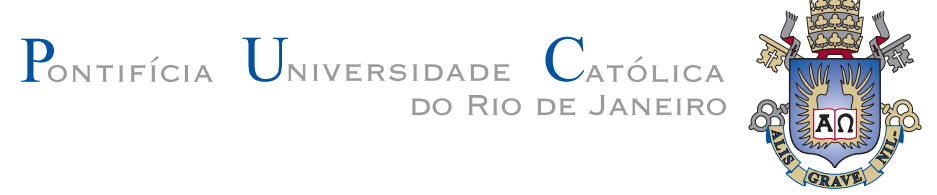

Alessandro Gaio Chimenton

Fluxos geodésicos Finsler transitivos e aplicações

Tese de Doutorado

Tese apresentada ao Programa de Pós-graduação em Matemática Aplicada da PUC-Rio como requisito parcial para obtenção do título de Doutor em Matemática Aplicada.

Orientador: Prof. Rafael Oswaldo Ruggiero Rodriguez 


\section{Fluxos geodésicos Finsler transitivos e} aplicações

Tese apresentada ao Programa de Pós-graduação em Matemática Aplicada da PUC-Rio como requisito parcial para obtenção do título de Doutor em Matemática Aplicada. Aprovada pela Comissão Examinadora abaixo assinada.

Prof. Rafael Oswaldo Ruggiero Rodriguez

Orientador

Departamento de Matemática - PUC-Rio

Prof. David Francisco Martinez Torres

Departamento de Matemática - PUC-Rio

Prof. Marcos Martins Alexandrino da Silva Instituto de Matemática e Estatística - USP

Prof. Miguel Angel Javaloyes Victoria Facultad de Matemática - Universidad de Murcia

Prof. Umberto Leone Hryniewicz Departamento de Matemática Aplicada - UFRJ

Prof. José Barbosa Gomes Departamento de Matemática - UFJF

Prof. José Eugenio Leal Coordenador Setorial do Centro Técnico Científico - PUC-Rio 
Todos os direitos reservados. É proibida a reprodução total ou parcial do trabalho sem autorização da universidade, do autor e do orientador.

\section{Alessandro Gaio Chimenton}

Graduou-se em Matemática - Licenciatura e Bacharelado pela Universidade Federal do Paraná em 2006. Obteve o título de Mestre em Matemática Aplicada em 2009 pela mesma universidade.

Ficha Catalográfica

Chimenton, Alessandro Gaio

Fluxos geodésicos Finsler transitivos e aplicações / Alessandro Gaio Chimenton; orientador: Rafael Oswaldo Ruggiero Rodriguez. - Rio de Janeiro : PUC-Rio, Departamento de Matemática, 2015.

v., 82 f: il. ; $29,7 \mathrm{~cm}$

1. Tese (doutorado) - Pontifícia Universidade Católica do Rio de Janeiro, Departamento de Matemática.

Inclui referências bibliográficas.

1. Matemática - Tese. 2. Visibilidade Finsler. 3. Fluxos geodésicos transitivos. 4. Grupos Gromov-hiperbólicos. 5. Superfícies Finsler k-básicas. I. Rodriguez, Rafael $O$. Ruggiero. II. Pontifícia Universidade Católica do Rio de Janeiro. Departamento de Matemática. III. Título. 


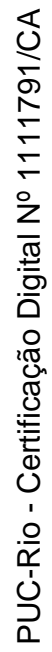

À Cleonice, minha mãe. 


\section{Agradecimentos}

Agradeço primeiramente ao meu orientador pelas precisas e importantes direções apontadas no decorrer da realização deste trabalho e pelas várias discussões, o que tornou possível obter uma visão clara das perguntas que respondemos neste trabalho. Obrigado também a Katia, Creuza, Orlando, Fernanda e Otavio, funcionários e ex-funcionários da secretaria do departamento de Matemática da PUC-Rio, pela inestimável, ágil e sempre presente ajuda no âmbito administrativo. Agradeço enfim aos professores e colegas estudantes do departamento e de outras instituições, particularmente ao Rodrigo Pacheco, José Victor, Otavio Kaminski, Ady Cambraia (alunos e ex-alunos da PUC-Rio) e Ricardo Paleari (IMPA) pelas excelentes discussões que auxiliaram a entender tanto pormenores importantes deste trabalho quanto conceitos de interesse geral. 


\section{Resumo}

Chimenton, Alessandro Gaio; Rodriguez, Rafael Oswaldo Ruggiero Rodriguez. Fluxos geodésicos Finsler transitivos e aplicações. Rio de Janeiro, 2015. 82p. Tese de Doutorado - Departamento de Matemática, Pontifícia Universidade Católica do Rio de Janeiro.

Neste trabalho provamos que o fluxo geodésico de uma variedade Finsler de dimensão $n$ compacta, sem pontos conjugados e que é uma variedade de visibilidade uniforme é transitivo. Para isso, introduzimos versões Finsler dos conceitos de hiperbolicidade de Gromov e visibilidade de Eberlein e estudamos suas consequências. Como aplicação da transitividade, provamos que superfícies Finsler k-básicas compactas de gênero maior que um, sem pontos conjugados e com fibrados de Green contínuos são Riemannianas.

\section{Palavras-chave}

Visibilidade Finsler; Fluxos geodésicos transitivos; Grupos Gromovhiperbólicos; Superfícies Finsler k-básicas; 


\section{Abstract}

Chimenton, Alessandro Gaio; Rodriguez, Rafael Oswaldo Ruggiero Rodriguez (Advisor). Transitive Finsler geodesic flows and applications. Rio de Janeiro, 2015. 82p. Doctoral Thesis - Departamento de Matemática, Pontifícia Universidade Católica do Rio de Janeiro.

In this work we prove that the geodesic flow of a compact, $n$-dimensional Finsler manifold without conjugate points and which is an uniform visibility manifold is transitive. For this, we introduce Finsler versions of Gromov's hyperbolicity and Eberlein's visibility concepts and study its consequences. As an application of the transitivity, we prove that compact, k-basic Finsler surfaces without conjugate points, with genus greater than one and with continuous Green bundles are Riemannian.

\section{Keywords}

Finsler Visibility; Transitive geodesic flows; Gromov-hyperbolic groups; K-basic Finsler surfaces; 


\section{Sumário}

1 Geometria Finsler $\quad 15$

1.1 Variedades Finsler 15

$\begin{array}{ll}1.2 & \text { A conexão de Chern } \\ 1.3\end{array}$

1.3 Curvatura flag, campos de Jacobi e superfícies Finsler 21

2 Variedades Finsler de visibilidade $\quad 25$

2.1 Espaços métricos Finsler 25

2.2 Espaços Gromov-Finsler $\delta$-hiperbólicos $\quad 29$

2.3 Visiblidade em variedades Finsler 32

2.4 Bígonos geodésicos são estreitos 34

2.5 Visibilidade é equivalente à $\delta$-hiperbolicidade 39

2.6 Metrização de um grupo finitamente gerado 41

3 Visibilidade e a transitividade do fluxo geodésico $\quad 45$

3.1 As topologias de cones 46

3.2 Um homeomorfismo natural entre $\partial \tilde{M}(-\infty)$ e $\partial \tilde{M}(+\infty) \quad 49$

3.3 A ação de $\pi_{1}(M)$ em $\partial \tilde{M}(-\infty)$ e em $\partial \tilde{M}(+\infty)$

3.4 Geometria global de $\Gamma_{S}\left(\pi_{1}(M)\right) \quad 55$

3.5 Dualidade de Eberlein no contexto Finsler 58

3.6 Transitividade do fluxo geodésico $\quad 64$

4 Aplicações $\quad 68$

4.1 Campos de Jacobi assintóticos e divergência de raios geodésicos 68

4.2 O escalar de Cartan e os campos de Jacobi centrais 71

4.3 Fibrados de Green, operadores de Ricatti e campos de Jacobi centrais 73

4.4 Fibrados de Green e o escalar de Cartan 76

4.5 Transitividade e rigidez em superfícies Finsler k-básicas 78

$\begin{array}{lr}\text { Referências Bibliográficas } & 80\end{array}$ 


\section{Lista de Figuras}

2.1 Independentemente da escolha de $\ell_{i j}, \ell_{j k}, \ell_{k i}$, a aresta $\ell_{k i}$ está contida na "vizinhança $\delta$-tubular" de $\ell_{i j} \cup \ell_{j k}$

2.2 O bígono ligando $p$ e $q$

2.3 Bígonos arbitrariamente "largos" 36

2.4 A união das pseudo-bolas $\overline{B_{\rho_{n}(s)}^{-}(\gamma(s))}$ contém $\sigma_{n}((-\delta / C, \delta)) . \quad 37$

2.5 Imagem de $\overline{B_{\rho_{n}(s)}^{-}(\gamma(s))} \cap B_{\delta}^{+}\left(x_{n}\right)$ abaixo de um cone 38

3.1 Construindo a função $h$ em $\partial \tilde{M}(-\infty) . \quad 49$

3.2 Construindo a sequência $\gamma_{n}$. 54

3.3 Uma cópia "orientada" do grafo de Cayley em $\tilde{M}$. 56

3.4 Construindo a sequência $\tilde{v}_{n}$. 66 


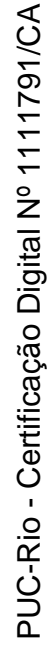




\section{Introdução}

A presente tese divide-se essencialmente em duas partes: na primeira (Capítulos 2 e 3), tratamos de problemas de classificação da dinâmica do fluxo geodésico para uma certa classe de variedades Finsler $n$-dimensionais. Uma métrica Finsler em uma variedade $M$ é uma família contínua $\{F(x, \cdot)\}_{x \in M}$ de normas não necessariamente simétricas em cada $T_{x} M$. Os exemplos mais imediatos de variedades Finsler são construídos tomando uma variedade Riemanniana $(M, g)$ e considerando $F(x, y)=\sqrt{g_{x}(y, y)}$, donde toda variedade Riemanniana é Finsler.

O estabelecimento de propriedades do fluxo geodésico de variedades Riemannianas, sob os mais variados conjuntos de hipóteses, é objeto de intensa investigação desde o início do século XX. A partir da década de 1920 foram obtidos diversos resultados em superfícies e variedades $n$-dimensionais Riemannianas de curvatura seccional negativa constante, particularmente no que concerne a existência de órbitas densas do fluxo geodésico. No contexto Finsler, sabe-se que variedades com curvatura flag negativa tem fluxo geodésico de tipo Anosov por um resultado de Foulon ([1] - 1992), o qual estende o caso Riemanniano.

No ambiente Riemanniano, Nielsen ([2] - 1925) foi o primeiro a obter geodésicas densas em superfícies hiperbólicas fechadas, orientáveis e de gênero maior que um. A hipótese da curvatura constante negativa foi bastante enfraquecida posteriormente por Koebe ([3] - trabalhos de 1927 a 1931), MorseHedlund ([4] - 1942) e Green ([5] - 1954). Mais recentemente, Eberlein ([6] 1972 e [7] - 1973) estudou a transitividade do fluxo geodésico em uma ampla classe de variedades Riemannianas sem pontos conjugados e com estrutura de visibilidade. Os métodos utilizados por Eberlein incluem uma detalhada descrição da ação do grupo de isometrias do recobrimento universal e, mais especificamente, a influência da existência de pontos fixos por alguma isometria no bordo do recobrimento, definido como o conjunto de classes assintóticas de geodésicas para $t>0$. Em particular, Eberlein prova a transitividade do fluxo geodésico introduzindo o conceito de dualidade. Nesta tese, estenderemos a noção de dualidade de Eberlein para variedades Finsler $n$-dimensionais de visibilidade uniforme e sem pontos conjugados, visando obter a transitividade 
do fluxo geodésico.

As propriedades do grupo fundamental de uma variedade compacta de visibilidade descritas por Eberlein são uma manifestação do que hoje se sabe sobre grupos Gromov $\delta$-hiperbólicos. Mais precisamente, em 1987 (posteriormente aos trabalhos de Eberlein, portanto), Gromov [8] publicou um estudo sistemático e extremamente abrangente sobre grupos finitamente gerados e seus grafos de Cayley munidos da métrica da palavra $^{1}$. Esta métrica faz do grafo de Cayley um espaço métrico geodésico sobre o qual o grupo subjacente age. Gromov, entre diversos resultados, prova propriedades dinâmicas dessa ação e em particular mostra que se o grupo é $\delta$-hiperbólico, sua ação no bordo ideal do grafo de Cayley (definido analogamente como o conjunto de classes de geodésicas métricas assintóticas para $t>0$ ) é topologicamente transitiva e tem alguma órbita densa. É possível verificar que o grupo de isometrias de uma variedade Riemanniana compacta de visibilidade uniforme e sem pontos conjugados é $\delta$-hiperbólico. Ocorre que o grupo fundamental de uma tal variedade é quase-isométrico ao recobrimento universal da mesma, devido ao Lema de Švarc-Milnor [14]. A quase-isometria transfere propriedades dinâmicas da ação do grupo de isometrias no grafo de Cayley para a ação do grupo fundamental no bordo da variedade. De fato, mostraremos neste trabalho, para uma variedade Finsler $M$ compacta, sem pontos conjugados e de visibilidade uniforme, que a existência de uma órbita densa da ação de $\pi_{1}(M)$ no bordo de seu grafo de Cayley implica a existência de uma órbita densa do fluxo geodésico. Para isso, introduziremos noções de $\delta$-hiperbolicidade e visibilidade adequadas ao ambiente Finsler, já que não dispomos exatamente de um espaço métrico (como são as variedades Riemannianas), pois uma métrica Finsler não é necessariamente simétrica nas fibras tangentes. Com isso, veremos que a potencial falta de simetria das métricas Finsler não impõe restrições para a obtenção da generalização natural dos resultados sobre variedades Riemannianas de visibilidade estabelecidos, na década de 1970, por Eberlein.

Na segunda parte desta tese (Capítulo 4), estudamos problemas de classificação de superfícies Finsler impondo condições sobre sua curvatura e topologia utilizando os resultados da primeira parte. Como mencionado no início da introdução, toda variedade Riemanniana é naturalmente dotada de uma métrica Finsler. Uma questão natural que emerge é, portanto, a seguinte:

\footnotetext{
${ }^{1}$ De fato, desde seu trabalho, toda uma área de pesquisa tem se expandido enormemente originando o que convencionou-se chamar teoria geométrica de grupos - ver por exemplo [9], [10], [11], [12] e o excelente texto de P.de La Harpe [13].
} 
Que hipóteses sobre a métrica Finsler F e/ou sobre a topologia de $M$ implicam que

$$
F(x, y)=\sqrt{g_{x}(y, y)}
$$

para alguma métrica Riemanniana $g$ em $M$ ?

Respostas para esta pergunta têm sido obtidas sob diferentes hipóteses. O célebre Teorema de Akbar-Zadeh [15] estabelece que toda superfície compacta de curvatura flag $K \equiv-1$ é Riemanniana. A demonstração usa as identidades de Bianchi para métricas Finsler, que enunciamos brevemente no final do Capítulo 1. Akbar-Zadeh prova no mesmo artigo que a prescrição de curvatura flag $K \equiv 0$ implica que a superfície subjacente é um toro ou uma garrafa de Klein, mas não necessariamente que a métrica Finsler é Riemanniana. A hipótese $K \equiv 1$ parece ser ainda menos restritiva. Bryant [16] mostrou ser possível construir em $\mathbb{S}^{2}$ toda uma família de métricas Finsler não-Riemannianas satisfazendo a essa condição sobre a curvatura. De fato, o autor prova que se $K \equiv 1$, então $I^{2}+J^{2}$ é constante ao longo de geodésicas. Aqui, as quantidades $I$ e $J$ são respectivamente o escalar de Cartan e o curvatura de Landsberg, oriundas das equações estruturais das superfícies Finsler, enunciadas também no final do Capítulo 1. Bryant mostra ainda que a hipótese da compacidade do Teorema de Akbar-Zadeh é necessária.

O enfraquecimento da hipótese sobre a curvatura flag tornam, naturalmente, ainda mais complexa a classificação de superfícies Finsler como Riemannianas. Por exemplo, em um trabalho de 2005, Z.Shen mostra que variedades Finsler $n$-dimensionais sem bordo, satisfazendo $K<0$ e com $S$-curvatura constante são Riemannianas [17]. Paternain [18] mostrou que superfícies Finsler com estrutura de Landsberg analítica ou k-básicas de curvatura $K \leqslant 0$ e gênero maior que 1 são Riemannianas. Mais recentemente, Ruggiero e Barbosa [19] provaram que superfícies Finsler compactas $C^{4}$, de tipo Landsberg, sem pontos conjugados e de gênero maior que 1 são Riemannianas, usando quantidades conservadas pelo fluxo geodésico.

É natural relaxar a condição $K \leqslant 0$ impondo apenas a ausência de pontos conjugados. No Capítulo 4 desta tese, apresentaremos uma contribuição à teoria de classificação mostrando que superfícies Finlser sem pontos conjugados, k-básicas, compactas, de gênero maior que um e com fibrados de Green contínuos são Riemannianas. A demonstração do resultado usa a transitividade do fluxo geodésico de uma tal superfície (obtida como consequência dos resultados dos Capítulos 2 e 3), aliada com propriedades do escalar de Cartan sobre órbitas deste fluxo e também de certos campos de Jacobi assintóticos, obtidos como limites de campos de Jacobi com um zero. A continuidade dos 
fibrados de Green e a transitividade do fluxo geodésico nos permitem globalizar condições locais sobre o escalar de Cartan e, em particular, mostrar que ele se anula em um conjunto denso do fibrado tangente da superfície - e portanto se anula identicamente. O Teorema de Deicke (Teorema 1.10) mostra então que a superfície em questão é Riemanniana. Mas os métodos empregados na demonstração são bastante dependentes da bidimensionalidade e da continuidade desses fibrados. A exclusão desta última hipótese, por exemplo, tem sido objeto de estudos correntes e ainda não publicados de R.Ruggiero e P.Foulon. 


\section{Geometria Finsler}

Neste capítulo, apresentaremos alguns resultados básicos da teoria das variedades Finsler que serão usados ao longo da presente tese. Nossas referências são o texto clássico de Bao, Chern e Shen [20], além de [21], [22]. Usaremos as notações

$$
T M-\{0\}:=\{(x, y) \in T M / y \neq 0\}
$$

e

$$
T^{*} M-\{0\}:=\left\{(x, y) \in T^{*} M / y \neq 0\right\} .
$$

Sejam $\pi: T M \longrightarrow M$ a projeção natural do fibrado tangente sobre $M$. Consideraremos os seguintes fibrados vetoriais sobre $T M-\{0\}$ :

$$
\pi^{*} T M:=\bigcup_{(x, y) \in T M-\{0\}} T_{x} M \quad \text { e } \quad \pi^{*} T^{*} M:=\bigcup_{(x, y) \in T M-\{0\}} T_{x}^{*} M .
$$

Daqui em diante, dado $p \in M$ e $U$ uma vizinhança parametrizada de $M$, digamos por $\mathbf{x}: U \longrightarrow \mathbb{R}^{n}, \mathbf{x}(q)=\left(x_{1}(q), \ldots, x_{n}(q)\right)$ para $q \in U$, consideraremos os referenciais locais de $T M$ e de $T^{*} M$ como sendo $\left\{\frac{\partial}{\partial x_{i}}(q)\right\}$ e $\left\{d x_{i}(q)\right\}$, os quais fornecem as cartas locais usuais de $T M$ e de $T^{*} M$ que associam $y^{i} \frac{\partial}{\partial x_{i}}(q)$ e $y^{i} d x_{i}(q)$ a $\left(x_{1}(q), \ldots, x_{n}(q), y^{1}, \ldots, y^{n}\right)$. Neste sistema de coordenadas, as derivadas parciais de uma função $f: T M \longrightarrow \mathbb{R}$ serão denotadas por $f_{x_{i}}$ ou $f_{y^{i}}$ (analogamente para funções $f: T^{*} M \longrightarrow \mathbb{R}$ ).

\section{1}

\section{Variedades Finsler}

Definição 1.1. Uma métrica Finsler em uma variedade diferenciável ndimensional $M$ é uma função $F: T M \longrightarrow \mathbb{R}$ satisfazendo os seguintes axiomas:

1. F é de classe $C^{\infty}$ em $T M-\{0\}$ e contínua em $T M$.

2. $F(x, \lambda y)=\lambda F(x, y)$ para todo $\lambda>0$ e para todo $(x, y) \in T M$. 
3. Para cada $(x, y) \in T M-\{0\}$, a forma bilinear em $T_{p} M$ dada por

$$
g_{(x, y)}(u, v):=\frac{1}{2} \frac{\partial^{2}}{\partial s \partial t}\left[F^{2}(x, y+s u+t v)\right]_{s=t=0}=\left(g_{i j}\right)_{(x, y)} u^{i} v^{j}
$$

onde $\left(g_{i j}\right)_{(x, y)}=\frac{1}{2} \frac{\partial^{2} F^{2}}{\partial y^{i} \partial y^{j}}(x, y)$ e $u, v \in T_{x} M$, é um produto interno. A família $\left\{g_{(x, y)}\right\}_{(x, y) \in T M-\{0\}}$ é chamada de tensor fundamental.

$O \operatorname{par}(M, F)$ é denominado variedade Finsler.

Observe que $g_{(x, y)}=g_{(x, \lambda y)}$ e que $g_{(x, y)}$ independe de $y$ se e somente se $g_{(x, y)}$ é Riemanniana. É natural portanto medir a variação de $g_{(x, y)}$ nas demais direções tangentes. Definimos então

$$
\begin{aligned}
A_{(x, y)}(u, v, w) & :=\frac{F(x, y)}{2} \frac{d}{d r}\left[g_{(x, y+r w)}(u, v)\right]_{r=0} \\
& =\frac{F}{4} \frac{\partial^{3}}{\partial r \partial s \partial t}\left[F^{2}(x, y+s u+t v+r w)\right]_{\mid r=s=t=0}
\end{aligned}
$$

A família $A:=\left\{A_{(x, y)}\right\}_{y \in T_{x} M-\{0\}}$ é o tensor de Cartan. Quando não houver risco de ambiguidade, escreveremos apenas $g_{y}$ e $A_{y}$ em vez de $g_{(x, y)}$ e $A_{(x, y)}$ respectivamente.

Considerando em cada $T_{x}^{*} M$ o referencial $\left\{d x_{i}\right\}$, dual de $\left\{\frac{\partial}{\partial x_{i}}\right\}$, a aplicação $\pi: \pi^{*} T M \longrightarrow T M-\{0\}$ permite, com um abuso de notação, definirmos o referencial de 1 -formas $d x_{i}:=\pi^{*} d x_{i}$ em $\pi^{*} T M$. Neste referencial, o tensor fundamental e o tensor de Cartan se escrevem respectivamente como

$$
g=g_{i j} d x_{i} \otimes d x_{j} \quad \text { e } \quad A=A_{i j k} d x_{i} \otimes d x_{j} \otimes d x_{k}
$$

onde $A_{i j k}=\frac{F}{4}\left[F^{2}\right]_{y^{i} y^{j} y^{k}}$. Temos que $A$ é uma forma trilinear simétrica em cada fibra de $\pi^{*} T M$ e satisfaz a condição de homogeneidade $A_{(x, \lambda y)}(u, v, w)=$ $A_{(x, y)}(u, v, w)$ para $\lambda>0$. Além disso, $A=0$ se e somente se $F=\sqrt{\mathfrak{g}}$ onde $\mathfrak{g}$ é uma métrica Riemanniana em $M$. Em outras palavras, a família $A$ mede o quão "não-Riemanniana" é uma métrica Finsler.

O comprimento de uma curva diferenciável por partes $\gamma:[a, b] \longrightarrow M$ é o número real

$$
L_{F}(\gamma):=\int_{a}^{b} F\left(\gamma(t), \gamma^{\prime}(t)\right) d t
$$

A distância entre $p, q \in M$ é o número real $d_{F}(p, q):=\inf _{\gamma \in \Gamma} L_{F}(\gamma)$, onde $\Gamma$ é o conjunto de todas as curvas $\sigma:[a, b] \longrightarrow M$ diferenciáveis por partes tais que $\sigma(a)=p$ e $\sigma(b)=q$. Observamos que em geral $d_{F}(p, q) \neq d_{F}(q, p)$. Entretanto, se $M$ é compacta, existe $C>0$ tal que $d_{F}(p, q) \leqslant C d_{F}(q, p)$ para todo $p, q \in M$. Além disso, $d_{F}(p, q)=0$ se e só se $p=q$ e vale a desigualdade 
triangular: para todo $p, q, r \in M$,

$$
d_{F}(p, q) \leqslant d_{F}(p, r)+d_{F}(r, q)
$$

Uma curva $\gamma$ diferenciável tem velocidade constante $c$ se $F\left(\gamma(t), \gamma^{\prime}(t)\right)=c$ para todo $t$, é unitária se tem velocidade constante 1 e é minimizante se $L_{F}\left(\gamma_{\mid[s, t]}\right)=d_{F}(\gamma(s), \gamma(t))$ para todo $s<t$. Uma isometria Finsler, ou simplesmente isometria, é um difeomorfirsmo $\phi: M \longrightarrow M$ tal que $F(p, v)=$ $F\left(\phi(p),(d \phi)_{p}(v)\right)$ para todo $(p, v) \in T M$. É claro que uma isometria preserva comprimento de curvas e, portanto, distâncias.

A energia de uma curva $\gamma:[a, b] \longrightarrow M$ é o número real

$$
\mathcal{E}(\gamma):=\frac{1}{2} \int_{a}^{b} F^{2}\left(\gamma(t), \gamma^{\prime}(t)\right) d t
$$

e uma curva $\gamma:[a, b] \longrightarrow M$ que minimiza $\mathcal{E}$ dentre todas as curvas orientadas que ligam $\gamma(a)$ a $\gamma(b)$ satisfaz $d_{F}(\gamma(a), \gamma(b))=L_{F}(\gamma)$. Uma geodésica ligando $\gamma(a)$ e $\gamma(b)$ é uma solução das equações de Euler-Lagrange para o funcional $\mathcal{E}$ acima:

$$
\frac{d}{d t}\left(F^{2}\right)_{y^{i}}-\left(F^{2}\right)_{x_{i}}=0 .
$$

O fluxo geodésico é $(M, F)$ é simplesmente o fluxo de Euler-Lagrange do sistema de equações acima.

Dada uma curva diferenciável por partes $\gamma:[a, b] \longrightarrow M$, denotaremos por $\gamma^{-1}:[a, b] \longrightarrow M$ a curva definida por

$$
\gamma^{-1}(t)=\gamma(b-t+a)
$$

que é simplesmente uma reparametrização afim de $\gamma$ no sentido oposto ao seu parâmetro original. Em geral temos $L(\gamma) \neq L\left(\gamma^{-1}\right)$. Além disso, como a métrica Finsler não é necessariamente simétrica nas fibras tangentes, mesmo admitindo que $\gamma$ é uma geodésica, $\gamma^{-1}$ pode não o ser.

Daqui até o fim do trabalho, $M$ será uma variedade compacta de dimensão $n$ e $\tilde{M}$ denotará seu recobrimento universal. O fibrado tangente unitário de $M$ é o conjunto

$$
\mathcal{S} M:=\{(x, y) \in T M / F(x, y)=1\}
$$

que é uma variedade de dimensão $2 n-1$, também descrita como a reunião das indicatrizes

$$
\mathcal{S}_{x}:=\left\{y \in T_{x} M / F(x, y)=1\right\}
$$


para $x \in M$, que são hiperfícies fechadas convexas em cada $T_{x} M$. Durante todo o texto, usaremos a constante

$$
C:=\max _{(p, v) \in \mathcal{S} M}\{F(p, v), F(p,-v)\}=\max _{(p, v) \in \mathcal{S} \tilde{M}}\{\tilde{F}(p, v), \tilde{F}(p,-v)\} .
$$

Note que $C \geqslant 1$.

Lema 1.2. Sejam $(M, F)$ uma variedade Finsler compacta e $\gamma:[a, b] \longrightarrow \tilde{M}$ uma curva diferenciável por partes com $F\left(\gamma(t), \gamma^{\prime}(t)\right)=1$ para todo $t \in[a, b]$ Então

$$
L_{\tilde{F}}\left(\gamma^{-1}\right) \leqslant C L_{\tilde{F}}(\gamma)
$$

Demonstração. Considere a curva $\gamma^{-1}:[a, b] \longrightarrow \tilde{M}$. Então $\gamma^{-1}(a)=\gamma(b)=y$ e $\gamma^{-1}(b)=\gamma(a)=x$. Além disso,

$$
\begin{aligned}
L_{\tilde{F}}\left(\gamma^{-1}\right) & =\int_{a}^{b} \tilde{F}\left(\gamma^{-1}(t),\left(\gamma^{-1}\right)^{\prime}(b-t+a)\right) d t \\
& =\int_{a}^{b} \tilde{F}\left(\gamma(b-t+a),-\gamma^{\prime}(b-t+a)\right) d t \\
& \leqslant C \int_{a}^{b} d t=C L_{\tilde{F}}(\gamma),
\end{aligned}
$$

pois $\left(\gamma(b-t+a), \gamma^{\prime}(b-t+a)\right) \in \mathcal{S} \tilde{M}$ para todo $t \in[a, b]$.

Corolário 1.3. Se $\gamma:[a, b] \longrightarrow \tilde{M}$ é uma curva não necessariamente unitária, então

$$
L_{\tilde{F}}\left(\gamma^{-1}\right) \leqslant C L_{\tilde{F}}(\gamma) .
$$

Demonstração. Seja $\phi:[c, d] \longrightarrow[a, b] \operatorname{com} \phi^{\prime}(s)>0, \phi(c)=a$ e $\phi(d)=b$. Pelo teorema de mudança de variáveis,

$$
\begin{aligned}
L_{\tilde{F}}(\bar{\gamma}) & =\int_{c}^{d} \tilde{F}\left(\bar{\gamma}(s), \bar{\gamma}^{\prime}(s)\right) d s \\
& =\int_{c}^{d} \tilde{F}\left(\gamma(\phi(s)), \phi^{\prime}(s) \gamma^{\prime}(\phi(s))\right) d s \\
& =\int_{c}^{d} \tilde{F}\left(\gamma(\phi(s)), \gamma^{\prime}(\phi(s))\right) \cdot \phi^{\prime}(s) d s \\
& =\int_{a}^{b} \tilde{F}\left(\gamma(t), \gamma^{\prime}(t)\right) d t=L_{\tilde{F}}(\gamma),
\end{aligned}
$$

pois $\tilde{F}(p, \lambda v)=\lambda \tilde{F}(p, v)$ para todo $\lambda>0$. Portanto, reparametrizações que preservam orientação não mudam comprimentos de curvas. Denotando por $\bar{\gamma}$ a reparametrização unitária de $\gamma$, temos que $\bar{\gamma}^{-1}(s)=\gamma^{-1}(\phi(s))$. Assim,

$$
L_{\tilde{F}}\left(\gamma^{-1}\right)=L_{\tilde{F}}\left(\bar{\gamma}^{-1}\right) \leqslant C L_{\tilde{F}}(\bar{\gamma})=C L_{\tilde{F}}(\gamma)
$$


como queríamos provar.

Corolário 1.4. Sejam $(M, F)$ variedade Finsler compacta, $d_{\tilde{F}}$ a distância Finsler induzida por $L_{\tilde{F}}$ em $\tilde{M}$. Então, para todo $x, y \in \tilde{M}$ vale

$$
d_{\tilde{F}}(y, x) \leqslant C d_{\tilde{F}}(x, y) .
$$

Demonstração. Basta tomar uma geodésica minimizante $\gamma$ ligando $x, y$ e aplicar o lema anterior.

\section{2}

\section{A conexão de Chern}

Sejam $(M, F)$ uma variedade Finsler e $\Gamma(T M)$ o conjunto de campos de vetores diferenciáveis em $M$. Uma conexão afim em $T M$ é um aplicação bilinear $\nabla: \Gamma(T M) \times \Gamma(T M) \longrightarrow \Gamma(T M)$ que associa $(X, Y)$ a $\nabla_{X} Y$ e satisfaz a regra de Leibniz

$$
\nabla_{X}(f Y)=X(f) Y+f \nabla_{X} Y
$$

para toda $f \in C^{\infty}(M)$ e para todo $X, Y \in \Gamma(T M)$. O seguinte importante resultado é devido a Chern.

Teorema 1.5 (Chern). Seja $(M, F)$ uma variedade Finsler. Existe uma aplicação

$$
\begin{aligned}
\nabla: \Gamma(T M-\{0\}) \times \Gamma(T M) \times \Gamma(T M) & \longrightarrow \Gamma(T M) \\
(V, X, Y) & \longmapsto \nabla_{X}^{V} Y
\end{aligned}
$$

satisfazendo as seguintes condições:

1. Para todo $V \in \Gamma(T M-\{0\})$, a aplicação $\nabla^{V}$ é uma conexão afim.

2. $\nabla^{V}$ é livre de torção, ou seja,

$$
\nabla_{X}^{V} Y-\nabla_{Y}^{V} X=[X, Y]
$$

3. $\nabla^{V}$ é quase-métrica, ou seja,

$$
X\left(g_{V}(Y, Z)\right)=g_{V}\left(\nabla_{X}^{V} Y, Z\right)+g_{V}\left(Y, \nabla_{X}^{V} Z\right)+2 C_{V}\left(\nabla_{X}^{V}, Y, Z\right),
$$

onde $C=\frac{1}{F} A$. 
Além disso, vale a fórmula de Koszul generalizada: para todo $X, Y, Z \in \Gamma(T M)$,

$$
\begin{aligned}
2 g_{V}\left(\nabla_{X}^{V} Y, Z\right) & =X\left(g_{V}(Y, Z)\right)+Y\left(g_{V}(Z, X)\right)-Z\left(g_{V}(X, Y)\right) \\
& +g_{V}([X, Y], Z)-g_{V}([Y, Z], X)+g_{V}([Z, X], Y) \\
& -2\left[C_{V}\left(\nabla_{X}^{V} V, Y, Z\right)+C_{V}\left(\nabla_{Y}^{V} V, Z, X\right)-C_{V}\left(\nabla_{Z}^{V}, X, Y\right)\right]
\end{aligned}
$$

e esta fórmula determina unicamente $V$. A aplicação $\nabla$ é chamada conexão de Chern.

Fixado $V \in \Gamma(T M-\{0\})$ e usando a conexão de Chern, podemos definir "símbolos de Christoffel" em $T M-\{0\}$ localmente via

$$
\nabla_{\frac{\partial}{\partial x_{i}}}^{V} \frac{\partial}{\partial x_{j}}(x)=\Gamma_{i j}^{k}\left(x, V_{x}\right) \frac{\partial}{\partial x_{k}}
$$

e com estes, definimos (localmente) a derivada covariante de um campo de vetores $X(t)=X_{i} \frac{\partial}{\partial x_{i}}$ ao longo de uma curva $\gamma(t)$ por

$$
D_{\gamma^{\prime}} X(\gamma(t)):=\left.\left(\frac{d X_{k}}{d t}+\Gamma_{i j}^{k}\left(\gamma(t), \gamma^{\prime}(t)\right) \gamma_{i}^{\prime} X_{j}\right) \frac{\partial}{\partial x_{k}}\right|_{\gamma(t)}
$$

Com essa linguagem, as soluções das equações de Euler-Lagrange (1-2) são exatamente as soluções da equação das geodésicas

$$
D_{\gamma^{\prime}} \gamma^{\prime}=0
$$

e tais soluções são geodésicas de velocidade constante. Uma métrica Finsler é positivamente completa (respectivamente, completa) se toda geodésica em $[a, b]$ pode ser estendida para $[a, \infty)$ (respectivamente, para $(-\infty, \infty))$.

Seja $(M, F)$ uma variedade Finsler positivamente completa. Para cada $x \in M$, definimos a aplicação exponencial $\exp _{x}: T_{x} M \longrightarrow M$ por

$$
\exp _{x}(y):=\gamma(1)
$$

onde $\gamma:[0, \infty) \longrightarrow M$ é a única geodésica tal que $\gamma(0)=x$ e $\gamma^{\prime}(0)=y$.

Teorema 1.6 (Hopf-Rinow - [21], p.49, Theorem 1.6.9). Seja $(M, F)$ uma variedade Finsler. Então as seguintes afirmações são equivalentes:

1. $(M, F)$ é positivamente completa.

2. existe $x \in M$ tal que $\exp _{x}$ está definida em $T_{x} M$.

3. para todo $x \in M$, $\exp _{x}$ está definida em $T_{x} M$. 
Qualquer uma das afirmações acima implica a seguinte: para quaisquer $p, q \in$ $M$ existe uma geodésica $\gamma$ ligando $p$ a q tal que $L_{F}(\gamma)=d_{F}(p, q)$.

No contexto desta tese, trataremos de variedades Finsler compactas, as quais são sempre completas e positivamente completas. Como consequência do exposto acima e do Teorema de Hopf-Rinow para variedades Finsler, temos a seguinte:

Proposição 1.7. Seja $(M, F)$ uma variedade Finsler compacta. Então $\left(\tilde{M}, d_{\tilde{F}}\right)$ é um espaço métrico Finsler completo e geodésico.

\section{3}

\section{Curvatura flag, campos de Jacobi e superfícies Finsler}

Para cada campo $V$ sem zeros definido em um aberto $U \subset M$, considere a conexão afim $\nabla^{V}$ associada. É possível provar (ver por exemplo [23], Proposition 3.3) que dado $p \in U$, a quantidade

$$
R^{V}(X, Y, Z):=\nabla_{X}^{V} \nabla_{Y}^{V} Z-\nabla_{Y}^{V} \nabla_{X}^{V} Z-\nabla_{[X, Y]}^{V} Z
$$

depende apenas dos valores de $V$ ao longo da única curva integral de $V$ que passa por $p$. A equação acima define localmente o tensor de curvatura da métrica Finsler $F$ com campo de referência $V$. Um flag em $T_{x} M$ é um par $(V, \pi)$ onde $V=\gamma^{\prime}(0) \in T_{x} M$, com $\gamma$ a única geodésica unitária com condições iniciais $p$ e $V$ e $\pi \subset T_{x} M$ é um plano tal que $\pi=\operatorname{span}\{V, U\}$ para algum $U \in T_{x} M$ (aqui o campo de referência é qualquer extensão local do campo $\left.\gamma^{\prime}(t)\right)$. O vetor $V$ é chamado pólo do flag $(V, \pi)$. A curvatura flag de $(M, F)$ em $(V, \pi)$ é definida por

$$
K(V, \pi):=\frac{g_{V}\left(R^{V}(U, V) V, U\right)}{g_{V}(V, V) g_{V}(U, U)-g_{V}(V, U)} .
$$

Definição 1.8. Em uma variedade Finsler $(M, F)$, um campo de vetores $J(t)$ ao longo de uma geodésica $\gamma:[a, b] \longrightarrow M$ é um campo de Jacobi se

$$
D_{\gamma^{\prime}} D_{\gamma^{\prime}} J+R^{\gamma^{\prime}} J=0
$$

para todo $t \in[a, b]$, onde $R^{\gamma^{\prime}}$ denota a curvatura flag de $M$ em $\left(\gamma^{\prime}, \pi\right)$, com $\pi$ sendo o plano gerado por $\gamma^{\prime}$ e J. O ponto $\gamma(b)$ é conjugado ao ponto $\gamma(a)$ ao longo de $\gamma$ se existe algum campo de Jacobi $J$ ao longo de $\gamma$, não nulo, tal que $J(a)=0$ e $J(b)=0$.

Campos de Jacobi são obtidos derivando variações próprias $C^{2}$ de uma geodésica por geodésicas, como no caso Riemanniano. Diversas propriedades 
dos campos de Jacobi Riemannianos são reproduzidas no contexto Finsler, como as que enunciamos abaixo:

Proposição 1.9. Seja $\gamma(t)=\exp _{x}(t v), t \in[0, r]$, uma geodésica unitária. Então as seguintes afirmações são equivalentes:

1. O ponto $q=\gamma(r)$ não é conjugado a $p=\gamma(0)$ ao longo de $\gamma$.

2. Qualquer campo de Jacobi ao longo de $\gamma$ que se anula em $p$ e qé identicamente nulo.

3. Dado $V \in T_{p} M$ e $W \in T_{q} M$, existe um único campo de Jacobi $J:[0, r] \in T M$ ao longo de $\gamma$ tal que $J(0)=V$ e $J(r)=W$.

4. A aplicação linear $d\left(\exp _{p}\right)_{\text {tv }}$ é não singular.

Além disso, se $(M, F)$ não tem pontos conjugados, o recobrimento universal $(\tilde{M}, \tilde{F})$ com a estrutura $\tilde{F}$, pull-back de $F$ pela projeção natural $\pi: \tilde{M} \longrightarrow M$, é difeomorfo a $\mathbb{R}^{n}$ e toda geodésica unitária $\tilde{\gamma}: \mathbb{R} \longrightarrow \tilde{M}$ é minimizante.

Seja $(M, F)$ uma superfície Finsler compacta. As quantidades $g$ e $A$ são homogêneas de grau zero em $y$ e portanto podemos pensá-las como quantidades definidas em $\mathcal{S} M$. Note que o campo de vetores em $\mathcal{S} M$ dado por

$$
\ell_{(x, y)}:=\frac{y^{1}}{F(y)} \frac{\partial}{\partial x_{1}}+\frac{y^{2}}{F(y)} \frac{\partial}{\partial x_{2}}
$$

tem as propriedades

$$
g_{y}(\ell, \ell)=1 \quad \text { e } \quad g_{y}\left(y(t), \ell_{(y(t))}\right)=0
$$

para qualquer parametrização unitária da indicatriz. Em outras palavras, a seção $\ell$ de $\pi^{*} T M$ é $g_{y}$-ortogonal a $\mathcal{S}_{x}$. Ao mesmo tempo, campo de vetores

$$
e_{1(x, y)}:=\frac{1}{\sqrt{g_{y}}}\left(\frac{\partial F}{\partial y^{2}} \frac{\partial}{\partial x_{1}}-\frac{\partial F}{\partial y^{1}} \frac{\partial}{\partial x_{2}}\right)
$$

onde $\sqrt{g_{y}}=\sqrt{\operatorname{det}\left(g_{i j}\right)(y)}$, satisfaz $g_{y}\left(e_{1}, e_{1}\right)=1$ e $g_{y}\left(e_{1}, \ell\right)=0$. Denotando $\ell=e_{2}$, temos que o referencial de Berwald $\left\{e_{1}, e_{2}\right\}$ é $g$-ortonormal e está definido globalmente em $\mathcal{S} M$. Sejam $\left\{\omega^{1}, \omega^{2}\right\}$ as 1 -formas duais a $\left\{e_{1}, e_{2}\right\}$, a saber

$$
\begin{aligned}
\omega^{1} & =\frac{\sqrt{g_{y}}}{F}\left(y^{2} d x_{1}-y^{1} d x_{2}\right) \\
\omega^{2} & =\frac{\partial F}{\partial y^{1}} d x_{1}+\frac{\partial F}{\partial y^{2}} d x_{2} .
\end{aligned}
$$


e

$$
\omega^{3}:=\frac{\sqrt{g_{y}}}{F}\left(y^{2} \frac{\delta y^{1}}{F}-y^{1} \frac{\delta y^{2}}{F}\right) .
$$

Aqui, $\delta y^{i}:=d y^{i}+N_{j}^{i} d x_{i}$, onde $N_{j}^{i}$ são os coeficientes locais da conexão nãalinear Finsleriana (para detalhes, ver seção 2.3 de [20]). O referencial de 1formas $\left\{\omega^{1}, \omega^{2}, \omega^{3}\right\}$ está globalmente definido em $\mathcal{S} M$ e seu referencial dual é

$$
\begin{aligned}
\hat{e}_{1} & =\frac{1}{\sqrt{g_{y}}}\left(\frac{\partial F}{\partial y^{2}} \frac{\delta}{\delta x_{1}}-\frac{\partial F}{\partial y^{1}} \frac{\delta}{\delta x_{2}}\right) \\
\hat{e}_{2} & =\frac{y^{1}}{F} \frac{\delta}{\delta x_{1}}+\frac{y^{2}}{F} \frac{\delta}{\delta x_{2}} \\
\hat{e}_{3} & =\frac{F}{\sqrt{g_{y}}}\left(\frac{\partial F}{\partial y^{2}} \frac{\partial}{\partial y^{1}}-\frac{\partial F}{\partial y^{1}} \frac{\partial}{\partial y^{2}}\right) .
\end{aligned}
$$

Aqui, $\frac{\delta}{\delta x_{i}}:=\frac{\partial}{\partial x_{i}}-N_{i}^{j} \frac{\partial}{\partial y^{i}}$. Em particular, $\hat{e}_{3}$ é tangente às indicatrizes e $\hat{e}_{2}$ é tangente ao fluxo geodésico. Usando as 1-formas $\left\{\omega^{1}, \omega^{2}, \omega^{3}\right\}$, podemos definir uma estrutura Riemanniana em $\mathcal{S} M$, chamada métrica de tipo Sasaki, dada por

$$
\omega^{1} \otimes \omega^{1}+\omega^{2} \otimes \omega^{2}+\omega^{3} \otimes \omega^{3}
$$

em relação à qual o referencial $\left\{\hat{e}_{1}, \hat{e}_{2}, \hat{e}_{3}\right\}$ é ortonormal. Tomando a derivada exterior do referencial $\left\{\omega^{1}, \omega^{2}, \omega^{3}\right\}$, obtemos as equações estruturais de uma superfície Finsler:

$$
\begin{aligned}
d \omega^{1} & =-I \omega^{1} \wedge \omega^{3}+\omega^{2} \wedge \omega^{3} \\
d \omega^{2} & =-\omega^{1} \wedge \omega^{3} \\
d \omega^{3} & =K \omega^{1} \wedge \omega^{2}-J \omega^{1} \wedge \omega^{3} .
\end{aligned}
$$

As funções $I, J, K$ estão definidas em $\mathcal{S} M$ e são respectivamente o escalar de Cartan, o escalar de Landsberg e a curvatura Gaussiana. De fato, $K$ é a curvatura flag calculada em $(x, y) \in \mathcal{S} M$ com respeito ao flag $\left(y, T_{x} M\right)$. Podese provar que

$$
I=A_{111}=A\left(e_{1}, e_{1}, e_{1}\right) .
$$

Enunciamos também as identidades de Bianchi: tomando derivadas exteriores nas equações estruturais, temos

$$
\begin{aligned}
J & =I_{2}=\frac{1}{F}\left(y^{1} \frac{\delta I}{\delta x_{1}}+y^{2} \frac{\delta I}{\delta x_{2}}\right) \\
K_{3}+K I+J_{2} & =0 .
\end{aligned}
$$

Aqui, os sub-índices indicam derivadas nas direções $\hat{e}_{1}, \hat{e}_{2}, \hat{e}_{3}$. 
A demonstração do Teorema A desta tese (ver Capítulo 4) usará o seguinte resultado, que caracteriza dentre as superfícies Finsler aquelas que são Riemannianas.

Teorema 1.10 (Deicke). Uma superfície Finsler $(M, F)$ é Riemanniana se e somente se $I(\eta)=0$ para todo $\eta \in \mathcal{S} M$.

Por fim, o Teorema A mencionado será enunciado no Capítulo 4 dentro da seguinte classe de superfícies Finsler.

Definição 1.11. Uma superfície Finsler $(M, F)$ é k-básica se sua curvatura Gaussiana $K$ é uma função apenas dos pontos de $M$. 


\section{2}

\section{Variedades Finsler de visibilidade}

Este capítulo contém resultados centrais para o estudo da transitividade do fluxo geodésico em variedades Finsler de visibilidade, compactas e sem pontos conjugados. Sua organização é a seguinte: primeiramente (seções $2.1 \mathrm{e}$ 2.2), definiremos um conceito de hiperbolicidade para espaços métricos Finsler, estendendo a noção de hiperbolicidade de espaços métricos introduzida por Gromov em [8] e intensamente estudada desde então. Em seguida (seção 2.3), estendemos também a noção de visibilidade, introduzida para variedades Riemannianas sem pontos conjugados por Eberlein-O'Neill em [7], para o contexto das variedades Finsler compactas e sem pontos conjugados.

Em variedades Riemannianas sem pontos conjugados nas quais os raios geodésicos com ponto em comum divergem uniformemente, sabe-se desde [24] que as noções de $\delta$-hiperbolicidade de Gromov e visibilidade de Eberlein são equivalentes. Assim, o principal objetivo deste capítulo é mostrar que os análogos Finsler dos conceitos de visibilidade e hiperbolicidade por nós aqui definidos são também equivalentes, supondo divergência de raios geodésicos (seção 2.5). Para provar isso, usaremos fortemente um controle global da distância de geodésicas que ligam dois pontos quaisquer da variedade Finsler, independentemente de suas orientações (seção 2.4). Este controle será obtido como consequência de propriedades das soluções das equações de Ricatti em variedades Finsler sem pontos conjugados.

Por último, recordaremos brevemente a noção de métrica da palavra no grafo de Cayley de um grupo finitamente gerado (seção 2.6). Em particular, construiremos uma compactificação para o grafo de Cayley de um grupo hiperbólico. O objetivo é descrever a ação do grupo em seu grafo de Cayley, visando enunciar alguns importantes resultados devidos a Gromov incluídos em [8], os quais serão úteis no Capítulo 3.

\section{1}

\section{Espaços métricos Finsler}

Começamos com algumas noções básicas sobre espaços métricos Finsler. 
Definição 2.1. Dado um conjunto $X \neq \varnothing$, uma função $d: X \times X \longrightarrow \mathbb{R} e ́$ chamada uma métrica Finsler se as seguintes condições são cumpridas:

1. $d(p, q)=0$ se e somente se $p=q$,

2. existe $C>0$ tal que $d(p, q) \leqslant C d(q, p)$ para todo $p, q \in X$,

3. $d(p, q) \leqslant d(p, x)+d(x, q)$ para todo $p, x, q \in X$.

O par $(X, d)$ é chamado espaço métrico Finsler.

Espaços métricos usuais são exemplos imediatos de espaços métricos Finsler, mas no contexto desta tese valerá, em geral, a segunda condição acima, mais fraca que a simetria de uma métrica. Todo espaço métrico Finsler $(X, d)$ está automaticamente munido de uma métrica no sentido usual, dada por

$$
\bar{d}(p, q):=\frac{d(p, q)+d(q, p)}{2} .
$$

A função $d$ é contínua na topologia produto induzida por $\bar{d}$, em virtude da segunda condição da definição acima. Diremos que $(X, d)$ é completo se $(X, \bar{d})$ é completo como espaço métrico. O comprimento de uma curva $c:[a, b] \longrightarrow X$ é definido da maneira natural: seja $\Pi$ o conjunto de todos os subconjuntos finitos e ordenados de $[a, b]$. Tome $\pi=\left\{a=t_{0}, t_{1}, t_{2}, \ldots, t_{N}=b\right\} \in \Pi$ e

$$
s(\pi ; c):=\sum_{i=1}^{N} d\left(c\left(t_{i-1}\right), c\left(t_{i}\right)\right) .
$$

A curva $c$ será dita retificável quando

$$
L(c):=\sup _{\pi \in \Pi} s(\pi ; c)<\infty
$$

e o número $L(c)$ será o comprimento de $c$. A curva $c:[a, b] \longrightarrow X$ é uma geodésica quando $L\left(\left.c\right|_{[s, t]}\right)=t-s$ para todo $s, t \in[a, b]$ com $s<t$. Um espaço métrico Finsler completo $(X, d)$ é dito geodésico se dados dois pontos $p, q \in X$, existe uma geodésica $c:[a, b] \longrightarrow X \operatorname{com} c(a)=p$ e $c(b)=q$. Observe que em geral, $c$ não é única.

Definição 2.2. Sejam $A>0, B \geqslant 0,(X, d)$ e $\left(X^{\prime}, d^{\prime}\right)$ dois espaços métricos geodésicos Finsler. Uma função $f: X \longrightarrow X^{\prime}$ é uma $(A, B)$-quase isometria Finsler se

1. Existe $K>0$ tal que $d^{\prime}\left(x^{\prime}, f(X)\right) \leqslant K$ para todo $x^{\prime} \in X^{\prime}$.

2. para todo $p, q \in X$ vale

$$
\frac{1}{A} d(p, q)-B \leqslant \bar{d}(f(p), f(q)) \leqslant A d(p, q)+B .
$$


3. Existem $f^{\prime}: X^{\prime} \longrightarrow X$ e $Q>0$ tais que

$$
d\left(\left(f^{\prime} \circ f\right)(x), x\right) \leqslant Q
$$

para todo $x \in X e$

$$
\bar{d}\left(\left(f \circ f^{\prime}\right)\left(x^{\prime}\right), x^{\prime}\right) \leqslant Q
$$

para todo $x^{\prime} \in X^{\prime}$. A função $f^{\prime}$ é chamada quase-inversa de $f$.

Uma isometria é uma $(1,0)$-quase isometria $\operatorname{com} Q=0$. Qualquer quaseinversa de uma quase-isometria também é uma quase isometria.

Definição 2.3. Uma curva retificável $\gamma:[a, b] \longrightarrow X$ é uma $(A, B)$-quase geodésica se

$$
\frac{1}{A} d(\gamma(s), \gamma(t))-B \leqslant L\left(\gamma_{\mid[s, t]}\right) \leqslant A d(\gamma(s), \gamma(t))+B
$$

para todo $s, t \in[a, b]$ com $s<t$.

Das definições de quase-isometria e de $(A, B)$-quase geodésica, temos a seguinte consequência imediata.

Corolário 2.4. Sejam $(X, d)$ e $\left(X^{\prime}, d^{\prime}\right)$ espaços métricos geodésicos Finsler, $f: X \longrightarrow X^{\prime}$ uma $(A, B)$-quase-isometria e $\gamma:[a, b] \longrightarrow X$ uma geodésica. Então $\bar{\gamma}=f \circ \gamma$ é uma $\left(A^{\prime}, B^{\prime}\right)$-quase-geodésica de $\left(X^{\prime}, d^{\prime}\right)$.

Definição 2.5. Sejam $(M, d)$ um espaço métrico Finsler geodésico, completo, e $\gamma, \eta: \mathbb{R} \longrightarrow X$ geodésicas. Dizemos que $\beta$ e $\gamma$ são:

- f-assintóticas se existe $D=D(\gamma, \eta) \geqslant 0$ tal que $d(\gamma(t), \eta(t)) \leqslant D$ para todo $t \geqslant 0$

- b-assintóticas se existe $D=D(\gamma, \eta) \geqslant 0$ tal que $d(\gamma(t), \eta(t)) \leqslant D$ para todo $t \leqslant 0$.

- bi-assintóticas se $\gamma, \eta$ são simultaneamente f-assintóticas e b-assintóticas.

É claro que as relações de assintoticidade definidas acima são de equivalência. Para cada geodésica $\gamma: \mathbb{R} \longrightarrow X$, considere os conjuntos

$$
\gamma(+\infty):=\{\eta: \mathbb{R} \longrightarrow X / \gamma, \eta \text { são geodésicas f-assintóticas }\}
$$

e

$$
\gamma(-\infty):=\{\eta: \mathbb{R} \longrightarrow X / \gamma, \eta \text { são geodésicas b-assintóticas }\} .
$$

Denote por

$$
\partial X(+\infty):=\{\gamma(+\infty) / \gamma \text { é geodésica }\}
$$


$\mathrm{e}$

$$
\partial X(-\infty):=\{\gamma(-\infty) / \gamma \text { é geodésica }\}
$$

que chamaremos respectivamente de bordo positivo de Finsler-Gromov de $X$ e bordo negativo de Finsler-Gromov de $X$. Usaremos as notações

$$
X(+\infty):=X \cup \partial X(+\infty)
$$

e

$$
X(-\infty):=X \cup \partial X(-\infty)
$$

Vamos munir $X(+\infty)$ e $X(-\infty)$ de noções de convergência. Nossa referência será [11], Chapter 1, no qual o assunto é tratado em espaços métricos. Um raio geodésico generalizado positivo (respectivamente, negativo) é uma geodésica $\gamma:[0, R] \longrightarrow X$ onde $0<R \leqslant \infty$ (respectivamente, $\gamma:[R, 0] \longrightarrow X$ onde $-\infty \leqslant R<0)$. No caso em que $R<\infty$, consideraremos $\gamma(t)=\gamma(R)$ para $t \geqslant R$ (respectivamente, se $-\infty<R, \gamma(t)=\gamma(R)$ para $t \leqslant R$ ).

Note que os conjuntos de raios generalizados positivos e negativos são famílias equicontínuas. Para construir uma noção de convergência em $X(+\infty)$, fixe $p \in X$ e seja $x_{n} \in X(+\infty)$, usaremos a convergência uniforme em compactos.

Definição 2.6. Diremos que $x_{n} \rightarrow x \in X(+\infty)$ quando $n \rightarrow \infty$ se existe uma sequência $\gamma_{n}:\left[0, R_{n}\right] \longrightarrow X$ de raios generalizados positivos com $\gamma_{n}(0)=p, \gamma_{n}(+\infty)=x_{n}$ que converge uniformemente em compactos para um raio generalizado positivo $\gamma:[0, R] \longrightarrow X$ satisfazendo $\gamma(+\infty)=x$. Um subconjunto $B \subset X(+\infty)$ será fechado se

$$
x_{n} \in B \text { e } x_{n} \rightarrow x \quad \text { implica } \quad x \in B
$$

Analogamente, seja $\left\{x_{n}\right\} \subset X(-\infty)$.

Definição 2.7. Diremos que $x_{n} \rightarrow x \in X(-\infty)$ quando $n \rightarrow \infty$ se existe uma sequência $\gamma_{n}:\left[R_{n}, 0\right] \longrightarrow X$ de raios generalizados negativos com $\gamma_{n}(0)=p, \gamma_{n}(-\infty)=x_{n}$ que converge uniformemente em compactos para um raio generalizado negativo $\gamma:[R, 0] \longrightarrow X$ satisfazendo $\gamma(-\infty)=x$.Um subconjunto $B \subset X(-\infty)$ será fechado se

$$
x_{n} \in B \text { e } x_{n} \rightarrow x \quad \text { implica } \quad x \in B
$$

Estas noções de convergência serão usadas na seção seguinte e na subseção 3.1.1 para construir topologias que descrevam, de maneira razoável, os conjuntos $X(-\infty)$ e $X(+\infty)$. 


\section{2}

\section{Espaços Gromov-Finsler $\delta$-hiperbólicos}

Nesta seção, definiremos uma noção de $\delta$-hiperbolicidade para espaços métricos Finsler análoga à adotada por Gromov em [8], levando em conta a potencial falta de simetria da métrica Finsler em questão.

Sejam $(X, d)$ um espaço métrico Finsler geodésico e $x, y \in X$. Se $\gamma:[a, b] \longrightarrow X$ é uma geodésica tal que $\gamma(a)=x$ e $\gamma(b)=y$, o conjunto $\gamma([a, b]) \subset X$ será chamado de segmento geodésico ligando $x$ a $y$. Denote por $A_{x y}$ o conjunto de todos os segmentos geodésicos ligando $x$ a $y$ ou $y$ a $x$. Como a métrica não é necessariamente simétrica e pode haver mais de uma geodésica ligando $x$ a $y, A_{x y}$ em geral não é unitário. Observe que $A_{x y}=A_{y x}$.

Definição 2.8. Sejam $(X, d)$ um espaço métrico Finsler, $x_{0}, x_{1}, x_{2} \in X$. Fixados $\ell_{i, i+1} \in A_{x_{i} x_{i+1}}$, para $i=0,1,2$ (índices tomados módulo 3), o conjunto

$$
\Delta\left(x_{0}, x_{1}, x_{2}\right):=\ell_{01} \cup \ell_{12} \cup \ell_{20},
$$

é denominado triângulo geodésico de vértices $x_{0}, x_{1}, x_{2} e$ arestas $\ell_{i, i+1}$.

Definição 2.9. Um espaço métrico Finsler $(X, d)$ completo e geodésico é Gromov-Finsler $\delta$-hiperbólico se existe $\delta>0$ tal que para todo triângulo geodésico $\Delta\left(x_{0}, x_{1}, x_{2}\right)$, para todo $i \in\{0,1,2\}$ e para todo $x \in \ell_{i+2, i}$, valem:

1. $d\left(\ell_{i, i+1} \cup \ell_{i+1, i+2}, x\right) \leqslant \delta e$

2. $d\left(x, \ell_{i, i+1} \cup \ell_{i+1, i+2}\right) \leqslant \delta$,

com incides tomados módulo 3.

Geometricamente, cada aresta de um triângulo geodésico qualquer de vértices $x_{0}, x_{1}, x_{2}$ está dentro da "vizinhança tubular" de "raio $\delta$ " da união das demais duas, como mostra a Figura 2.1 (a noção de "raio" não é precisa, pois a distância não é simétrica). 


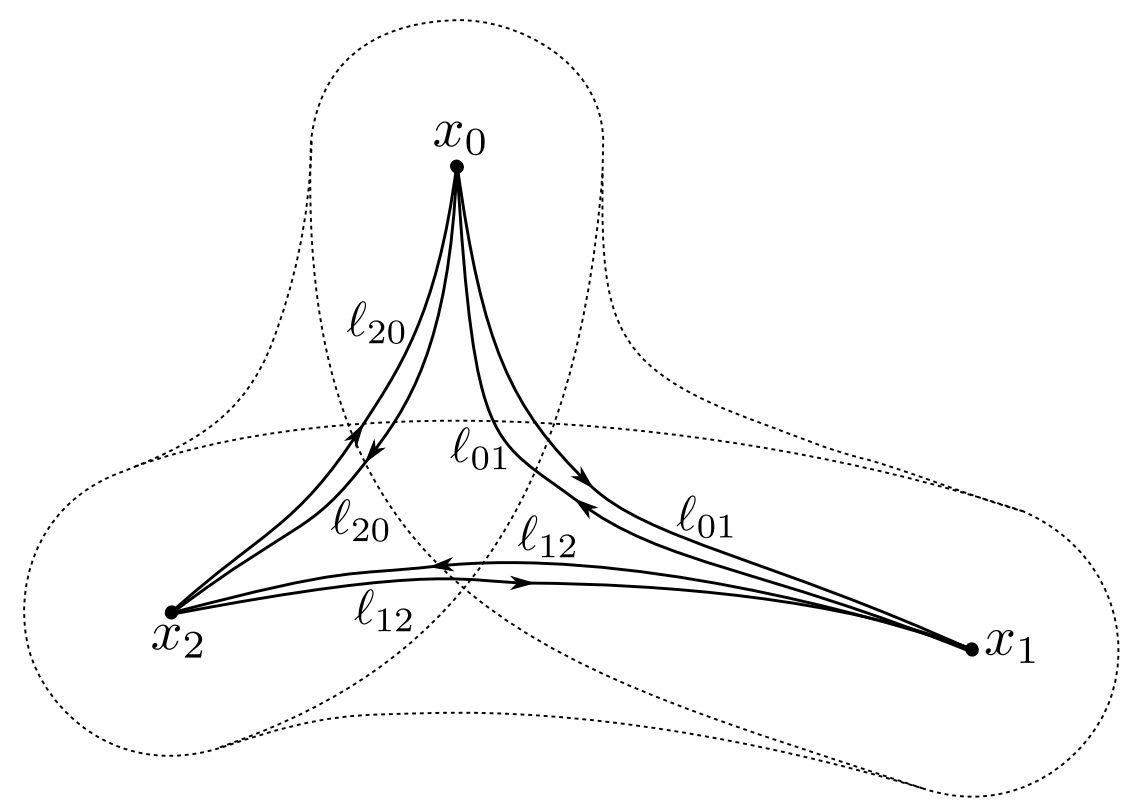

Figura 2.1: Independentemente da escolha de $\ell_{i j}, \ell_{j k}, \ell_{k i}$, a aresta $\ell_{k i}$ está contida na "vizinhança $\delta$-tubular" de $\ell_{i j} \cup \ell_{j k}$

Observação 2.10. Dada uma geodésica $\gamma:[a, b] \longrightarrow X$ ligando $x$ a $y$, usaremos o símbolo $[x, y]$ para denotar o conjunto $\gamma([a, b])$, independentemente da escolha de $\gamma$. Este abuso de notação não trará prejuízos no decorrer do trabalho. Em particular, se $(M, F)$ não tem pontos conjugados, então dados $x, y \in \tilde{M}$ a notação $[x, y]$ não é ambígua, pois as imagens de todas as geodésica ligando $x$ a $y$ coincidem.

Quando $(X, d)$ é Gromov-Finsler $\delta$-hiperbólico, as noção de convergência definida anteriormente permite munir $X(+\infty)$ e $X(-\infty)$ de topologias como descrevemos abaixo.

Proposição 2.11. Seja $(X, d)$ um espaço métrico Finsler geodésico, próprio e Gromov-Finsler $\delta$-hiperbólico. Então,

1. Os complementos dos fechados descritos nas Definições 2.6 e 2.7 acima formam bases para topologias em $X(+\infty)$ e $X(-\infty)$ respectivamente.

2. As topologias assim geradas independem da escolha do ponto $p$.

3. As inclusões $X \subset X(+\infty)$ e $X \subset X(-\infty)$ são homeomorfismos sobre $X$.

4. $\partial X(+\infty) \subset X(+\infty)$ e $\partial X(-\infty) \subset X(-\infty)$ são compactos.

Demonstração. Ver [14], Proposition 3.7. 
As quase-isometrias preservam a $\delta$-hiperbolicidade, como enunciamos abaixo.

Proposição 2.12. Sejam $(X, d)$ e $\left(X^{\prime}, d^{\prime}\right)$ espaços métricos geodésicos Finsler e $f: X \longrightarrow X^{\prime}$ uma $(A, B)$-quase-isometria. Então $(X, d)$ é Gromov-Finsler $\delta$ hiperbólico se e somente se $\left(X^{\prime}, d^{\prime}\right)$ é Gromov-Finsler $\delta^{\prime}$-hiperbólico para algum $\operatorname{par} \delta, \delta^{\prime} \in \mathbb{R}^{+}$.

Demonstração. A prova é análoga ao caso dos espaços métricos usuais $\delta$ hiperbólicos e pode ser encontrada, por exemplo, em [14], p.402.

Dada uma $(A, B)$-quase-geodésica $\gamma:[0, \infty) \longrightarrow X$ de um espaço Gromov-Finsler $\delta$-hiperbólico e próprio, podemos dar sentido ao símbolo $\gamma(+\infty)$ da seguinte forma: fixe $p \in X$, tome geodésicas ligando $p$ a $\gamma(n)$ para $n \in \mathbb{N}$ e, usando o teorema de Arzelá-Ascoli, construa um raio geodésico $\beta:[0, \infty) \longrightarrow X$. Defina $\gamma(+\infty):=\beta(+\infty)$. Da definição de quase-geodésica e da $\delta$-hiperbolicidade de $X, \gamma(+\infty)$ independe de $p$ e do limite $\beta$ construido.

Teorema 2.13. Sejam $(X, d)$ e $\left(X^{\prime}, d^{\prime}\right)$ espaços métricos Finsler geodésicos, próprios e Gromov-Finsler $\delta$-hiperbólicos. Se $f: X \longrightarrow X^{\prime}$ é uma $(A, B)$ quase-isometria, então a aplicação $f: \partial X \longrightarrow \partial X^{\prime}$ dada por

$$
\gamma(+\infty) \longmapsto(f \circ \gamma)(+\infty)
$$

é um homeomorfismo com respeito à topologia definida acima.

Demonstração. Novamente referimos ao leitor [14], Theorem 3.9.

Antes de prosseguirmos com a exposição, observamos que, nas topologias descritas pelas Definições 2.6 e 2.7, uma isometria de $X$ pode ser estendida a um homeomorfismo de $X(+\infty)$ e um de $X(-\infty)$.

Proposição 2.14. Sejam $(X, d)$ um espaço métrico Gromov-Finsler $\delta$ hiperbólico e próprio. Se $\phi: X \longrightarrow X$ é uma isometria, então as aplicações $\bar{\phi}: X(+\infty) \longrightarrow X(+\infty)$ e $\bar{\phi}: X(-\infty) \longrightarrow X(-\infty)$ dadas respectivamente por

$$
\bar{\phi}(\gamma(+\infty)):=(\phi \circ \gamma)(+\infty)
$$

$e$

$$
\bar{\phi}(\gamma(-\infty)):=(\phi \circ \gamma)(-\infty)
$$

são homeomorfismos. 


\section{3}

\section{Visiblidade em variedades Finsler}

Em uma variedade Riemanniana, a noção de ângulo entre vetores tangentes é canônica, pois em cada espaço tangente há um produto interno. Isso permite fornecer uma definição natural de visibilidade como em [7]. Em variedades Finsler, não há um conceito de ângulo canonicamente associado à métrica Finsler e, portanto, o mesmo se passa com a visibilidade. Sugerimos nesta seção uma definição de visibilidade usando o ângulo de Landsberg, que definiremos a seguir. Com ela, conseguiremos estender para variedades Finsler a conhecida [24] equivalência entre $\delta$-hiperbolicidade e visibilidade em variedades Riemannianas.

Sejam $(M, F)$ uma variedade Finsler e $(\tilde{M}, \tilde{F})$ seu recobrimento universal, com a estrutura Finsler pull-back de $F$ por $\pi: \tilde{M} \longrightarrow M$. Lembremos que cada espaço tangente está munido de uma métrica Riemanniana $\left(g_{i j}\right)$ e, consequentemente, $\left(\mathcal{S}_{p},\left(g_{i j}\right)\right)$ é uma variedade Riemanniana para cada $p \in M$, com função distância que denotaremos por $d_{\mathcal{S}_{p}}$.

Definição 2.15. Sejam $(M, F)$ uma variedade Finsler e $v, w \in T_{p} M$ vetores não-nulos. $O$ ângulo de Landsberg entre $v$ e w é o número real

$$
\varangle_{p}(v, w)=d_{\mathcal{S}_{p}}\left(\frac{v}{F(p, v)}, \frac{w}{F(p, w)}\right) .
$$

Como $d_{\mathcal{S}_{p}}$ é uma distância Riemanniana, temos que $\varangle_{p}(v, w)=\varangle_{p}(w, v)$. Além disso, $\varangle_{p}(v, w)=0$ se e somente se $v=\lambda w$ para algum $\lambda>0$. Cabe observar que $\varangle_{p}(v,-v)$ não necessariamente é $\pi$, mas se $M$ é compacta, existe $\kappa>0$ tal que $\varangle_{p}(v,-v) \geqslant \kappa$ para todo $(p, v) \in \mathcal{S} M$.

Provaremos a seguir que o ângulo de Landsberg é um invariante por isometrias Finsler. Este resultado é importante para que possamos adaptar os argumentos de Eberlein (particularmente a demonstração da proposição 2.6 de [6]) e provar a transitividade do fluxo geodésico no contexto Finsler. Embora a demonstração da invariância do ângulo de Landsberg seja elementar, decidimos acrescentá-la aqui por nos parecer pouco comum sua apresentação mesmo em textos introdutórios à geometria Finsler.

Lema 2.16. Seja $(M, F)$ uma variedade Finsler. Para toda isometria $\phi$ : $M \longrightarrow M$ e para todo $v, w \in T_{p} M$, vale

$$
\varangle_{p}(v, w)=\varangle_{\phi(p)}\left(d \phi_{p}(v), d \phi_{p}(w)\right) .
$$

Demonstração. Por hipótese, $F(p, w)=F\left(\phi(p), d \phi_{p}(w)\right)$ para todo $w \in T_{p} M$ e portanto $\left(\phi, d \phi_{p}\right)$ leva $\mathcal{S}_{p}$ difeomorficamente em $\mathcal{S}_{\phi(p)}$. Se fixarmos um sistema 
de coordenadas locais em torno de $p$, digamos $\left(x_{1}, \ldots, x_{n}\right)$, um vetor $w \in T_{p} M$ será da forma

$$
w^{1} \frac{\partial}{\partial x_{1}}+\cdots+w^{n} \frac{\partial}{\partial x_{n}} .
$$

Como $d \phi_{p}: T_{p} M \longrightarrow T_{\phi(p)} M$ é um isomorfismo, denotando

$$
\frac{\partial}{\partial \bar{x}_{1}}:=d \phi_{p}\left(\frac{\partial}{\partial x_{1}}\right)
$$

temos que

$$
d \phi_{p}(w)=w^{1} \frac{\partial}{\partial \bar{x}_{1}}+\cdots+w^{n} \frac{\partial}{\partial \bar{x}_{n}} .
$$

Ou seja, se $\mathbf{x}: U \subset M \longrightarrow \mathbb{R}^{n}$ é um sistema local de coordenadas em torno de $p \in M$, então no sistema de coordenadas $\overline{\mathbf{x}}=\mathbf{x} \circ \phi$ em torno de $\phi(p)$, as coordenadas tangentes são invariantes por $(\phi, d \phi)$. Em particular

$$
\left(\frac{1}{2} \frac{\partial^{2}}{\partial y^{i} \partial y^{j}} F^{2}\right)_{(p, w)}=\left(\frac{1}{2} \frac{\partial^{2}}{\partial y^{i} \partial y^{j}} F^{2}\right)_{\left(\phi(p), d \phi_{p}(w)\right.}
$$

e portanto

$$
\left(g_{i j}\right)_{(p, w)}=\left(g_{i j}\right)_{\left(\phi(p), d \phi_{p}(w)\right)} .
$$

Isso implica que $d \phi_{p}$ é uma isometria Riemanniana entre $\mathcal{S}_{p}$ e $\mathcal{S}_{\phi(p)}$. Então, dados $w_{1}, w_{2} \in \mathcal{S}_{p}$, temos

$$
d_{\mathcal{S}_{p}}\left(w_{1}, w_{2}\right)=d_{\mathcal{S}_{\phi(p)}}\left(d \phi_{p}\left(w_{1}\right), d \phi_{p}\left(w_{2}\right)\right)
$$

como queríamos demonstrar.

A seguinte definição é uma versão Finsler da noção de visibilidade, análoga àquela adotada por Eberlein por exemplo em [6] e em [7], e leva em conta a potencial falta de simetria de $d_{F}$.

Definição 2.17. Seja $(M, F)$ uma variedade Finsler completa sem pontos conjudados. Dizemos que $(M, F)$ é uma variedade de visibilidade se para todo $\varepsilon>0$ e para todo $p \in \tilde{M}$ existe $R=R(\varepsilon, p)>0$ satisfazendo aos seguintes axiomas:

1. Se $\beta, \gamma$ são duas geodésicas unitárias com $\beta(0)=\gamma(0)=p$ e existem $0 \leqslant s, t$ satisfazendo

$$
d_{F}(p,[\beta(s), \gamma(r)]) \geqslant R \quad \text { e } \quad d_{F}(p,[\gamma(r), \beta(s)]) \geqslant R,
$$

então $\varangle_{p}\left(\beta^{\prime}(0), \gamma^{\prime}(0)\right) \leqslant \varepsilon$. 
2. Se $\eta, \sigma$ são duas geodésicas unitárias com $\eta(0)=\sigma(0)=p$ e existem $a, b \leqslant 0$ satisfazendo

$$
d_{F}(p,[\eta(a), \sigma(b)]) \geqslant R \quad \text { e } \quad d_{F}(p,[\sigma(b), \eta(a)]) \geqslant R
$$

então $\varangle_{p}\left(\eta^{\prime}(0), \sigma^{\prime}(0)\right) \leqslant \varepsilon$.

$(M, F)$ é de visibilidade uniforme se $R=R(\varepsilon)$.

\section{4}

\section{Bígonos geodésicos são estreitos}

Dados $p, q \in \tilde{M}$, sabemos que em geral $[p, q] \neq[q, p]$. Mostraremos nesta seção que é possível controlar uniformemente em $\tilde{M}$ o quão "longe" estão os segmentos $[p, q]$ e $[q, p]$ um do outro. Este controle será obtido como consequência de propriedades geométricas de $(\tilde{M}, \tilde{F})$ e será uma das peças fundamentais para que possamos mostrar a equivalência entre $\delta$-hiperbolicidade e visibilidade Finsler na próxima seção.

Definição 2.18 (bígonos - Figura 2.2). Sejam $(M, F)$ uma variedade Finsler sem pontos conjugados e de visibilidade. Dados $p, q \in \tilde{M}$, o bígono ligando $p, q$ é o conjunto $[p, q] \cup[q, p]$.

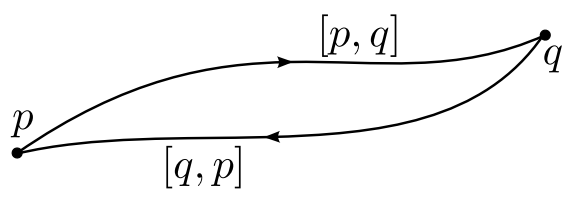

Figura 2.2: O bígono ligando $p$ e $q$

Fixemos algumas notações: sejam $(M, F)$ uma variedade Finsler sem pontos conjugados e $(\tilde{M}, \tilde{F})$ seu recobrimento universal. Dados $x \in \tilde{M}$ e $r>0$, consideraremos as pseudo-bolas

$$
B_{r}^{-}(x):=\left\{p \in \tilde{M} / d_{\tilde{F}}(p, x)<r\right\} \quad \text { e } \quad B_{r}^{+}(x):=\left\{p \in \tilde{M} / d_{\tilde{F}}(x, p)<r\right\},
$$

além das respectivas pseudo-esferas geodésicas definidas por

$$
S_{r}^{-}(x):=\left\{p \in \tilde{M} / d_{\tilde{F}}(p, x)=r\right\} \quad \text { e } \quad S_{r}^{+}(x):=\left\{p \in \tilde{M} / d_{\tilde{F}}(x, p)=r\right\}
$$

e também as pseudo-bolas fechadas

$$
\overline{B_{r}^{-}(x)}:=B_{r}^{-}(x) \cup S_{r}^{-}(x) \quad \text { e } \quad \overline{B_{r}^{+}(x)}:=B_{r}^{+}(x) \cup S_{r}^{+}(x) .
$$


Fixe $y \in \tilde{M}$. Para cada $x \in \tilde{M}$, seja $\nu_{y}(x)$ o vetor unitário tangente em $x$ à geodésica que liga $x$ a $y$. Pelo lema de Gauss para variedades Finsler (ver por exemplo [20]), $\nu_{y}(x)$ é ortogonal, com respeito à métrica adaptada de $T \tilde{M}-\{0\}$, à pseudo-esfera $S_{r}^{-}(y)=\left\{p \in \tilde{M} / d_{\tilde{F}}(p, y)=r\right\}$, onde $r=d_{\tilde{F}}(x, y)$. Como a norma do operador de curvatura das pseudo-esferas $S_{r}^{-}(y)$ é limitada superiormente para $r \geqslant 1$, já que a curvatura normal é solução da equação de Ricatti e $M$ não tem pontos conjugados (para uma versão Riemanniana deste fato, da qual a versão Finsler segue diretamente usando a conexão de Chern, ver por exemplo [25]), então cada campo $\nu_{y}$ é Lipschitz em $\tilde{M}-\overline{B_{1}^{-}(y)}$ e a constante de Lipschitz, que denotaremos por $L$, independe de $y$ (para um tratamento extenso sobre estimativas de operadores de curvatura em variedades Finsler, consulte por exemplo [26] - particularmente p.221 a p.224).

Para mostrar que a "largura" dos bígonos de $(\tilde{M}, \tilde{F})$ está controlada, precisaremos do seguinte lema técnico.

Lema 2.19. Seja $(M, F)$ uma variedade Finsler compacta, sem pontos conjugados e de visibilidade uniforme. Dado $\varepsilon>0$, existem $N=N(\varepsilon)$ e $\delta=\delta(L)$ com a seguinte propriedade: se $\gamma$ é uma geodésica unitária de $\tilde{M}, x \in \tilde{M}$ satisfaz $d_{\tilde{F}}(x, \gamma)>N$ e $p \in B_{\delta}^{+}(x)$, então $\varangle_{p}\left(\nu_{y}(p), \nu_{z}(p)\right) \leqslant \varepsilon$ para todo $y, z \in \gamma$.

Demonstração. Sejam $y, z \in \gamma$. Como $\tilde{M}$ é de visibilidade, seja $N>1$ tal que se $d_{\tilde{F}}(x, \gamma)>N$, tenhamos $\varangle_{x}\left(\nu_{z}(x), \nu_{y}(x)\right)=d_{\mathcal{S}}\left(\nu_{z}(x), \nu_{y}(x)\right) \leqslant \frac{\varepsilon}{2}$. Como os campos $\nu_{z}$ são $L$-Lipschitz, dado $p \in \tilde{M}$,

$$
\begin{aligned}
\varangle_{p}\left(\nu_{z}(p), \nu_{y}(p)\right) & =d_{\mathcal{S}}\left(\nu_{z}(p), \nu_{y}(p)\right) \\
& \leqslant d_{\mathcal{S}}\left(\nu_{z}(p), \nu_{z}(x)\right)+d_{\mathcal{S}}\left(\nu_{z}(x), \nu_{y}(x)\right)+d_{\mathcal{S}}\left(\nu_{y}(x), \nu_{y}(p)\right) \\
& \leqslant L d_{\tilde{F}}(p, x)+\frac{\varepsilon}{2}+L d_{\tilde{F}}(x, p) \\
& \leqslant 2 L C d_{\tilde{F}}(x, p)+\frac{\varepsilon}{2},
\end{aligned}
$$

onde $C$ é a constante definida em (1-3). Tomando então $\delta=\frac{\varepsilon}{4 L C}$, segue que se $p \in B_{\delta}^{+}(x)$ e $d_{\tilde{F}}(x, \gamma)>N$, então $\varangle_{p}\left(\nu_{z}(p), \nu_{y}(p)\right) \leqslant \varepsilon$, como queríamos demonstrar.

Proposição 2.20 (bígonos são estreitos). Seja $(M, F)$ uma variedade Finsler compacta, sem pontos conjugados e de visibilidade uniforme. Então, existe $A>0$ tal que para todo $p, q \in \tilde{M}$ valem:

1. $d_{\tilde{F}}(x,[p, q]) \leqslant$ A para todo $x \in[q, p]$.

2. $d_{\tilde{F}}(x,[q, p]) \leqslant$ A para todo $x \in[p, q]$. 
Demonstração. Suponha que $(\tilde{M}, \tilde{F})$ não satisfaz o enunciado e considere uma sequência $\left[a_{n}, b_{n}\right] \subset \tilde{M}$ de geodésicas tais que (Figura 2.3):

- $\gamma_{n}: \mathbb{R} \longrightarrow \tilde{M}$ é a geodésica unitária que contém $\left[a_{n}, b_{n}\right]$;

- $\sigma_{n}: \mathbb{R} \longrightarrow \tilde{M}$ é a geodésica unitária que contém $\left[b_{n}, a_{n}\right]$;

$-\rho_{n}:=\max \left\{d_{\tilde{F}}\left(z,\left[a_{n}, b_{n}\right]\right), z \in\left[b_{n}, a_{n}\right]\right\} \geqslant n ;$

- $x_{n} \in\left[b_{n}, a_{n}\right]$ e $y_{n} \in\left[a_{n}, b_{n}\right]$ são tais que $\rho_{n}=d_{\tilde{F}}\left(x_{n}, y_{n}\right)$;

$-\gamma_{n}(0)=y_{n}$ e $\sigma_{n}(0)=x_{n}$

- $\eta_{n}: \mathbb{R} \longrightarrow \tilde{M}$ é a geodésica unitária que contém $\left[x_{n}, y_{n}\right]$ com $\eta_{n}(0)=x_{n}$;

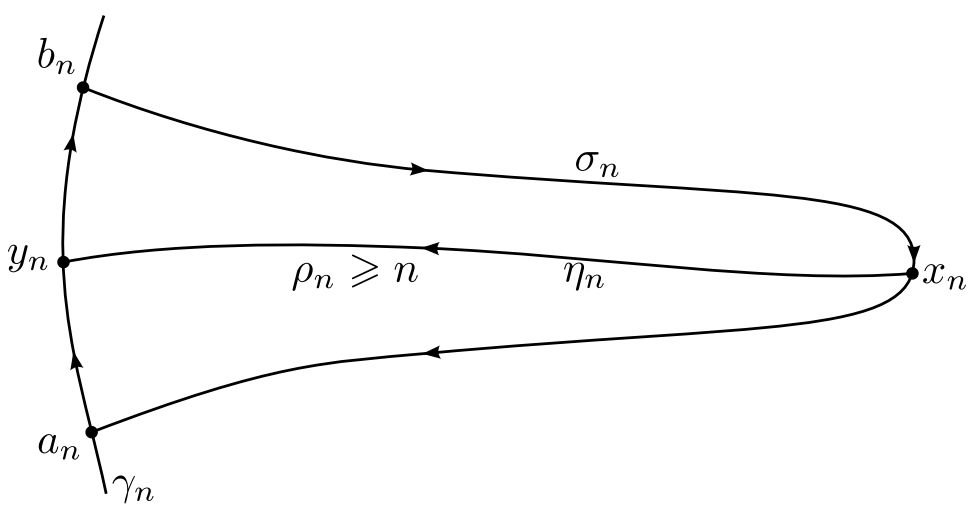

Figura 2.3: Bígonos arbitrariamente "largos"

Usando transformações de recobrimento, podemos supor (a menos de considerarmos uma subsequência) que $x_{n} \rightarrow x \in \tilde{M}$. Dado $\varepsilon>0$, sejam $N>1$ e $\delta>0$ dados pelo lema anterior. Considere $B_{\delta}^{+}(x)$ e $n_{1}$ tal que $x_{n} \in B_{\delta}^{+}(x)$ para $n \geqslant n_{1}$. Como $\rho_{n} \rightarrow \infty$, existe $n_{2}$ tal que $n \geqslant n_{2}$ implica que $d_{\tilde{F}}\left(x_{n},\left[a_{n}, b_{n}\right]\right) \geqslant N$. Daqui até o fim da demonstração, $n \geqslant \max \left\{n_{1}, n_{2}\right\}$.

As pseudo-esferas geodésicas $S_{\rho_{n}}^{-}\left(y_{n}\right)$ têm curvatura normal limitada e, portanto (diminuindo $\delta$ se necessário), existe uma família de cartas locais $\varphi_{n}: B_{\delta}^{+}\left(x_{n}\right) \longrightarrow \mathbb{R}^{n}=\mathbb{R}^{n-1} \times \mathbb{R}^{1}$ tal que:

1. $\varphi_{n}\left(x_{n}\right)=(0, \ldots, 0)$,

2. $\left(d \varphi_{n}\right)_{x_{n}}\left(-\nu_{y_{n}}\left(x_{n}\right)\right)=(0, \ldots, 0,1)$,

3. as segundas derivadas de $\varphi_{n}$ são uniformemente limitadas em $n$ na pseudo-bola aberta $B_{\delta}^{+}\left(x_{n}\right)$.

Afirmamos que:

af1) $\sigma_{n}(t) \in B_{\delta}^{+}\left(x_{n}\right)$ para todo $t \in(-\delta / C, \delta)$.

Lembramos que $\sigma_{n}$ é uma geodésica minimizante ligando $b_{n}$ a $a_{n}$. Se $t \in(-\delta / C, 0]$, então $d_{\tilde{F}}\left(x_{n}, \sigma_{n}(t)\right) \leqslant C d_{\tilde{F}}\left(\sigma_{n}(t), x_{n}\right)=C|t|<\delta$. Se $t \in[0, \delta)$, então $d_{\tilde{F}}\left(x_{n}, \sigma_{n}(t)\right)=t<\delta$. 
af2) Para cada $s \in \mathbb{R}$, seja $\rho_{n}(s):=d_{\tilde{F}}\left(x_{n}, \gamma_{n}(s)\right)$. Então $x_{n} \in S_{\rho_{n}(s)}^{-}(\gamma(s))$.

Isso segue diretamente da definição de $\rho_{n}(s)$.

af3) Para cada $p \in\left[b_{n}, a_{n}\right]$, existe $q \in\left[a_{n}, b_{n}\right]$ tal que $d_{\tilde{F}}(p, q) \leqslant d_{\tilde{F}}\left(x_{n}, q\right)$.

Basta notar que, fixado $p \in\left[b_{n}, a_{n}\right]$,

$$
\begin{aligned}
d_{\tilde{F}}\left(x_{n}, y_{n}\right) & \left.=\max \left\{d_{\tilde{F}}\left(z,\left[a_{n}, b_{n}\right]\right) / z \in\left[b_{n}, a_{n}\right]\right)\right\} \\
& \geqslant d_{\tilde{F}}\left(p,\left[a_{n}, b_{n}\right]\right) \\
& =d_{\tilde{F}}(p, q)
\end{aligned}
$$

para algum $q \in\left[a_{n}, b_{n}\right]$. Ao mesmo tempo,

$$
d_{\tilde{F}}\left(x_{n}, y_{n}\right)=d_{\tilde{F}}\left(x_{n},\left[a_{n}, b_{n}\right]\right) \leqslant d_{\tilde{F}}\left(x_{n}, q\right) .
$$

af4) $\sigma_{n}((-\delta / c, \delta)) \subset \cup_{s \in \mathbb{R}} \overline{B_{\rho_{n}(s)}^{-}(\gamma(s))}$.

Como $\sigma_{n}(t) \in\left[b_{n}, a_{n}\right]$ para $t \in(-\delta / C, \delta)$, a af3 diz que existe $q=\gamma_{n}(s)$ tal que $d_{\tilde{F}}\left(\sigma_{n}(t), \gamma_{n}(s)\right) \leqslant d_{\tilde{F}}\left(x_{n}, \gamma_{n}(s)\right)=\rho_{n}(s)$, definido da af2 (Figura $2.4)$.

af5) $\sigma_{n}(-\delta / C, \delta) \subset \cup_{s \in \mathbb{R}}\left(\overline{B_{\rho_{n}(s)}^{-}\left(\gamma_{n}(s)\right)} \cap B_{\delta}^{+}\left(x_{n}\right)\right)$.

Segue de af1 e de aff.

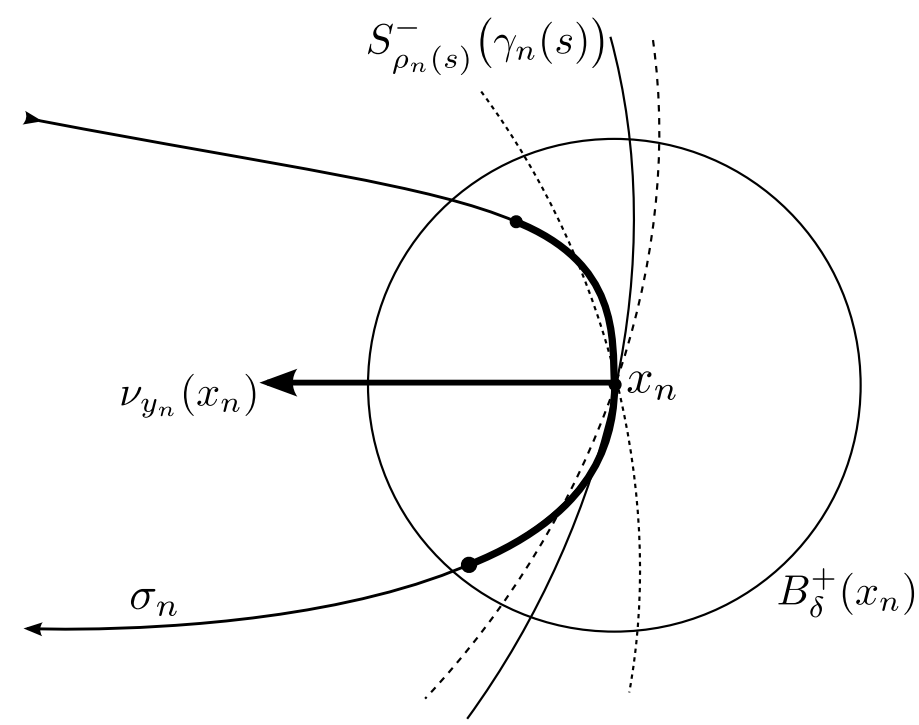

Figura 2.4: A união das pseudo-bolas $\overline{B_{\rho_{n}(s)}^{-}(\gamma(s))}$ contém $\sigma_{n}((-\delta / C, \delta))$.

Para cada $n \geqslant n_{0}$, as subvariedades mergulhadas $S_{\rho_{n}(s)}^{-}(\gamma(s)) \cap B_{\delta}^{+}\left(x_{n}\right)$ são representadas pelas cartas $\varphi_{n}$ por gráficos em $\mathbb{R}^{n-1} \times \mathbb{R}^{1}$ passando por $(0, \ldots, 0)$, com curvatura limitada uniformemente em $s \in \mathbb{R}$, pois $\rho_{n}(s) \geqslant$ 
$\rho_{n} \geqslant N$. Pelo Lema $2.19, \varangle_{p}\left(\nu_{y_{n}}(p), \nu_{\gamma_{n}(s)}(p)\right) \leqslant \varepsilon$ para todo $p \in B_{\delta}^{+}(x)$. Em particular,

$$
\varangle_{p}\left(\nu_{y_{n}}\left(x_{n}\right), \nu_{\gamma_{n}(s)}\left(x_{n}\right)\right) \leqslant \varepsilon .
$$

Portanto, as imagens de $S_{\rho_{n}(s)}^{-}\left(\gamma_{n}(s)\right) \cap B_{\delta}^{+}\left(x_{n}\right)$ pelas cartas $\varphi_{n}$ são gráficos de funções $L$-Lipschitz (com $L$ independente de $n$ e de $s$ ) em $\mathbb{R}^{n-1} \times \mathbb{R}^{1}$ passando por $(0, \ldots, 0)$, para todo $s \in(-\delta / C, \delta)$. Em particular,

$$
\varphi_{n}\left(\overline{B_{\rho_{n}(s)}^{-}(\gamma(s))} \cap B_{\delta}^{+}\left(x_{n}\right)\right)
$$

está abaixo do cone positivo centrado no eixo $(0, \ldots, 0,1)$, com vértice na origem e fazendo ângulo $o(\operatorname{arctg}(L))$ com o hiperplano $\left(x_{1}, \ldots, x_{n-1}, 0\right)$ (Figura 2.5).

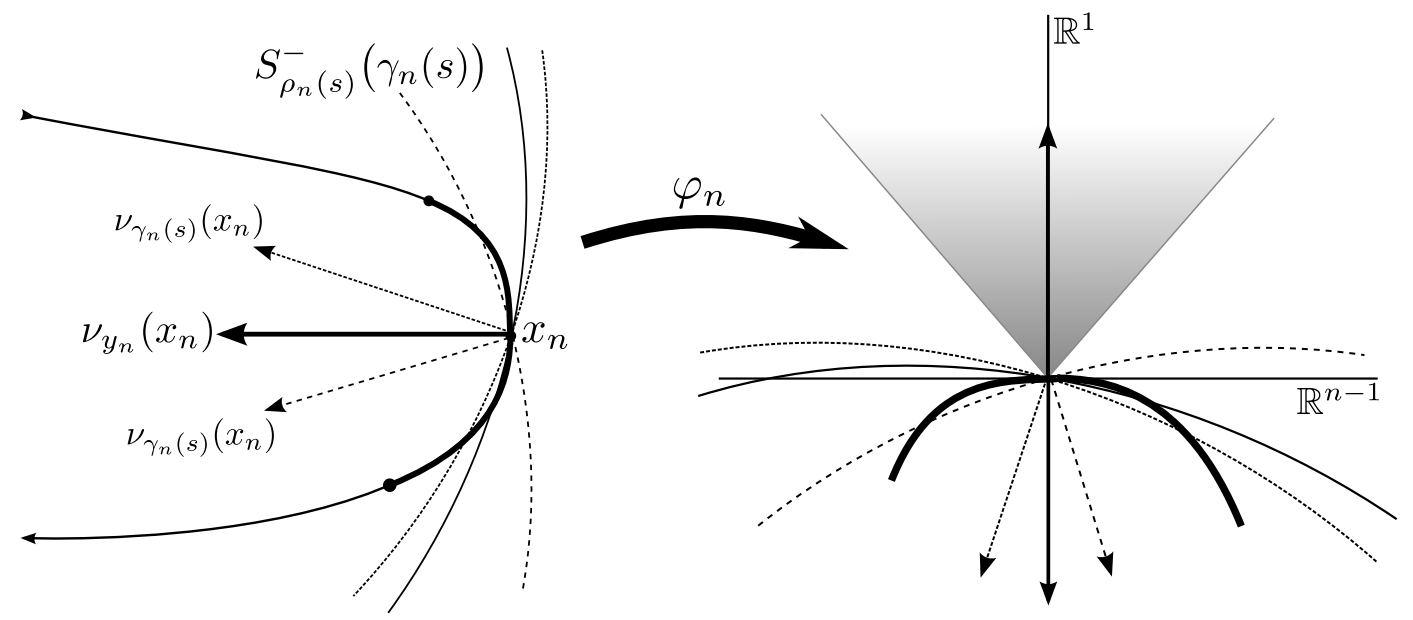

Figura 2.5: Imagem de $\overline{B_{\rho_{n}(s)}^{-}(\gamma(s))} \cap B_{\delta}^{+}\left(x_{n}\right)$ abaixo de um cone

Por outro lado, pela visibilidade Finsler, para $n$ suficientemente grande, $\varangle_{x_{n}}\left(\nu_{y_{n}}\left(x_{n}\right), \sigma_{n}^{\prime}(0)\right)$ é pequeno e portanto, para $t$ próximo de 0 , a imagem de $\sigma_{n}$ por $\varphi_{n}$ está próxima do eixo vertical $(0, \ldots, 0,1)$. Mas isso implica que algum trecho de $\varphi_{n}\left(\sigma_{n}(-\delta / C, \delta)\right)$ não está abaixo do cone mencionado anteriormente, já que $\varphi_{n}\left(\sigma_{n}(-\delta / C, \delta)\right)$ está próxima do eixo vertical para $t$ próximo de zero no intervalo $(-\delta / C, \delta)$. Essa contradição mostra que não pode ocorrer

$$
\rho_{n}=\max \left\{d_{\tilde{F}}\left(z,\left[a_{n}, b_{n}\right]\right), z \in\left[b_{n}, a_{n}\right]\right\} \geqslant n .
$$

Concluímos então que existe $A \geqslant 0$ tal que $d_{\tilde{F}}(x,[p, q]) \leqslant A$ para todo $x \in[q, p]$. Usando que $d_{\tilde{F}}(x, y) \leqslant C d_{\tilde{F}}(y, x)$, encerramos a demonstração da proposição. 


\section{5}

\section{Visibilidade é equivalente à $\delta$-hiperbolicidade}

Daqui em diante, precisaremos do seguinte conceito de divergência de geodésicas que se encontram em um ponto.

Definição 2.21. Sejam $\beta, \gamma: \mathbb{R} \longrightarrow X$ geodésicas com $\beta(0)=\gamma(0)=p$. Dizemos que $\beta$ e $\gamma$ divergem uniformemente para $t>0$ (respectivamente, $t<0)$ se para todo $\varepsilon>0$ e para todo $R>0$ existe $t_{0}=t_{0}(R)>0 \mathrm{com}$ a seguinte propriedade: se $\varangle_{p}\left(\gamma^{\prime}(0), \beta^{\prime}(0)\right) \geqslant \varepsilon$, então $d(\beta(t), \gamma(t)) \geqslant R$ para todo $t>t_{0}$ (respectivamente, para todo $t<t_{0}$ ).

Enunciamos a seguir o resultado que estabelece a referida relação entre a visibilidade e a $\delta$-hiperbolicidade. Ele é motivado pelo análogo Riemanniano, do qual o enunciado abaixo e sua prova são adaptações naturais (ver por exemplo [24], Theorem 6.8).

Teorema 2.22. Seja $(M, F)$ uma variedade Finsler de classe $C^{\infty}$ compacta e sem pontos conjugados. Então $(\tilde{M}, \tilde{F})$ é de visibilidade uniforme se e somente se $(\tilde{M}, \tilde{F})$ é um espaço Gromov-Finsler $\delta$-hiperbólico para algum $\delta>0$ e os raios geodésicos divergem no futuro e no passado.

Subdividiremos o enunciado deste teorema (e também sua demonstração) em dois lemas. Observamos primeiramente que as divergências para $t<0 \mathrm{e}$ $t>0$ dos raios geodésicos seguem diretamente da noção de visibilidade Finsler. Usaremos fortemente a Proposição 2.20 provada acima.

\subsection{1}

\section{Visibilidade implica $\delta$-hiperbolicidade}

Lema 2.23. Seja $(M, F)$ uma variedade Finsler de classe $C^{\infty}$ sem pontos conjugados. Se $(\tilde{M}, \tilde{F})$ é de visibilidade uniforme, então existe $\delta>0$ tal que $\left(\tilde{M}, d_{\tilde{F}}\right)$ é um espaço Gromov-Finsler $\delta$-hiperbólico.

Demonstração. Queremos provar que todo triângulo geodésico satisfaz aos axiomas da Definição 2.9. Pela proposição 2.20 , podemos mostrar que esses axiomas valem para triângulos da forma $\Delta=\left[x_{0}, x_{1}\right] \cup\left[x_{1}, x_{2}\right] \cup\left[x_{2}, x_{0}\right]$, pois os segmentos $\left[x_{1}, x_{0}\right],\left[x_{2}, x_{1}\right]$ e $\left[x_{0}, x_{2}\right]$ estão a uma distância controlada dos seus respectivos lados de $\Delta$. Suponhamos por contradição que existe uma sequência de triângulos geodésicos

$$
\Delta_{n}=\left[x_{0}^{n}, x_{1}^{n}\right] \cup\left[x_{1}^{n}, x_{2}^{n}\right] \cup\left[x_{2}^{n}, x_{0}^{n}\right]
$$


e $p_{n} \in\left[x_{0}^{n}, x_{1}^{n}\right]$ tais que

$$
d_{\tilde{F}}\left(p_{n},\left[x_{1}^{n}, x_{2}^{n}\right] \cup\left[x_{2}^{n}, x_{0}^{n}\right]\right) \geqslant n
$$

para todo $n \in \mathbb{N}$. Considere os triângulos geodésicos

$$
\Delta_{n}^{1}=\left[x_{0}^{n}, p_{n}\right] \cup\left[p_{n}, x_{2}^{n}\right] \cup\left[x_{2}^{n}, x_{0}^{n}\right]
$$

e

$$
\Delta_{n}^{2}=\left[p_{n}, x_{1}^{n}\right] \cup\left[x_{1}^{n}, x_{2}^{n}\right] \cup\left[x_{2}^{n}, p_{n}\right] .
$$

Dado $\varepsilon>0$, seja $n_{0}=n(\varepsilon)$ como na definição de visibilidade Finsler. Então, para $n \geqslant n_{0}$, o ângulo de Landsberg entre $\left[p_{n}, x_{1}^{n}\right]$ e $\left[p_{n}, x_{2}^{n}\right]$ em $p_{n}$ é no máximo $\varepsilon$, pela visibilidade para $t \geqslant 0$. Ao mesmo tempo, o ângulo de Landsberg entre $\left[x_{0}^{n}, p_{n}\right]$ e $\left[x_{2}^{n}, p_{n}\right]$ em $p_{n}$ é no máximo $\varepsilon$, pela visibilidade para $t \leqslant 0$. Como o comprimento das geodésicas minimizantes $\left[x_{0}^{n}, x_{1}^{n}\right]$ cresce arbitrariamente com $n$ e o ângulo de Landsberg dessas geodésicas com $\left[p_{n}, x_{2}^{n}\right]$ e $\left[x_{2}^{n}, p_{n}\right]$ em $p_{n}$ tende a zero quando $n \rightarrow \infty$, existem $\ell_{n} \rightarrow \infty, y_{n} \in\left[x_{2}^{n}, p_{n}\right]$ e $q_{n} \in\left[p_{n}, x_{2}^{n}\right]$ tais que

1. A distância de todo ponto de $\left[y_{n}, p_{n}\right]$ a $\left[x_{0}^{n}, x_{1}^{n}\right]$ tende a zero quando $n \rightarrow \infty$;

2. A distância de todo ponto de $\left[p_{n}, q_{n}\right]$ a $\left[x_{0}^{n}, x_{1}^{n}\right]$ tende a zero quando $n \rightarrow \infty$;

3. Os comprimentos de $\left[y_{n}, p_{n}\right]$ e de $\left[p_{n}, q_{n}\right]$ são maiores que $\ell_{n}$.

Já que $\left[x_{0}^{n}, x_{1}^{n}\right]$ é minimizante, a desigualdade triangular nos dá que a distância de $y_{n} \in\left[x_{2}^{n}, p_{n}\right]$ a $q_{n} \in\left[p_{n}, x_{2}^{n}\right]$ é maior que $\ell_{n}$ para $n$ suficientemente grande, o que contradiz a Proposição 2.20.

\subsection{2}

\section{$\delta$-hiperbolicidade e divergência implicam visibilidade}

Lema 2.24. Seja $(M, F)$ uma variedade Finsler de classe $C^{\infty}$ sem pontos conjugados. Se $\left(\tilde{M}, d_{\tilde{F}}\right)$ é um espaço Gromov-Finsler $\delta$-hiperbólico e os raios geodésicos divergem uniformemente para $t \geqslant 0$ e para $t \leqslant 0$, então $(\tilde{M}, \tilde{F})$ é de visibilidade uniforme.

Demonstração. Sejam $\gamma, \beta: \mathbb{R} \longrightarrow \tilde{M}$ duas geodésicas tais que $\gamma(0)=\beta(0)=p$ e suponha que

$$
d_{\tilde{F}}(p,[\gamma(t), \beta(r)])>n \text {. }
$$

Como $\left(\tilde{M}, d_{\tilde{F}}\right)$ é Gromov-Finsler $\delta$-hiperbólico, existem $z \in[\gamma(t), \beta(r)], x \in$ $\gamma([0, t])=[\gamma(0), \gamma(t)]$ e $y \in \beta([0, r])=[\beta(0), \beta(r)]$ tais que 
1. $d_{\tilde{F}}(z, x) \leqslant \delta$ e $d_{\tilde{F}}(z, y) \leqslant \delta$

2. $d_{\tilde{F}}(x, y) \leqslant 2 \delta$.

Pela desigualdade triangular,

$$
d_{\tilde{F}}(p, x)+d_{\tilde{F}}(x, z) \geqslant d_{\tilde{F}}(p, z) \geqslant n
$$

e

$$
d_{\tilde{F}}(p, y)+d_{\tilde{F}}(y, z) \geqslant d_{\tilde{F}}(p, z) \geqslant n,
$$

o que implica

$$
d_{\tilde{F}}(p, x) \geqslant n-d_{\tilde{F}}(x, z) \geqslant n-C d_{\tilde{F}}(z, x) \geqslant n-C \delta
$$

$\mathrm{e}$

$$
d_{\tilde{F}}(p, y) \geqslant n-d_{\tilde{F}}(y, z) \geqslant n-C d_{\tilde{F}}(z, y) \geqslant n-C \delta
$$

Fixe $\varepsilon>0$ e $R=2 \delta$ na definição de divergência uniforme de raios geodésicos. As desigualdades $d_{\tilde{F}}(p, x) \geqslant n-C \delta$ e $d_{\tilde{F}}(p, y) \geqslant n-C \delta$ juntamente com $d_{\tilde{F}}(x, y)=d_{\tilde{F}}\left(\gamma\left(t_{x}\right), \beta\left(r_{y}\right)\right) \leqslant 2 \delta$ dizem que para $t_{x}, r_{y} \in[0, \infty)$ arbitrariamente grandes, ocorre

$$
\min \left\{d_{\tilde{F}}\left(\gamma\left(t_{x}\right), \beta\left(r_{y}\right)\right), d_{\tilde{F}}\left(\beta\left(r_{y}\right), \gamma\left(t_{x}\right)\right)\right\} \leqslant R
$$

Portanto, $\varangle_{p}\left(\gamma^{\prime}(0), \beta^{\prime}(0)\right) \leqslant \varepsilon$.

\section{6}

\section{Metrização de um grupo finitamente gerado}

Seja $(G, \cdot)$ um grupo finitamente gerado e $S=\left\{a_{1}, \ldots, a_{n}\right\}$ um conjunto simétrico de geradores (ou seja, $S=S^{-1}:=\left\{a^{-1} / a \in S\right\}$ e $G=\langle S\rangle$ ). Denote por $e$ o elemento neutro do grupo $(G, \cdot)$. Um elemento de $G$ é portanto uma palavra em $G$, ou seja, $g=a_{i_{1}}^{k_{1}} \cdots a_{i_{s}}^{k_{s}}$ onde $a_{i_{j}} \in S$ e $k_{j} \in \mathbb{N}$. Dado $g \in G$, sempre consideraremos que $g=a_{i_{1}}^{k_{1}} \cdots a_{i_{s}}^{k_{s}}$ é a palavra com menor número de fatores. O comprimento de $g$ é o número $\ell(g):=k_{1}+\cdots+k_{s}$. Convencionaremos que $\ell(e)=0$.

Definição 2.25. Seja $G$ um grupo finitamente gerado e $S=\left\{a_{1}, \ldots, a_{n}\right\}$ um gerador simétrico de $G$. A métrica da palavra em $G$ é a função $d: G \times G \longrightarrow \mathbb{R}$ definida por $d(a, b)=\ell\left(a \cdot b^{-1}\right)$. 
A função $d$ definida acima faz de $(G, d)$ um espaço métrico. Para um tratamento extenso e sistemático das propriedades de $(G, d)$, sugerimos por exemplo [8] e [10]. Em particular, fixado $g \in G$, a função $f: G \longrightarrow G$ dada por $f(a)=g \cdot a$ é uma isometria de $(G, d)$ e portanto a ação natural de $G$ em $G$ pela esquerda (e, analogamente, pela direita) é por isometrias da métrica da palavra. É claro que o comprimento de um elemento de $g$, e portanto a própria definição da métrica $d$, dependem da escolha do gerador $S$, mas se $d$ e $\bar{d}$ são duas métricas da palavra em $G$, então $(G, d)$ e $(G, \bar{d})$ são $(A, B)$-quaseisométricos para algum par $A, B \in \mathbb{R}$.

A métrica da palavra induz uma estrutura de espaço métrico geodésico no grafo de Cayley de $G$. Mais precisamente, seja $(G, \cdot)$ um grupo finitamente gerado e

$$
S=\left\{a_{1}, \ldots a_{k}, a_{1}^{-1}, \ldots, a_{k}^{-1}\right\}=\left\{a_{1}, \ldots a_{k}\right\} \cup\left\{a_{1}^{-1}, \ldots, a_{k}^{-1}\right\}=: S^{+} \cup S^{-}
$$

um gerador simétrico de $G$. O grafo de Cayley $\Gamma_{S}(G)$ de $G$ é construído da seguinte forma: para um ponto base $p_{0} \in S$ fixado, construa uma aresta ligando $p_{0}$ aos pontos do conjunto $A_{1}\left(p_{0}\right):=\left\{a \cdot p_{0} / a \in S\right\}$. Agora, para cada $j \in \mathbb{N}$, defina recursivamente os conjuntos

$$
A_{j+1}\left(p_{j}\right):=\left\{a \cdot p_{j} / p_{j} \in A_{j} \text { e } a \in S\right\}
$$

e construa uma aresta ligando $p_{j} \in A_{j}$ a cada elemento de $A_{j+1}$. Em outras palavras:

- Os vértices de $\Gamma_{S}(G)$ são os elementos de $G$.

- Existe uma aresta ligando $x \in G$ a $y \in G$ se e somente se existe $a \in S$ tal que $y=a \cdot x$.

As arestas são 1-células homeomorfas a $[0,1] \subset \mathbb{R}$ e, neste sentido, com a topologia natural desses intervalos, o grafo assim construído é um espaço topológico conexo por caminhos. Assim, a métrica da palavra de $G$ se estende a todos os pontos de $\Gamma_{S}(G)$, fazendo de $\left(\Gamma_{S}(G), d\right)$ um espaço métrico geodésico completo, onde $d$ denota a métrica da palavra estendida.

\subsection{1}

\section{Compactificação de $\Gamma_{S}(G)$}

Revisitamos brevemente as noções usuais de $\delta$-hiperbolicidade de Gromov introduzidas em [8] (compare com a Definição 2.9). 
Definição 2.26. Seja $\delta>0$ fixado. Um espaço métrico geodésico completo $(X, d)$ é Gromov $\delta$-hiperbólico se para todo $x_{0}, x_{1}, x_{2} \in X$ e para todo segmento geodésico $\left[x_{1}, x_{j}\right], j=1,2,3$, vale

$$
d\left(\left[x_{i}, x_{j}\right] \cup\left[x_{j}, x_{k}\right], x\right) \leqslant \delta
$$

para todo $x \in\left[x_{i}, x_{k}\right]$.

A definição de espaço métrico Gromov $\delta$-hiperbólico acima será especializada para tratarmos do grafo de Cayley de um grupo finitamente gerado.

Definição 2.27. Um grupo finitamente gerado $G$ é Gromov $\delta$-hiperbólico se $\left(\Gamma_{S}(G), d\right)$ com a métrica da palavra é um espaço métrico Gromov $\delta$-hiperbólico para algum (e portanto para todo) gerador finito simétrico $S$ e para algum $\delta>0$.

Observe que $\left(\Gamma_{S}(G), d\right)$ é um espaço métrico Finsler, completo e geodésico no qual a métrica satisfaz $d(x, y)=d(y, x)$. Portanto, as noções de f-assintoticidade e b-assintoticidade (Definição 2.5) coincidem, assim como as de convergencia dadas pelas Definições 2.6 e 2.7. Em particular,

$$
\partial \Gamma_{S}(G)(+\infty)=\partial \Gamma_{S}(G)(-\infty):=\partial \Gamma_{S}(G) .
$$

Proposição 2.28. Seja $G$ um grupo Gromov $\delta$-hiperbólico. Então $\overline{\Gamma_{S}(G)} m u$ nido da noção de convergência dada pela Definição 2.6 é um espaço topológico compacto, no qual $B \subset \overline{\Gamma_{S}(G)}$ é fechado se e somente se

$$
x_{n} \in B \text { e } x_{n} \rightarrow x \quad \text { implica } \quad x \in B
$$

Além disso,

1. A topologia assim gerada independe da escolha do ponto $p$.

2. A inclusão $X \subset X(+\infty)$ é um homeomorfismo sobre $\Gamma_{S}(G)$.

3. $\partial \Gamma_{S}(G)$ é compacto.

Demonstração. Novamente, referimos ao leitor [14], Proposition 3.7.

A ação de $G$ em $G$ à esquerda induz uma ação de $G$ em $\Gamma_{S}(G)$ por isometrias da métrica da palavra. Os resultados abaixo, devidos a Gromov [8], nos fornecem uma importante descrição desta ação.

Se $G$ é um grupo Gromov $\delta$-hiperbólico e $\gamma \in G$ é um elemento hiperbólico (ou seja, o mapa $\mathbb{Z} \longrightarrow \Gamma_{S}(G)$ dado por $i \longmapsto \gamma^{i} \cdot x$ para algum - e portanto 
para todo $-x \in \Gamma_{S}(G)$ fixado é uma quase-isometria), os limites

$$
\gamma^{-\infty}:=\lim _{i \rightarrow-\infty} \gamma^{i} \in \partial \Gamma_{S}(G) \quad \text { e } \quad \gamma^{+\infty}:=\lim _{i \rightarrow+\infty} \gamma^{i} \in \partial \Gamma_{S}(G)
$$

existem e são distintos. Além disso,

Teorema 2.29 (Gromov [8], Corollary 8.2.G). O conjunto de pares $\left(\gamma^{-\infty}, \gamma^{+\infty}\right) \in \partial \Gamma_{S}(G) \times \partial \Gamma_{S}(G)$ para todo $\gamma \in G$ hiperbólico é denso em $\partial \Gamma_{S}(G) \times \partial \Gamma_{S}(G)$.

Observe que se $\left(\Gamma_{S}(G), d\right)$ é Gromov $\delta$-hiperbólico, então cada isometria da ação de $G$ em $\Gamma_{S}(G)$ se estende a um homeomorfismo de $\overline{\Gamma_{S}(G)}$ (Proposição 2.14). Em particular,

Teorema $2.30(-, 8.2 . \mathrm{H})$. A ação de $G$ em $\partial \Gamma_{S}(G) \times \partial \Gamma_{S}(G)$ é topologicamente transitiva. Ou seja, todo aberto não-vazio $G$-invariante é denso.

Teorema 2.31 (-, 8.2.I). Existe um ponto em $\partial \Gamma_{S}(G) \times \partial \Gamma_{S}(G)$ cuja G-órbita é densa. 


\section{3}

\section{Visibilidade e a transitividade do fluxo geodésico}

O conjunto de hipóteses que adotaremos daqui até o final do capítulo será, salvo menção contrária explícita:

- $(M, F)$ é uma variedade Finsler compacta de dimensão $n$;

- $(M, F)$ não tem pontos conjugados;

- $(\tilde{M}, \tilde{F})$ é uma variedade de visibilidade uniforme.

Nosso objetivo é apresentar a prova do seguinte resultado, que constitui uma de nossas duas principais contribuições à atual e extensa investigação acerca de variedades Finsler:

Teorema A. Seja $(M, F)$ uma variedade Finsler compacta $C^{\infty}$, sem pontos conjugados e de visibilidade uniforme. Então seu fluxo geodésico $\varphi_{t}: \mathcal{S} M \longrightarrow$ $\mathcal{S} M$ é transitivo.

Este capítulo está assim estruturado: primeiramente, construiremos topologias em $\tilde{M}(-\infty)$ e $\tilde{M}(+\infty)$ análogas à topologia de cones introduzida por Eberlein em [7], levando em conta a eventual falta de reversibilidade das geodésicas (seção 3.1). Mostraremos que as noções de convergência para estas topologias coincidem com as dadas pelas Definições 2.6 e 2.7. Usando isso, provaremos que as compactificações $\tilde{M}(-\infty)$ e $\tilde{M}(+\infty)$ são naturalmente homeomorfas (seção 3.2).

Em seguida (seção 3.3), descreveremos algumas propriedades da ação do grupo fundamental de $\tilde{M}$ em $\tilde{M}(-\infty)$ e $\tilde{M}(+\infty)$ e sua relação com o homeomorfismo natural entre elas.

Após isso (seção 3.4), construiremos explicitamente uma quase-isometria entre $\Gamma_{S}\left(\pi_{1}(M)\right)$ e $\tilde{M}$. Com ela, concluiremos que a ação de $\pi_{1}(M)$ em $\partial \tilde{M}(+\infty) \times \partial \tilde{M}(+\infty)$ tem órbita densa (Corolário 3.16).

O próximo passo (seção 3.5) será definir, no contexto Finsler, o análogo da noção de dualidade descrita por Eberlein em [7]. Para isso, usaremos o homeomorfismo entre as compactificações de $\tilde{M}$. A dualidade é a noção que relaciona a dinâmica do grupo a um parâmetro de difeomorfismos descrito pelo fluxo geodésico com a ação discreta do grupo fundamental de $M$ em $\tilde{M}$. A 
órbita densa obtida no Corolário 3.16 será a chave para a demonstração de que todos os pontos do bordo de $\tilde{M}$ são duais (subseção 3.5.2), uma propriedade central para obtermos a transitividade do fluxo geodésico propriamente dita (seção 3.6).

\section{1}

\section{As topologias de cones}

Vamos munir os conjuntos $\tilde{M}(+\infty)$ e $\tilde{M}(-\infty)$ de topologias análogas às topologias de cones em variedades Riemannianas de visibilidade, seguindo as ideias contidas em [7]. Usaremos o seguinte resultado adiante:

Lema 3.1. Dados $p \in \tilde{M}$ e $\gamma(-\infty) \in \partial \tilde{M}(-\infty)$, existem $R>0$ e uma única geodésica $\sigma: \mathbb{R} \longrightarrow \tilde{M}$ tal que $\sigma(0)=p$ e $d_{F}(\gamma(t), \sigma(t))<R$ para todo $t<0$.

Demonstração. Seja $t_{n} \rightarrow-\infty$ e considere geodésicas unitárias $\sigma_{n}$ : $\left[-a_{n}, 0\right] \longrightarrow \tilde{M}$ tais que $\sigma\left(-a_{n}\right)=\gamma\left(-t_{n}\right)$ e $\sigma(0)=p$, onde $a_{n}=d_{F}\left(\gamma\left(-t_{n}\right), p\right)$. Temos que $\sigma_{n}^{\prime}(0) \in \mathcal{S}_{p}$ e portanto, a menos de considerarmos uma subsequência, existe $v \in \mathcal{S}_{p}$ tal que $\sigma_{n}^{\prime}(0) \rightarrow v$. Afirmamos que a geodésica $\sigma$ tal que $\sigma(0)=p$ e $\sigma^{\prime}(0)=v$ satisfaz ao enunciado do Lema. De fato, como $\tilde{M}$ é Gromov-Finsler $\delta$-hiperbólico, os triâgulos geodésicos de arestas $\left[\gamma\left(-t_{n}\right), p\right]$, $\left[\gamma\left(-t_{n}\right), \gamma(0)\right]$ e $[\gamma(0), p]$ satisfazem aos axiomas de $\delta$-hiperbolicidade. Em particular, como o comprimento do segmento $[\gamma(0), p]$ independe de $n$ e $d_{F}\left(\sigma\left(-t_{n}\right), p\right) \rightarrow \infty$, temos que para $n$ suficientemente grande os pontos $\sigma\left(-t_{n}\right)$ satisfazem $d_{F}\left(\sigma\left(-t_{n}\right), \gamma\right) \leqslant \delta$. Portanto, $\sigma$ e $\gamma$ são b-assintóticas.

Naturalmente, o mesmo vale para geodésicas f-assintóticas:

Lema 3.2. Dados $p \in \tilde{M}$ e $\gamma(+\infty) \in \partial \tilde{M}(+\infty)$, existem $R>0$ e uma única geodésica $\sigma: \mathbb{R} \longrightarrow \tilde{M}$ tal que $\sigma(0)=p$ e $d_{F}(\gamma(t), \sigma(t))<R$ para todo $t>0$.

Lembremos que na ausência de pontos conjugados, $\exp _{p}: T_{p} \tilde{M} \longrightarrow \tilde{M}$ é um difeomorfismo para todo $p \in \tilde{M}$. Em particular, dados $p, q \in \tilde{M}$, existe uma única geodésica unitária $\gamma: \mathbb{R} \longrightarrow \tilde{M}$ tal que $\gamma(0)=p$ e $\gamma(\ell)=q$, onde $\ell=d_{\tilde{F}}(p, q)$. Sejam então $p, q, r \in \tilde{M}$. Defina

$$
\varangle_{p}^{+}(q, r):=\varangle_{p}(v, w)
$$

onde $v$ e $w$ são respectivamente os vetores tangentes em $p$ às geodésicas unitárias $[p, q]$ e $[p, r]$ (o símbolo "+" tem a função de evidenciar que estamos considerando raios que partem de $p$ ). Com o Lema 3.2, podemos estender a noção de ângulo de Landsberg para pontos de $\partial \tilde{M}(+\infty)$. Mais precisamente, 
sejam $p, q \in \tilde{M}$ e $\gamma: \mathbb{R} \rightarrow \tilde{M}$ a única geodésica tal que $\gamma(+\infty) \in \partial M(+\infty)$, $\gamma(0)=p$ e $q \in \tilde{M}$. Definimos

$$
\varangle_{p}^{+}(\gamma(+\infty), q):=\varangle_{p}\left(\gamma^{\prime}(0), w\right),
$$

onde $w$ é o vetor tangente em $p$ à geodésica unitária $[p, q]$.

Para construir uma topologia em $\tilde{M}(+\infty)$, considere um ponto $p \in \tilde{M}$. Uma base de abertos de $p$ será simplesmente uma base de abertos da topologia da variedade diferenciável $\tilde{M}$. Para cada ponto $\gamma(+\infty) \in \partial \tilde{M}(+\infty)$ e para cada $\varepsilon>0$, o f-cone de vértice $p$, ângulo $\varepsilon$ e eixo $\gamma(+\infty)$ é o conjunto

$$
C_{\gamma, \varepsilon}^{+}(p):=\left\{q \in \tilde{M}(+\infty) / \varangle_{p}^{+}(\gamma(+\infty), q)<\varepsilon\right\} .
$$

Um $f$-cone truncado de vértice $p$, ângulo $\varepsilon>0$ e raio $r>0$ em torno de $\gamma(+\infty)$ é o conjunto

$$
C_{\gamma, \varepsilon, r}^{+}(p):=\left\{q \in \tilde{M}(+\infty) / \varangle_{p}^{+}(\gamma(+\infty), q)<\varepsilon \text { e } d_{F}(p, q)>r\right\}
$$

O resultado a seguir é clássico para o recobrimento universal de uma variedade Riemanniana sem pontos conjugados e de visibilidade (ver por exemplo [7]). A prova da versão Finsler é uma extensão natural do caso Riemanniano.

Teorema 3.3. Os abertos de $\tilde{M}$ juntamente com os f-cones truncados formam uma topologia para $\tilde{M}(+\infty)$, com a qual $\tilde{M}(+\infty)$ é um espaço topológico compacto e $\partial \tilde{M}(+\infty)$ é homeomorfo a $\mathbb{S}^{n-1}$, onde $n=\operatorname{dim}(M)$.

Para definir uma topologia semelhante à de cones no conjunto $\tilde{M}(-\infty)$, basta usarmos o Lema 3.1, que permite estabelecer a noção de ângulo

$$
\varangle_{p}^{-}(\gamma(-\infty), q):=\varangle_{p}\left(\gamma^{\prime}(0), w\right),
$$

onde estamos tomando $p, q \in \tilde{M}, w$ é o vetor tangente em $p$ à geodésica unitária $[q, p]$ e $\gamma: \mathbb{R} \rightarrow \tilde{M}$ a única geodésica tal que $\gamma(0)=p$ e $\gamma(-\infty) \in \partial M(-\infty)$. $\mathrm{O} b$-cone de vértice $p$, ângulo $\varepsilon$ e eixo $\gamma(-\infty)$ é o conjunto

$$
C_{\gamma, \varepsilon}^{-}(p):=\left\{q \in \tilde{M}(-\infty) / \varangle_{p}^{-}(\gamma(+\infty), q)<\varepsilon\right\}
$$

$\mathrm{Um}$ b-cone truncado de vértice $p$, ângulo $\varepsilon>0$ e raio $r>0$ em torno de $\gamma(-\infty)$ é o conjunto

$$
C_{\gamma, \varepsilon, r}^{-}(p):=\left\{q \in \tilde{M}(-\infty) / \varangle_{p}^{-}(\gamma(-\infty), q)<\varepsilon \text { e } d_{F}(q, p)>r\right\} .
$$


Analogamente à topologia de f-cones, na topologia de b-cones temos o seguinte

Teorema 3.4. Os abertos de $\tilde{M}$ juntamete com os b-cones truncados formam uma topologia para $\tilde{M}(-\infty)$, com a qual $\tilde{M}(-\infty)$ é um espaço topológico compacto e $\partial \tilde{M}(-\infty)$ é homeomorfo a $\mathbb{S}^{n-1}$, onde $n=\operatorname{dim}(M)$.

\subsection{1}

\section{Equivalência entre topologias}

Aqui enunciamos que as topologias definidas pelos Teoremas 3.3 e 3.4 acima coincidem com aquelas dadas pelas Definições 2.6 e 2.7. Este resultado permitirá transferir (via um homeomorfismo oriundo de uma quase-isometria - ver seção 3.2) propriedades dinâmicas da ação de $\pi_{1}(M)$ em $\partial \Gamma_{S}\left(\pi_{1}(M)\right)$ para $\partial \tilde{M}(+\infty)$ (subseção 3.4.2, Corolário 3.16).

Lema 3.5. Seja $(M, F)$ uma variedade Finsler compacta, sem pontos conjugados e tal que $(\tilde{M}, \tilde{F})$ é de visibilidade uniforme. Então, em $\tilde{M}(+\infty)$ a noção de convergência dada pela Definição 2.6 é equivalente à convergência na topologia de f-cones.

Demonstração. Primeiramente, observe que os abertos, fechados e compactos de $\tilde{M}$ coincidem em ambas as topologias e são os mesmos da topologia de $\tilde{M}$ como variedade. Portanto, basta verificar o lema para pontos limite no bordo de sequências de $\tilde{M}(+\infty)$.

Seja $\left\{x_{n}\right\} \subset \tilde{M}(+\infty)$ com $x_{n} \rightarrow x \in \partial \tilde{M}(+\infty)$ na topologia de f-cones e fixe $p \in \tilde{M}$. Denote por $\gamma$ a única geodésica tal que $\gamma(0)=p$ e $\gamma(+\infty)=x$. Dado $\varepsilon>0$, existe $n_{\varepsilon}$ tal que para $n \geqslant n_{\varepsilon}$ vale $\left(\left[p, x_{n}\right]-\{p\}\right) \subset C_{\gamma, \varepsilon, r}(p)$ para $r$ fixado. Denotando por $\gamma_{n}$ as geodésicas que ligam $p$ a $x_{n}$, temos em particular que $\varangle_{p}\left(\gamma^{\prime}(0), \gamma_{n}^{\prime}(0)\right) \rightarrow 0$ quando $j \rightarrow \infty$, o que implica $\gamma_{n} \rightarrow \gamma$ em compactos.

Agora suponha que $x_{n} \rightarrow x$ segundo a Definição 2.6. Tome novamente $\gamma_{n}, \gamma: \mathbb{R} \longrightarrow \tilde{M}(+\infty)$ geodésicas unitárias tais que $\gamma_{n}(0)=\gamma(0)=p$ e $\gamma_{n}(+\infty)=x_{n}$. Em particular, $\varangle_{p}\left(\gamma^{\prime}(0), \gamma_{n}^{\prime}(0)\right) \rightarrow 0$ pela convergência de $\gamma_{n}$ no compacto $B_{r}^{+}(p)$, para qualquer $r>0$ fixado. Se existissem $\varepsilon>0, r^{\prime}>0$ tais que alguma subsequência $\gamma_{n_{j}}\left(t_{n_{j}}\right)$ satisfizesse $\gamma_{n_{j}}\left(t_{n_{j}}\right) \notin C_{\gamma, \varepsilon, r^{\prime}}(p)$, então

$$
\lim _{j \rightarrow \infty} \varangle_{p}\left(\gamma^{\prime}(0), \gamma_{n_{j}}^{\prime}(0)\right)=\lim _{j \rightarrow \infty} \varangle_{p}^{+}\left(\gamma(+\infty), \gamma_{n_{j}}\left(t_{n_{j}}\right)\right) \geqslant \varepsilon,
$$

uma contradição.

O caso da topologia de b-cones e da noção de convergência da Definição 2.7 é análogo. 


\section{2}

\section{Um homeomorfismo natural entre $\partial \tilde{M}(-\infty)$ e $\partial \tilde{M}(+\infty)$}

Construiremos nesta subseção um homeomorfismo natural entre as compactificações de $\tilde{M}$. Este homeomorfismo desempenhará um importante papel na definição do conceito de dualidade (Definição 3.17) logo adiante.

Lema 3.6. Existe uma bijeção natural $h: \tilde{M}(-\infty) \longrightarrow \tilde{M}(+\infty)$.

Demonstração. Sejam $\gamma(-\infty) \in \partial \tilde{M}(-\infty), s_{n} \in \mathbb{R}$ e $t_{n} \in \mathbb{R}$ sequências tais que $s_{n} \rightarrow-\infty$ e $t_{n} \rightarrow \infty$. Considere a sequência de segmentos geodésicos minimizantes, unitários e orientados $\beta_{n}:\left[s_{n}, t_{n}\right] \longrightarrow \tilde{M}$ tal que $\beta\left(t_{n}\right)=\gamma\left(s_{n}\right)$ e $\beta\left(s_{n}\right)=\gamma\left(t_{n}\right)$. Pela Proposição 2.20, existe $A>0$ tal que, para todo $n, \beta_{n}$ está a uma distância no máximo $A$ de $\gamma$. Portanto, a menos de considerar uma subsequência, existe uma geodésica $\beta: \mathbb{R} \longrightarrow M$ tal que $\beta_{n}$ converge para $\beta$ uniformemente em compactos da topologia métrica associada a $\bar{d}$ (ver (2-1)). Considere a aplicação

$$
h: \tilde{M}(-\infty) \longrightarrow \tilde{M}(+\infty)
$$

dada por $h(x)=x$ se $x \in \tilde{M}$ e $h(\gamma(-\infty))=\beta(+\infty)$.

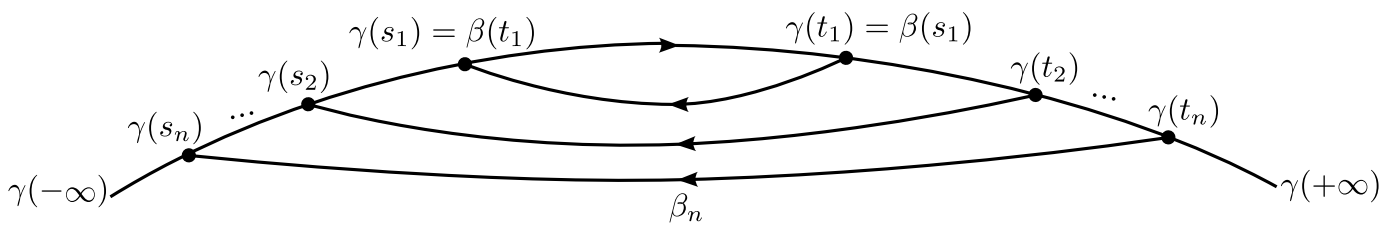

Figura 3.1: Construindo a função $h$ em $\partial \tilde{M}(-\infty)$.

Vejamos que essa aplicação está bem definida no bordo, ou seja:

- independe de $\beta$ : de fato, novamente pela Proposição 2.20, qualquer sequência $\bar{\beta}_{n}$ de segmentos de geodésicas com pontos iniciais e finais sobre $\gamma$ estará no máximo a uma distância $A>0$ de $\gamma \cdot \operatorname{Logo}, \lim \bar{\beta}_{n}=\bar{\beta}$ é uma geodésica f-assintótica a $\beta$. Portanto, $\beta(+\infty)=\bar{\beta}(+\infty)$.

- independe da escolha de geodésida em $\gamma(-\infty)$ : tome uma geodésica $\eta \in \gamma(-\infty)$ e uma sequência $\sigma_{n}$ de geodésicas - com pontos inicias e finais sobre $\eta$, como anteriormente - tal que $\lim \sigma_{n}=h(\eta(-\infty))=\sigma(+\infty)$. Então, $\sigma$ está a uma distância no máximo $A$ de $\eta$. Como $\gamma$ e $\eta$ são fassintóticas e $\beta$ está a uma distância no máximo $A$ de $\gamma$, então $\beta$ e $\sigma$ são b-assintóticas, ou seja,

$$
\beta(+\infty)=h(\gamma(-\infty))=h(\eta(-\infty))=\sigma(+\infty) .
$$

Concluímos que $h$ está bem definida. 
Vejamos que $h$ é injetiva. Sejam $\gamma(-\infty), \bar{\gamma}(-\infty) \in \partial \tilde{M}(-\infty)$ e suponha que

$$
\beta(+\infty)=h(\gamma(-\infty))=h(\bar{\gamma}(-\infty))=\bar{\beta}(+\infty) .
$$

Então $\beta$ e $\bar{\beta}$ são f-assintóticas. Como $\beta$ e $\bar{\beta}$ estão a uma distância no máximo $A$ de $\gamma$ e $\bar{\gamma}$ respectivamente, temos pela desigualdade triangular que $\gamma$ e $\bar{\gamma}$ são b-assintóticas, donde $\gamma(-\infty)=\bar{\gamma}(-\infty)$.

Vejamos finalmente que $h$ é sobrejetiva. Seja $\beta(+\infty) \in \partial \tilde{M}(+\infty)$. Construa uma sequência de geodésicas minimizantes $\gamma_{n}:\left[s_{n}, t_{n}\right] \longrightarrow M \mathrm{com}$ $s_{n} \longrightarrow-\infty, t_{n} \longrightarrow \infty$ e $\gamma\left(s_{n}\right)=\beta\left(t_{n}\right)$ e $\gamma\left(t_{n}\right)=\beta\left(s_{n}\right)$. Pela Proposição 2.20, $\gamma_{n}$ está a uma distância no máximo $A>0$ de $\beta$ e o limite $\gamma_{n} \longrightarrow \gamma$ é uma geodésica tal que $h(\gamma(-\infty))=\beta(+\infty)$.

Para demonstrar que a função $h$ acima construída é um homeomorfismo, precisaremos de algumas definições e resultados auxiliares. Sejam $p, q \in M$, $[p, q]$ a geodésica unitária orientada ligando $p$ e $q$. Consideraremos as funções

$$
V^{+}: \tilde{M} \times \tilde{M} \longrightarrow \mathcal{S} \tilde{M} \quad \text { e } \quad V^{-}: \tilde{M} \times \tilde{M} \longrightarrow \mathcal{S} \tilde{M}
$$

definidas da seguinte forma: $V^{+}(p, q)$ é o vetor tangente a $[p, q]$ em $p$ e $V^{-}(p, q)$ é o vetor tangente a $[p, q]$ em $q$.

Lema 3.7. Sejam $p_{n}, q_{n}, x_{n}$ sequências em $\tilde{M}$ tais que $p_{n} \rightarrow p, q_{n} \rightarrow q$ e sejam $\beta, \sigma: \mathbb{R} \longrightarrow \tilde{M}$ geodésicas com $\beta(0)=p, \beta^{\prime}(0)=v, \sigma(0)=q$ e $\sigma^{\prime}(0)=w$.

1. Se $x_{n} \rightarrow x \in \partial \tilde{M}(-\infty), V^{-}\left(x_{n}, p_{n}\right) \rightarrow v$ e $V^{-}\left(x_{n}, q_{n}\right) \rightarrow w$, então $\beta$ e $\sigma$ são b-assintóticas.

2. Se $x_{n} \rightarrow x \in \partial \tilde{M}(+\infty), V^{+}\left(p_{n}, x_{n}\right) \rightarrow v$ e $V^{+}\left(q_{n}, x_{n}\right) \rightarrow w$, então $\beta$ e $\sigma$ são f-assintóticas.

Demonstração. Vamos esboçar a prova do item 1. As geodésicas $\left[x_{n}, p_{n}\right]$ convergem em compactos para o raio geodésico ligando $x$ a $p$, assim como as geodésicas $\left[x_{n}, q_{n}\right]$ convergem para o único (pelo Lema 3.1 ) raio geodésico ligando $x$ a $q$. Tais raios são portanto b-assintóticos. Mas pela unicidade no Lema 3.1, tais raios coincidem com $\beta$ e $\sigma$ respectivamente.

Podemos estender as definições de $V^{+}$e de $V^{-}$aos bordos e considerar

$$
V^{+}: \tilde{M} \times \tilde{M}(+\infty) \longrightarrow \mathcal{S} \tilde{M} \quad \text { e } \quad V^{-}: \tilde{M}(-\infty) \times \tilde{M} \longrightarrow \mathcal{S} \tilde{M}
$$

de maneira natural: dados $\gamma(-\infty) \in \partial \tilde{M}(-\infty)$ e $p \in \tilde{M}$, basta defirnimos $V^{-}(\gamma(-\infty), p)=\sigma^{\prime}(0)$, onde $\sigma$ é a única geodésica unitária b-assintótica a $\gamma$ 
com $\sigma(0)=p$. Analogamente, se $p \in \tilde{M}$ e $\gamma(+\infty) \in \partial \tilde{M}(+\infty)$, definimos $V^{+}(p, \gamma(+\infty))=\sigma^{\prime}(0)$, onde $\sigma$ é a única geodésica f-assintótica a $\gamma$ com $\sigma(0)=p$.

As seguintes propriedade das funções $V^{+}$e $V^{-}$são relativamente simples de verificar usando a divergência para $t<0$ e $t>0$ de raios geodésicos com um ponto em comum.

Lema 3.8. Seja $p \in \tilde{M}$ e $\gamma: \mathbb{R} \longrightarrow \tilde{M}$ uma geodésica. Então

1. $V^{-}(\gamma(t), p) \rightarrow V^{-}(\gamma(-\infty), p)$ quando $t \rightarrow-\infty$,

2. $V^{+}(p, \gamma(t)) \rightarrow V^{+}(p, \gamma(+\infty))$ quando $t \rightarrow+\infty$.

Seja $\theta=(p, v) \in \mathcal{S} \tilde{M}$. Denotaremos por $\gamma_{\theta}: \mathbb{R} \longrightarrow \tilde{M}$ a única geodésica tal que $\gamma_{\theta}(0)=p$ e $\gamma_{\theta}^{\prime}(0)=v$.

Lema 3.9. Seja $(\tilde{M}, \tilde{F})$ o recobrimento de uma variedade Finsler compacta sem pontos conjugados e de visibilidade uniforme. Então:

1. As funções $V^{+}: \tilde{M} \times \tilde{M}(+\infty) \longrightarrow \mathcal{S} \tilde{M}$ e $V^{-}: \tilde{M}(-\infty) \times \tilde{M} \longrightarrow \mathcal{S} \tilde{M}$ são contínuas no produto das topologias de cones correspondentes.

2. As funções que associam $\theta=(p, v)$ às classes assintóticas $\gamma_{\theta}(-\infty) e$ $\gamma_{\theta}(+\infty)$ são contínuas nas topologias de cones.

Enfim, provamos que a função $h$ é um homeomorfismo.

Proposição 3.10. Seja $(M, F)$ uma variedade Finsler compacta, sem pontos conjugados e de visibilidade. Então

$$
h: \tilde{M}(-\infty) \longrightarrow \tilde{M}(+\infty) \quad e \quad h: \partial \tilde{M}(-\infty) \longrightarrow \partial \tilde{M}(+\infty)
$$

são homeomorfismos.

Demonstração. Basta analisarmos o caso em que $x=\beta(-\infty)$ e $y=\gamma(+\infty)$ com $h(x)=y$. Considere uma sequência $\left\{x_{n}\right\} \subset \tilde{M}(-\infty)$ tal que $x_{n} \rightarrow x$. Podemos supor que $x_{n} \in \partial \tilde{M}(-\infty)$. Denote $p=\beta(0)$. Tome a sequência de geodésicas $\left[x_{n}, p\right]$, que converge em compactos para $[x, p]$ por hipótese. Afirmamos que a sequência $\left[p, h\left(x_{n}\right)\right]$ converge em compactos para $[p, h(x)]$. De fato, se alguma subsequência $\left[p, h\left(x_{n_{j}}\right)\right]$ não convergir em compactos para $[p, h(x)]$, então

$$
v:=\lim _{n \rightarrow \infty} V^{+}\left(p, h\left(x_{n}\right)\right) \neq V^{+}(p, h(x))
$$

e as geodésicas partindo de $p$ com direção $v$ e $V^{+}(p, h(x))$ divergem para $t>0$. Mas

$$
d_{\tilde{F}}\left(\left[x_{n}, p\right],\left[p, h\left(x_{n}\right)\right]\right) \leqslant A+2 C d_{\tilde{F}}(p, q)
$$


pela desigualdade triangular e pela Proposição 2.20. Então a geodésica limite da sequência $\left[x_{n}, p\right]$ diverge para $t<0$ da geodésica $[x, p]$, contradizendo a convergência $x_{n} \rightarrow x$.

No caso Riemanniano, a involução $\gamma_{(p, v)} \longmapsto \gamma_{(p,-v)}$ fornece imediatamente o homeomorfismo $h$ entre $\tilde{M}(+\infty)$ e $\tilde{M}(-\infty)$ : basta considerar $h\left(\gamma_{(p, v)}(+\infty)\right)=\gamma_{(p,-v)}(-\infty)$, pela reversibilidade da métrica - ou seja, a aplicação $h$ é a identidade (apenas consideramos uma classe assintótica como "futura" ou "passada", pois são a mesma). As topologias de f-cones e b-cones neste caso são coincidentes. No caso Finsler, a construção do homeomorfismo não é direta (pela falta de reversibilidade de $d_{\tilde{F}}$ ), mas como vimos acima é garantida pela Proposição 2.20.

\section{3}

\section{A ação de $\pi_{1}(M)$ em $\partial \tilde{M}(-\infty)$ e em $\partial \tilde{M}(+\infty)$}

Seja $\pi_{1}(M)$ o grupo fundamental de uma variedade Finsler compacta $(M, F)$, sem pontos conjugados tal que $(\tilde{M}, \tilde{F})$ é de visibilidade uniforme. Sabemos que a ação do grupo de isometrias $\pi_{1}(M)$ em $\tilde{M}$ é própria e descontínua. Vamos estender a ação de $\pi_{1}(M)$ aos espaços topológicos compactos $\tilde{M}(-\infty)$ e $\tilde{M}(+\infty)$. Se $\gamma(-\infty) \in \partial M(-\infty)$ e $\phi \in \pi_{1}(M)$, defina

$$
\phi(\gamma(-\infty)):=(\phi \circ \gamma)(-\infty)
$$

e analogamente, para $\beta \in \partial M(+\infty)$ e $\psi \in \pi_{1}(M)$, defina

$$
\psi(\beta(+\infty)):=(\psi \circ \beta)(+\infty) .
$$

Portanto, dada $\psi \in \pi_{1}(M)$, ficam definidas extensões

$$
\psi: \tilde{M}(-\infty) \longrightarrow \tilde{M}(-\infty) \quad \text { e } \quad \psi: \tilde{M}(+\infty) \longrightarrow \tilde{M}(+\infty)
$$

contínuas nas respectivas topologias de cones. Como a ação de $\pi_{1}(M)$ em $\tilde{M}$ é descontínua, toda $\psi \in \pi_{1}(M)$ com $\psi \neq$ Id não tem pontos fixos em $\tilde{M}$. Mas já que $\partial \tilde{M}(-\infty)$ e $\partial \tilde{M}(+\infty)$ são homeomorfos a esferas de dimensão $n-1$ (ver por exemplo [6], Proposition 1.13 para o caso Riemanniano), $\psi: \tilde{M}(-\infty) \longrightarrow \tilde{M}(-\infty)$ e $\psi: \tilde{M}(+\infty) \longrightarrow \tilde{M}(+\infty)$ têm pontos fixos em $\partial \tilde{M}(-\infty)$ e em $\partial \tilde{M}(+\infty)$ respectivamente, pelo teorema do ponto fixo de Brouwer. Na próxima seção estudaremos sistematicamente tais pontos fixos.

Observe que $h$ é equivariante com respeito às ações do grupo $\pi_{1}(M)$ em $\partial \tilde{M}(-\infty)$ e em $\partial \tilde{M}(+\infty)$, ou seja, os diagramas 

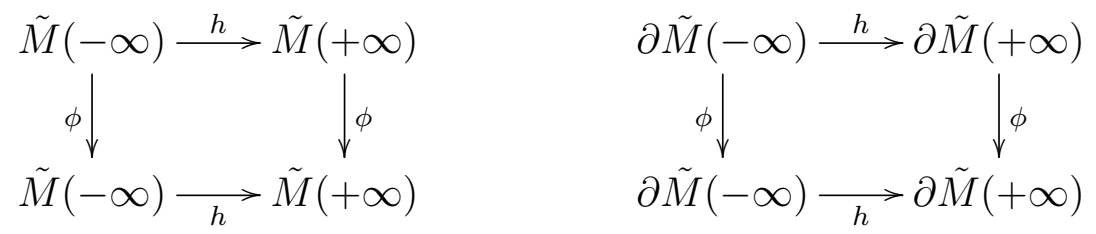

comutam para toda $\phi \in \pi_{1}(M)$. Este fato segue diretamente das definições de $h$ e das extensões de $\phi \in \pi_{1}(M)$ aos bordos $\partial \tilde{M}(+\infty)$ e $\partial \tilde{M}(-\infty)$. Portanto,

Corolário 3.11. Seja $\phi \in \pi_{1}(M)$ uma isometria. Então $x \in \partial \tilde{M}(-\infty)$ é ponto fixo de $\phi: \partial \tilde{M}(-\infty) \longrightarrow \partial \tilde{M}(-\infty)$ se e somente se $h(x)$ é ponto fixo de $\phi: \partial \tilde{M}(+\infty) \longrightarrow \partial \tilde{M}(+\infty)$.

\subsection{1}

\section{Geodésicas ligam pontos no infinito}

Provaremos a seguir que os pontos de $\partial \tilde{M}(-\infty)$ e de $\partial \tilde{M}(+\infty)$ não relacionados por $h$ estão ligados por pelo menos uma geodésica.

Proposição 3.12. Sejam $x \in \partial \tilde{M}(-\infty)$ e $y \in \partial \tilde{M}(+\infty)$ tais que $h(x) \neq y$. Então existe uma geodésica $\gamma: \mathbb{R} \longrightarrow \tilde{M}$ tal que $\gamma(-\infty)=x$ e $\gamma(+\infty)=y$.

Demonstração. Fixe $p \in \tilde{M}$ e sejam $\beta, \sigma: \mathbb{R} \longrightarrow \tilde{M}$ geodésicas unitárias satisfazendo

$$
\beta(0)=\sigma(0)=p \quad \beta(-\infty)=x \quad \sigma(+\infty)=y
$$

Se ocorrer $\beta^{\prime}(0)=\sigma^{\prime}(0)$, então as geodésicas $\beta$ e $\sigma$ são de fato a mesma e portanto $\beta(-\infty)=x$ e $\beta(+\infty)=y$. Suponhamos então que $\varangle_{p}\left(\beta^{\prime}(0), \sigma^{\prime}(0)\right)>0$. Tome $t_{n} \rightarrow \infty$. A menos de considerarmos uma subsequência, os segmentos geodésicos orientados ligando $p$ a $\beta\left(-t_{n}\right)$ convergem (uniformemente em compactos) para uma geodésica $\bar{\beta} \operatorname{com} \bar{\beta}(0)=p$. Sobre a geodésica $\bar{\beta}$, tome os pontos $\bar{\beta}\left(t_{n}^{\prime}\right)$ tais que

$$
d_{F}\left(\bar{\beta}, \beta\left(-t_{n}\right)\right)=d_{F}\left(\bar{\beta}\left(t_{n}^{\prime}\right), \beta\left(-t_{n}\right)\right)
$$

Pelos Lema 2.20, $d_{F}\left(\bar{\beta}\left(t_{n}^{\prime}\right), \beta\left(-t_{n}\right)\right) \leqslant C$ para algum $C$ independente de $n$. Note que $\varangle_{p}\left(\bar{\beta}^{\prime}(0), \sigma^{\prime}(0)\right)>0$, pois caso contrário $\bar{\beta}=\sigma$ e teríamos $h(x)=y$ (Figura 3.2). O axioma de visibilidade para $t>0$ aplicado aos raios geodésicos $\bar{\beta}$ e $\sigma$ nos diz que existe $\bar{q}_{n} \in\left[\bar{\beta}\left(t_{n}^{\prime}\right), \sigma\left(t_{n}\right)\right]$ tal que $d_{F}\left(p, \bar{q}_{n}\right) \leqslant B$ para algum $B>0$.

Afirmamos que:

- Existe $q_{n} \in \gamma_{n}$ tal que $d_{F}\left(\bar{q}_{n}, q_{n}\right) \leqslant \delta$. 


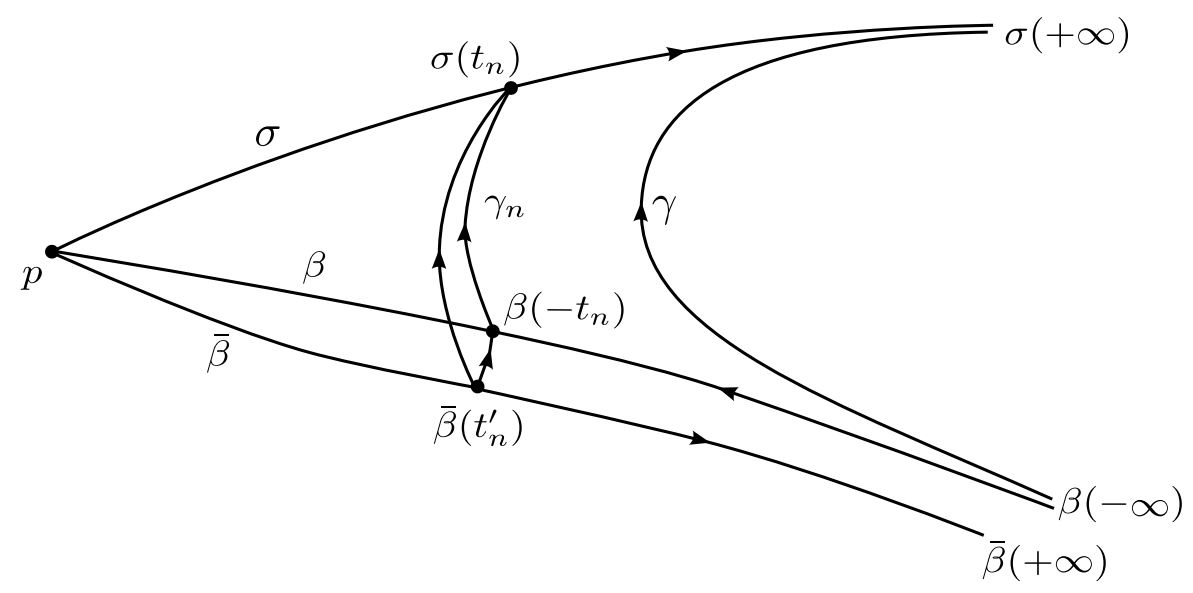

Figura 3.2: Construindo a sequência $\gamma_{n}$.

De fato, como $\tilde{M}$ é Gromov-Finsler $\delta$-hiperbólico, $d_{F}\left(\bar{q}_{n},\left[\bar{\beta}\left(t_{n}^{\prime}\right), \beta\left(-t_{n}\right)\right] \cup\right.$ $\left.\gamma_{n}\right) \leqslant \delta$ para todo $n$. Mas $\bar{\beta}\left(t_{n}^{\prime}\right) \rightarrow \bar{\beta}(+\infty)$ e $L_{\tilde{F}}\left(\left[\bar{\beta}\left(t_{n}^{\prime}\right), \beta\left(-t_{n}\right)\right]\right) \leqslant C$ implicam que $d_{\tilde{F}}\left(\bar{q}_{n},\left[\bar{\beta}\left(t_{n}^{\prime}\right), \beta\left(-t_{n}\right)\right]\right) \rightarrow \infty$. Logo, a partir de um certo $n_{0}$,

$$
d_{\tilde{F}}\left(\bar{q}_{n},\left[\bar{\beta}\left(t_{n}^{\prime}\right), \beta\left(-t_{n}\right)\right] \cup \gamma_{n}\right)=d_{\tilde{F}}\left(\bar{q}_{n}, \gamma_{n}\right) \leqslant \delta,
$$

ou seja, para $n>n_{0}$ existe $q_{n} \in \gamma_{n}$ tal que $d_{\tilde{F}}\left(\bar{q}_{n}, q_{n}\right) \leqslant \delta$.

- A menos de considerar uma subsequência, $\gamma_{n}$ converge uniformemente em compactos para uma geodésica $\gamma$.

Pela desigualdade triangular, para $n>n_{0}$ temos

$$
d_{\tilde{F}}\left(p, q_{n}\right) \leqslant d_{\tilde{F}}\left(p, \bar{q}_{n}\right)+d_{\tilde{F}}\left(\bar{q}_{n}, q_{n}\right) \leqslant B+\delta
$$

ou seja, a sequência $q_{n}$ é limitada e então, considerando uma subsequência se necessário, existe uma geodésica $\gamma$ tal que $\gamma_{n} \rightarrow \gamma$ uniformemente em compactos.

Falta verificarmos que $\gamma(-\infty)=x$ e $\gamma(+\infty)=y$. Sejam $q=\lim _{n \rightarrow \infty} q_{n}$ e $w$ o vetor unitário tangente a $\gamma$ em $q$. Por um lado, $V^{-}\left(\beta\left(-t_{n}\right), q_{n}\right) \rightarrow w$ e $\beta\left(-t_{n}\right) \rightarrow \beta(-\infty)$ implicam que $\beta$ e $\gamma$ são b-assintóticas pelo lema 3.7. Por outro lado, $V^{+}\left(q_{n}, \sigma\left(t_{n}\right)\right) \rightarrow w$ e $\sigma\left(t_{n}\right) \rightarrow \sigma(+\infty)$ implicam que $\sigma$ e $\gamma$ são f-assintóticas, também pelo lema 3.7 .

A construção da geodésica $\bar{\beta}$ no argumento acima é necessária porque nossa definição de visibilidade considera ambos os raios geodésicos partindo de (ou chegando em) um mesmo ponto, o que não era o caso para $\beta$ e $\sigma$ (a visibilidade foi usada com $\bar{\beta}$ e $\sigma$ e não $\operatorname{com} \beta$ e $\sigma$ ). Vale notar que a Proposição 2.20 cumpre um papel fundamental na obtenção da geodésica limite $\gamma$. Para efeito de comparação técnica, no trabalho de Eberlein ([6], Proposition 1.7) a 
construção de $\bar{\beta}$ segue diretamente da reversibilidade das geodésicas e da noção usual de visibilidade em variedades Riemannianas. Além disso, observamos que Eberlein exige como hipótese que os pontos $x, y \in \partial \tilde{M}(\infty)$ cumpram $x \neq y$. Esta condição é naturalmente traduzida para nosso contexto como $h(x) \neq y$.

\section{4}

Geometria global de $\Gamma_{S}\left(\pi_{1}(M)\right)$

Sabemos pelo Teorema 2.22 que se $(M, F)$ é uma variedade Finsler compacta, sem pontos conjugados e de visibilidade uniforme, então $\left(\tilde{M}, d_{\tilde{F}}\right)$ é um espaço Gromov-Finsler $\delta$-hiperbólico. Provaremos a seguir que o grafo de Cayley de $\pi_{1}(M)$ é naturalmente quase-isométrico a $\tilde{M}$. Usando a Proposição 2.12 , concluiremos que $\pi_{1}(M)$ é um grupo finitamente gerado cujo grafo de Cayley é Gromov-Finsler $\delta$-hiperbólico e, portanto $\pi_{1}(M)$ é um grupo Gromov $\delta$-hiperbólico (pois a métrica da palavra é simétrica). Usando os teoremas de Gromov enunciados no final do Capítulo 2, poderemos fazer uma acurada descrição da ação de $\pi_{1}(M)$ em $\partial \tilde{M}(+\infty) \times \partial \tilde{M}(+\infty)$.

\subsection{1}

\section{$\left(\tilde{M}, d_{\tilde{F}}\right)$ é quase-isométrico a $\left(\Gamma_{S}\left(\pi_{1}(M), d\right)\right.$}

Sejam $S=S^{-} \cup S^{+}$um gerador simétrico de $\pi_{1}(M), p_{0} \in S$ um ponto base a partir do qual $\Gamma_{S}\left(\pi_{1}(M)\right)$ é construído e $\tilde{p}_{0} \in \tilde{M}$ um ponto fixado. Analogamente à construção do grafo de Cayley, ligue $\tilde{p}_{0}$ a cada ponto de $A_{1}\left(\tilde{p}_{0}\right):=\left\{a\left(\tilde{p}_{0}\right), a \in S\right\}$ com um segmento de geodésica unitária, escolhendo a sua orientação segundo o critério:

- se $a \in S^{+}$, ligue com $\left[\tilde{p}_{0}, a\left(\tilde{p}_{0}\right)\right]$;

- se $a \in S^{-}$, ligue com $\left[a\left(\tilde{p}_{0}\right), \tilde{p}_{0}\right]$,

Defina recursivamente os conjuntos

$$
A_{j+1}\left(\tilde{p}_{j}\right):=\left\{a \cdot \tilde{p}_{j} / \tilde{p}_{j} \in A_{j} \text { e } a \in S\right\}
$$

e ligue o ponto $\tilde{p}_{j} \in A_{j}$ a cada $a\left(\tilde{p}_{j}\right) \in A_{j+1}$ com uma geodésica definida em $[0,1]$, orientada segundo o critério:

- se $a \in S^{+}$, ligue com $\left[\tilde{p}_{j}, a\left(\tilde{p}_{j}\right)\right]$;

- se $a \in S^{-}$, ligue com $\left[a\left(\tilde{p}_{j}\right), \tilde{p}_{j}\right]$.

Isso produz uma espécie de "cópia orientada" de $\Gamma_{S}\left(\pi_{1}(M)\right)$ contida em $\tilde{M}$, como ilustramos na Figura 3.3. 


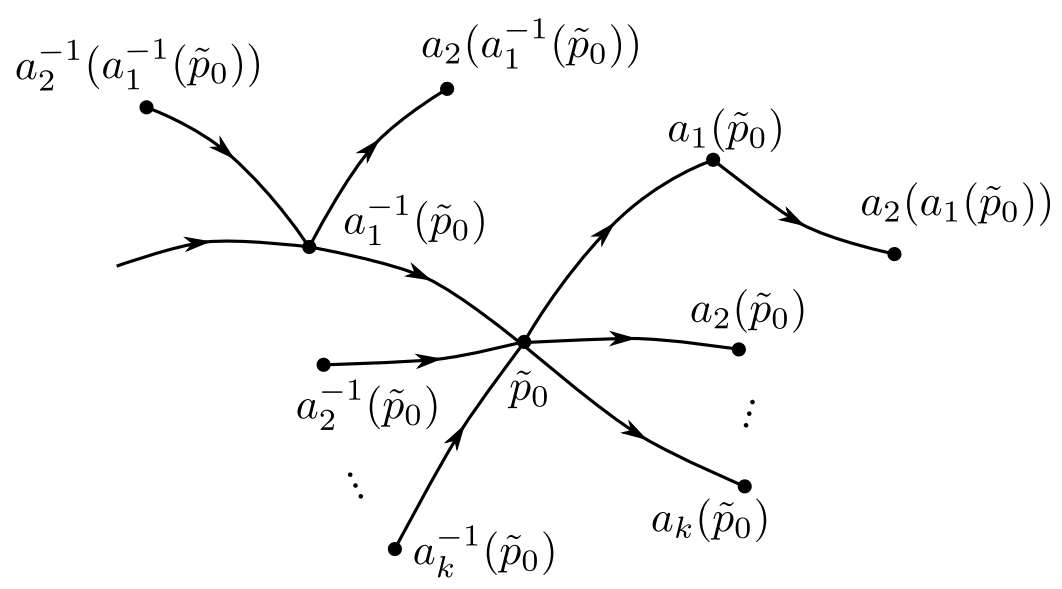

Figura 3.3: Uma cópia "orientada" do grafo de Cayley em $\tilde{M}$.

Agora construiremos uma quase-isometria $f: \Gamma_{S}\left(\pi_{1}(M)\right) \longrightarrow \tilde{M}$ recursivamente. Seja $B_{j+1}$ o conjunto $A_{j+1}$ reunido com as arestas $\left[p_{j}, a\left(p_{j}\right)\right] \mathrm{e}$ $\left[a\left(p_{j}\right), p_{j}\right]$. Temos que

$$
\Gamma_{S}\left(\pi_{1}(M)\right)=\bigcup_{j \in \mathbb{N}} B_{j}
$$

Definiremos a quase-isometria nos pontos de $B_{j}$ para cada $j \in \mathbb{N}$. Naturalmente,

$$
f\left(p_{0}\right):=\tilde{p}_{0}
$$

Seja $\tilde{p} \in B_{1}$. Identificando as arestas e vértices de $B_{1}$ com o intervalo $[0,1]$, temos que $\tilde{p} \in[0,1]$.

- Se $a \in S^{+}$, associe a $\tilde{p}$ o ponto $f(\tilde{p}):=\left[\tilde{p}_{0}, a\left(\tilde{p}_{0}\right)\right](\tilde{p})$;

- Se $a \in S^{-}$, associe a $\tilde{p}$ o ponto $f(\tilde{p}):=\left[a\left(\tilde{p}_{0}\right), \tilde{p}_{0}\right](1-\tilde{p})$.

Em seguida, seja $p \in B_{j+1}$. Então $p \in[0,1]$, que é uma 1-célula ligando $p_{j} \in A_{j}$ ao ponto $a \cdot p_{j} \in A_{j+1}$. Novamente:

- Se $a \in S^{+}$, associe a $p$ o ponto $f(p):=\left[\tilde{p}_{0}, a\left(\tilde{p}_{0}\right)\right](p)$;

- Se $a \in S^{-}$, associe a $p$ o ponto $f(p):=\left[a\left(\tilde{p}_{0}\right), \tilde{p}_{0}\right](1-p)$.

Isso encerra a construção de $f: \Gamma_{S}\left(\pi_{1}(M)\right) \longrightarrow \tilde{M}$.

Proposição 3.13. A função $f: \Gamma_{S}\left(\pi_{1}(M)\right) \longrightarrow \tilde{M}$ acima é uma $(A, 0)$-quaseisometria entre os espaços métricos Finsler $\left(\Gamma_{S}\left(\pi_{1}(M)\right), d\right)$ e $\left(\tilde{M}, d_{\tilde{F}}\right)$.

Demonstração. Segue diretamente da construção acima e da condição $d_{\tilde{F}}(x, y) \leqslant C d_{\tilde{F}}(y, x)$. A quase-inversa de $f$ será a função $\tilde{f}: \tilde{M} \longrightarrow \Gamma_{S}\left(\pi_{1}(M)\right)$ assim definida: seja $U \subset \tilde{M}$ um domínio fundamental do recobrimento tal que $\tilde{p}_{0} \in U$. Então,

$$
\tilde{M}=\bigcup_{a \in \pi_{1}(M)} a(U)
$$


com $a(U) \cap \bar{a}(U) \neq \varnothing$ se e somente se $a=\bar{a}$ (e neste caso $a(U)=\bar{a}(U)$ ). Além disso, $U$ é contrátil a $p_{0}$. Basta definir então $\tilde{f}(\tilde{p})=a \cdot p_{0}$ quando $\tilde{p} \in a(U)$. As funções $f$ e $\tilde{f}$ satisfazem às condições da Definição 2.2 .

\section{4 .2}

\section{Consequências da quase-isometria $f$}

Corolário 3.14. Seja $(M, F)$ uma variedade Finsler compacta, sem pontos conjugados e de visibilidade uniforme. Então $\left(\Gamma_{S}\left(\pi_{1}(M)\right), d\right)$ é espaço métrico Gromov-Finsler $\delta$-hiperbólico, para algum $\delta>0$.

Demonstração. Pelo Teorema 2.22, $(\tilde{M}, \tilde{F})$ é um espaço métrico GromovFinsler $\delta^{\prime}$-hiperbólico para algum $\delta^{\prime}>0$. Pela proposição anterior, $(\tilde{M}, \tilde{F})$ e $\left(\Gamma_{S}\left(\pi_{1}(M)\right), d\right)$ são quase-isométricos. Pela Proposição 2.12, $\left(\Gamma_{S}\left(\pi_{1}(M)\right), d\right)$ é Gromov-Finsler $\delta$-hiperbólico para algum $\delta>0$.

Sob as mesmas hipóteses do corolário anterior, concluímos que $\pi_{1}(M)$ é um grupo hiperbólico no sentido da Definição 2.26.

Proposição 3.15. Seja $(M, F)$ uma variedade Finsler compacta, sem pontos conjugados e de visibilidade uniforme. Então $\pi_{1}(M)$ é um grupo Gromov $\delta$ hiperbólico, para algum $\delta>0$.

Demonstração. Pelo corolário anterior, $\left(\Gamma_{S}\left(\pi_{1}(M)\right), d\right)$ é um espaço métrico Gromov-Finsler $\delta$-hiperbólico. Como $d$ satisfaz $d(x, y)=d(y, x)$, temos que $\left(\Gamma_{S}\left(\pi_{1}(M)\right), d\right)$ é um espaço métrico Gromov $\delta$-hiperbólico e portanto $\pi_{1}(M)$ é um grupo Gromov $\delta$-hiperbólico para algum $\delta>0$.

Com isso, usando os teoremas de Gromov sobre a ação de um grupo Gromov $\delta$-hiperbólico no bordo de seu grafo de Cayley (Teoremas 2.29 a 2.31), obtemos a importante consequência abaixo:

Corolário 3.16. Seja $(M, F)$ uma variedade Finlser compacta, sem pontos conjugados, tal que $(\tilde{M}, \tilde{F})$ é de visibilidade uniforme. Então a ação $\pi_{1}(M)$ em $\partial \Gamma_{S}\left(\pi_{1}(M)\right) \times \partial \Gamma_{S}\left(\pi_{1}(M)\right)$ tem uma órbita densa.

Demonstração. Uma vez que $\pi_{1}(M)$ é Gromov $\delta$-hiperbólico, o resultado segue do Teorema 2.31. 


\section{5}

\section{Dualidade de Eberlein no contexto Finsler}

Definiremos a seguir uma extensão Finsler para o conceito de dualidade introduzido por Eberlein ([7], Definition 8.2). Esta noção estabelecerá adiante a conexão entre as ações de $\pi_{1}(M)$ em $\partial \tilde{M}(-\infty) \times \partial \tilde{M}(+\infty)$ com a transitividade do fluxo geodésico de $(M, F)$ (Proposição 3.30). O homeomorfismo $h$ construído na seção 3.2 desempenhará um importante papel em nossa extensão da definição de dualidade. É interessante notar que a construção $h$ foi possível devido à Proposição 2.20, evidenciando a relevância de se obter um controle global da "largura" dos bígonos geodésicos.

Definição 3.17. Dizemos que $x \in \partial \tilde{M}(-\infty)$ é dual a $y \in \partial \tilde{M}(+\infty)$ se para toda vizinhança aberta $V$ de $x$ e para toda vizinhança aberta $W$ de y existe uma isometria de recobrimento $\phi \in \pi_{1}(M)$ tal que $\phi(\tilde{M}(-\infty)-V) \subset h^{-1}(W)$.

É claro que $\phi(\tilde{M}(-\infty)-V) \subset h^{-1}(W)$ se e só se $\phi(\tilde{M}(+\infty)-W) \subset$ $h(V)$. Portanto, diremos sem maiores comentários que $y \in \partial \tilde{M}(+\infty)$ é dual a $x \in \partial \tilde{M}(-\infty)$ sempre que $x$ for dual a $y$. Dessas observações segue imediatamente o seguinte:

Corolário 3.18. Se $x \in \partial \tilde{M}(-\infty)$ é dual a $y \in \partial \tilde{M}(+\infty)$, então para toda isometria $\phi \in \pi_{1}(M)$ tem-se que $x \in \phi(\tilde{M}(-\infty)-V)$ se e somente se $h(x) \in \phi(\tilde{M}(+\infty)-h(V))$.

Os pares duais têm as seguintes propriedades:

Proposição 3.19. 1. Fixado $x \in \partial \tilde{M}(-\infty)$, o conjunto dos pontos $y \in$ $\partial \tilde{M}(+\infty)$ duais a $x$ é fechado e invariante pela ação de $\pi_{1}(M)$.

2. $x \in \partial \tilde{M}(-\infty)$ e $y \in \partial \tilde{M}(+\infty)$ são duais se e somente se $h(x)$ e $h^{-1}(y)$ são duais.

Demonstração. Para provar 1 , sejam $y_{n} \in \partial \tilde{M}(+\infty)$ pontos duais a $x$ e $V$ um aberto em torno de $x$. Suponha que $y_{n} \rightarrow y$. Se $W$ é um aberto contendo $y$, então $y_{n} \in W$ para $n>n_{0}$ suficientemente grande. Pela dualidade de $x$ e $y_{n}$, existe $\phi \in \pi_{1}(M)$ tal que $\phi(\tilde{M}(-\infty)-V) \subset h^{-1}(W)$. Como $V$ e $W$ são arbitrários, $x$ e $y$ são duais.

Agora seja $\psi \in \pi_{1}(M)$ uma isometria do recobrimento e suponha que $y \in \partial \tilde{M}(+\infty)$ é dual a $x$. Dados abertos arbitrários $V$ e $W$ em torno de $x$ e $\psi(y)$ respectivamente, existe $\phi \in \pi_{1}(M)$ tal que $\phi(\tilde{M}(-\infty)-V) \subset h^{-1}\left(\psi^{-1}(W)\right)$. Então a isometria $\xi=\psi \circ \phi$ satisfaz $\xi(\tilde{M}(-\infty)-V) \subset h^{-1}(W)$ e portanto $x$ e $\psi(y)$ são duais. 
A demonstração de 2 segue diretamente da definição de dualidade e do fato de $h$ ser um homeomorfismo equivariante com respeito às ações de $\pi_{1}(M)$ em $\partial \tilde{M}(-\infty)$ e em $\partial \tilde{M}(+\infty)$.

\subsection{1}

\section{Pontos limites são duais}

Denotaremos por

$$
L^{-}\left(\pi_{1}(M)\right) \quad \text { e } \quad L^{+}\left(\pi_{1}(M)\right)
$$

os conjuntos de pontos de acumulação em $\tilde{M}(-\infty)$ e em $\tilde{M}(+\infty)$ da órbita de um ponto $p \in \tilde{M}$ pela ação de $\pi_{1}(M)$ em $\tilde{M}(-\infty)$ e em $\tilde{M}(+\infty)$, respectivamente. Sob a hipótese de visibilidade, tais conjuntos independem da escolha de $p$. Como essas órbitas não têm pontos de acumulação em $\tilde{M}$, segue que $L^{-}\left(\pi_{1}(M)\right) \subset \partial \tilde{M}(-\infty)$ e $L^{+}\left(\pi_{1}(M)\right) \subset \partial \tilde{M}(+\infty)$ e ambos são não-vazios.

Proposição 3.20. Seja $x \in \partial \tilde{M}(-\infty)$ e $U \subset \tilde{M}(-\infty)$ uma vizinhança de $x$. $S e p_{n} \in \tilde{M}$ é uma sequência convergindo para $x$, então $\varangle_{p_{n}}(\tilde{M}(-\infty)-U) \rightarrow 0$. Demonstração. É suficiente mostrar que para $p \in \tilde{M}(-\infty)-U$ e para uma sequência arbitrária $a_{n} \in \tilde{M}(-\infty)-U$ vale $\varangle_{p_{n}}\left(p, a_{n}\right) \rightarrow 0$. É também suficiente considerarmos $U$ um cone truncado em torno de $x$. Existe $n_{0}$ e $\varepsilon>0$ tais que $n>n_{0}$ implica $\varangle_{p}\left(p_{n}, a_{n}\right) \geqslant \varepsilon$. Se $\sigma_{n}$ é a única geodésica orientada ligando $p_{n}$ a $a_{n}$, a visibilidade uniforme nos diz que existe $R>0$ tal que $d_{F}\left(p, \sigma_{n}\right) \leqslant R$. Sejam $q_{n} \in \sigma_{n}$ tais que $d_{F}\left(p, q_{n}\right) \leqslant R$. Como $d_{F}\left(p_{n}, p\right) \rightarrow \infty$, então novamente pela visibilidade uniforme (aplicada ao ângulo formado por $\left[p_{n}, p\right]$ e $\left.\left[p_{n}, q_{n}\right]\right)$ temos $\varangle_{p_{n}}\left(p, q_{n}\right) \rightarrow 0$. Mas $\varangle_{p_{n}}\left(p, q_{n}\right)=\varangle_{p_{n}}\left(p, a_{n}\right)$ e portanto $\varangle_{p_{n}}\left(p, a_{n}\right) \rightarrow 0$.

A propriedade fundamental dos pares duais é a seguinte:

Proposição 3.21. Seja $(\tilde{M}, \tilde{F})$ o recobrimento universal de uma variedade Finsler compacta, sem pontos conjugados e de visibilidade uniforme. Os pontos $x \in \partial \tilde{M}(-\infty)$ e $y \in \partial \tilde{M}(+\infty)$ são duais se e somente se existe uma sequência de isometrias $\phi_{n} \in \pi_{1}(M)$ tal que para todo ponto $p \in \tilde{M}$ tem-se $\phi_{n}^{-1}(p) \rightarrow x$ $e \phi_{n}(p) \rightarrow y$ quando $n \rightarrow \infty$.

Demonstração. Suponha que $x$ e $y$ são duais e sejam $\left\{U_{n}\right\}$ e $\left\{V_{n}\right\}$ bases de abertos em torno de $x$ e $y$ respectivamente. Para cada $n$, seja $\phi_{n} \in \pi_{1}(M)$ tal que $\phi_{n}\left(\tilde{M}(-\infty)-U_{n}\right) \subset h^{-1}\left(V_{n}\right)$ e $\phi_{n}^{-1}\left(\tilde{M}(+\infty)-V_{n}\right) \subset h\left(U_{n}\right)$. Afirmamos que $\phi_{n}$ é a sequência procurada. De fato, seja $p \in \tilde{M}$ tal que $p \notin U_{n}$ e $p \notin V_{n}$. Então para todo $n$ valem 


$$
\phi_{n}(p) \in h^{-1}\left(V_{n}\right) \quad \text { e } \quad \phi_{n}^{-1}(p) \in h\left(U_{n}\right)
$$

o que implica

$$
\phi_{n}(p) \rightarrow h^{-1}(y) \quad \text { e } \quad \phi_{n}^{-1}(p) \rightarrow h(x)
$$

Pela Proposição 3.19 concluimos que $\phi_{n}(p) \rightarrow y$ e $\phi_{n}^{-1}(p) \rightarrow x$, como queríamos provar.

Reciprocamente, sejam $\phi_{n} \in \pi_{1}(M)$ tais que $\phi_{n}^{-1}(p) \rightarrow x$ e $\phi_{n}(p) \rightarrow y$. Considere vizinhanças $U \subset \tilde{M}(-\infty)$ de $x$ e $V \subset \tilde{M}(+\infty)$ de $y$. Pela Proposição 3.20 e pelo Lema 2.16 , temos que

$$
\varangle_{p} \phi_{n}(\tilde{M}(-\infty)-U)=\varangle_{\phi^{-1}(p)}(\tilde{M}(-\infty)-U) \rightarrow 0
$$

para qualquer ponto $p \in \tilde{M}$. Podemos supor que $p \in \tilde{M}(-\infty)-U$. Como $\phi_{n}^{-1}(p) \rightarrow x$, então $d_{F}\left(\phi_{n}^{-1}(p), \tilde{M}(-\infty)\right) \rightarrow \infty$. Pela Proposição 3.19 e pela hipótese $\phi_{n}(p) \rightarrow y$, temos que $\phi_{n}(p) \rightarrow h^{-1}(y) \in \partial \tilde{M}(-\infty)$. Segue que $\phi_{n}(\tilde{M}(-\infty)-U) \subset h^{-1}(V)$. Como $U$ e $V$ são vizinhanças arbitrárias, temos que $x$ e $y$ são duais.

Corolário 3.22. 1. Todo ponto $x \in L^{-}\left(\pi_{1}(M)\right)$ tem um ponto dual $y \in$ $L^{+}\left(\pi_{1}(M)\right)$.

2. Os conjuntos $L^{-}\left(\pi_{1}(M)\right)$ e $L^{+}\left(\pi_{1}(M)\right)$ são fechados em $\partial \tilde{M}(-\infty)$ e em $\partial \tilde{M}(+\infty)$ respectivamente e invariantes pela ação de $\pi_{1}(M)$.

Demonstração. Para o item 1 , se $x \in L^{-}\left(\pi_{1}(M)\right)$, então existe uma sequência $\phi_{n} \in \pi_{1}(M)$ tal que $\phi_{n}^{-1}(p) \rightarrow x$ para todo $p \in \tilde{M}$. Mas a sequência $\phi_{n}(p)$ tem ponto de acumulação e portanto, a menos de considerarmos uma subsequência, existe $y \in L^{+}\left(\pi_{1}(M)\right)$ tal que $\phi_{n}(p) \rightarrow y$.

Para o item 2, basta usar um argumento padrão de escolha de subsequência diagonal: seja $x_{j} \rightarrow x \operatorname{com} x_{j} \in L^{-}\left(\pi_{1}(M)\right)$. Para cada $j$, existe $\phi_{n j} \in \pi_{1}(M)$ tal que $\lim _{n \rightarrow \infty} \phi_{n j}(p)=x_{j}$. As sequências $\phi_{n j}(p)$ podem ser escolhidas de tal forma que $\phi_{n n}(p) \rightarrow x$. Analogamente para $x_{j} \in L^{+}\left(\pi_{1}(M)\right)$.

A invariância de $L^{-}\left(\pi_{1}(M)\right)$ e $L^{+}\left(\pi_{1}(M)\right)$ segue diretamente da definição da extensão da ação de $\pi_{1}(M)$ em $\partial \tilde{M}(-\infty)$ e $\partial \tilde{M}(+\infty)$

Corolário 3.23. Os pares duais estão em $L^{-}\left(\pi_{1}(M)\right) \times L^{+}\left(\pi_{1}(M)\right)$.

Demonstração. Segue diretamente da Proposição 3.21. 


\section{5 .2}

Pontos de $\partial \tilde{M}(-\infty) \times \partial \tilde{M}(+\infty)$ são duais

Provaremos ao final desta subseção que todos os pares $(a, b) \in \partial \tilde{M}(-\infty) \times$ $\partial \tilde{M}(+\infty)$ são duais. Para demonstrar este fato, desenvolveremos a argumentação a partir de agora para mostrar que $L^{-}\left(\pi_{1}(M)\right) \times L^{+}\left(\pi_{1}(M)\right)=$ $\partial \tilde{M}(-\infty) \times \partial M(+\infty)$.

Lema 3.24. Se $\phi$ tem pontos fixos distintos $x, y \in \partial \tilde{M}(-\infty)$, então (trocando $\phi^{n}$ por $\phi^{-n}$ se necessário) $\phi^{n}(q) \rightarrow x$ e $\phi^{-n}(q) \rightarrow y$ para todo $q \in \tilde{M}$.

Demonstração. Sendo $x \neq y$, pela Proposição 3.12 existe uma geodésica $\gamma$ tal que $\gamma(-\infty)=x$ e $\gamma(+\infty)=h(y)=: \bar{y}$. Podemos escolher um ponto $p \in \tilde{M}$ tal que $\varangle_{p}(x, \bar{y})=\kappa$ para um $\kappa>0$ fixado (ver página 32, logo após a Definição 2.15). Como $x, \bar{y}$ são pontos fixos de $\phi$, pelo Lema 2.16

$$
\varangle_{\phi^{n}(p)}(x, \bar{y})=\varangle_{p}\left(\phi^{-n}(x), \phi^{-n}(\bar{y})\right)=\kappa .
$$

Pela visibilidade uniforme, existe $R>0$ tal que

$$
\max \left\{d_{F}\left(\phi^{n}(p), \gamma\right), d_{F}\left(\gamma, \phi^{n}(p)\right)\right\} \leqslant R
$$

para todo $n \in \mathbb{Z}$. Sejam $s_{n}, t_{n} \in \mathbb{R}$ tais que

$$
d_{F}\left(\gamma\left(s_{n}\right), \phi^{n}(p)\right) \leqslant R \quad \text { e } \quad d_{F}\left(\phi^{-n}(p), \gamma\left(t_{n}\right)\right) \leqslant R .
$$

para $n \in \mathbb{N}$. Como a ação de $\pi_{1}(M)$ é própria e descontínua, as sequências $s_{n}$ e $t_{n}$ não podem ter pontos de acumulação, pois caso contrário a sequência $\phi^{n}(p)$ teria ponto de acumulação em $\tilde{M}$, contrariando a descontinuidade da ação. Vamos supor por exemplo que existe uma subsequência $s_{n_{j}}$ de $s_{n}$ com $s_{n_{j}} \rightarrow \infty$. Então, reindexando $s_{n_{j}}$ e trocando se necessário a ordem de $s_{n}$ com $s_{n+1}$ para que a notação $\left[s_{n}, s_{n+1}\right]$ faça sentido, temos que

$$
[0, \infty) \subset \bigcup_{n \geqslant 0}\left[s_{n}, s_{n+1}\right]=: \mathcal{A}
$$

onde estamos considerando $s_{0}=0$. Ao mesmo tempo, usando a constante $C>1$ definida em (1-3) e a desigualdade triangular,

$$
\begin{aligned}
\left|s_{n}-s_{n+1}\right| & =\min \left\{d_{F}\left(\gamma\left(s_{n}\right), \gamma\left(s_{n+1}\right)\right), d_{F}\left(\gamma\left(s_{n+1}\right), \gamma\left(s_{n}\right)\right\}\right. \\
& =C d_{F}\left(\gamma\left(s_{n}\right), \gamma\left(s_{n+1}\right)\right) \\
& \leqslant d_{F}\left(\gamma\left(s_{n}\right), \phi^{n}(p)\right)+d_{F}\left(\phi^{n}(p), \phi^{n+1}(p)\right)+d_{F}\left(\phi^{n+1}(p), \gamma\left(s_{n+1}\right)\right) \\
& \leqslant R+d_{F}(p, \phi(p))+C R \\
& \leqslant 2 C R+d_{F}(p, \phi(p))=: B .
\end{aligned}
$$


Como a ação de $\pi_{1}(M)$ é descontínua, a sequência $\left\{\phi^{n}(p)\right\}_{n \in \mathbb{N}} \subset \tilde{M}$ escapa de qualquer compacto. Portanto, existe $N$ tal que $n \geqslant N$ implica

$$
d_{F}\left(p, \phi^{n}(p)\right) \geqslant 2 C B+2 R \text {. }
$$

Uma vez que para todo $k \geqslant 0$ tem-se

$$
\begin{aligned}
d_{F}\left(\phi^{-k}(p), \phi^{n}(p)\right) & \leqslant d_{F}\left(\phi^{-k}(p), \gamma\left(t_{k}\right)\right)+d_{F}\left(\gamma\left(t_{k}\right), \gamma\left(s_{n}\right)\right)+d_{F}\left(\gamma\left(s_{n}\right), \phi^{n}(p)\right) \\
& \leqslant 2 R+d_{F}\left(\gamma\left(t_{k}\right), \gamma\left(s_{n}\right)\right)
\end{aligned}
$$

e $C\left|t_{k}-s_{n}\right| \geqslant d_{F}\left(\gamma\left(t_{k}\right), \gamma\left(s_{n}\right)\right)$, então para todo $k \geqslant 0$ e para todo $n \geqslant N$ vale

$$
\begin{aligned}
C\left|t_{k}-s_{n}\right| & \geqslant d_{F}\left(\phi^{-k}(p), \phi^{n}(p)\right)-2 R \\
& =d_{F}\left(p, \phi^{n+k}(p)\right)-2 R \\
& \geqslant 2 C B+2 R-2 R=2 C B
\end{aligned}
$$

e portanto $\left|t_{k}-s_{n}\right| \geqslant 2 B$. Logo, é impossível que $\left\{t_{k}\right\}_{k \geqslant 0} \subset \mathcal{A}$, já que os pontos $t_{k}$ teriam que pertencer a algum intervalo $\left[s_{n}, s_{n+1}\right]$, cujo comprimento é no máximo $B$. Temos portanto que $t_{k}<0$ para todo $k$. Como $t_{k}$ não tem pontos de acumulação, concluímos que $t_{k} \rightarrow-\infty$. Sabendo que $d_{F}\left(\phi^{-k}(p), \gamma\left(t_{k}\right)\right) \leqslant R$ e que $\gamma\left(t_{k}\right) \rightarrow \gamma(-\infty)=x$, segue que $\phi^{-k}(p) \rightarrow x$ quando $k \rightarrow \infty$.

Agora que sabemos que $t_{k} \rightarrow-\infty$, podemos repetir o argumento acima considerando

$$
(-\infty, 0] \subset \bigcup_{k \geqslant 0}\left[t_{k+1}, t_{k}\right]=: \mathcal{B}
$$

para provar que $s_{n} \rightarrow+\infty$ e, analogamente, concluir que

$$
\phi^{n}(p) \rightarrow \gamma(+\infty)=\bar{y} \in \partial \tilde{M}(+\infty)
$$

Da equivariância de $h$, temos que $\phi^{n}(p) \rightarrow y \in \partial \tilde{M}(-\infty)$ quando $n \rightarrow \infty$, como queríamos provar. Mas as convergências $\phi^{-k}(p) \rightarrow x$ e $\phi^{n}(p) \rightarrow y$ independem de $p$, pela visibilidade uniforme, encerrando a demonstração do lema.

Seguem imediatamente as seguintes consequências fundamentais:

Corolário 3.25. Seja $\phi \in \pi_{1}(M), \phi \neq I d$. Então $\phi: \tilde{M}(-\infty) \longrightarrow \tilde{M}(-\infty)$ tem exatamente dois pontos fixos distintos em $\partial \tilde{M}(-\infty)$.

Corolário 3.26. Sejam $x, y \in \partial \tilde{M}(-\infty)$ pontos fixos distintos de $\phi \in \pi_{1}(M)$. Então $x, y \in L^{-}\left(\pi_{1}(M)\right)$. Além disso, $(x, h(y)) \in L^{-}\left(\pi_{1}(M)\right) \times L^{+}\left(\pi_{1}(M)\right)$ é um par dual. 
Usaremos os corolários acima e os resultados do Capítulo 2 para mostrar que $L^{-}\left(\pi_{1}(M)\right) \times L^{+}\left(\pi_{1}(M)\right)=\partial \tilde{M}(-\infty) \times \partial \tilde{M}(+\infty)$.

Proposição 3.27. Seja $(M, F)$ uma variedade Finsler compacta, sem pontos conjugados, tal que $(\tilde{M}, \tilde{F})$ é de visibilidade uniforme. Então

$$
L^{-}\left(\pi_{1}(M)\right) \times L^{+}\left(\pi_{1}(M)\right)=\partial \tilde{M}(-\infty) \times \partial \tilde{M}(+\infty) .
$$

Demonstração. Pela Proposição 3.15, $\pi_{1}(M)$ é um grupo Gromov $\delta$-hiperbólico para algum $\delta>0$. Pelo Teorema 2.29, o conjunto

$$
\operatorname{Lim}\left(\pi_{1}(M)\right):=\left\{\left(\phi^{-\infty}, \phi^{+\infty}\right) / \phi \in \pi_{1}(M)\right\}
$$

é denso em $\partial \Gamma_{S}\left(\pi_{1}(M)\right) \times \partial \Gamma_{S}\left(\pi_{1}(M)\right)$, onde $\phi^{ \pm \infty}=\lim _{n \rightarrow \pm \infty} \phi^{n}(x)$ para algum $x \in \Gamma_{S}\left(\pi_{1}(M)\right)$. Uma vez que cada elemento $\phi \in \pi_{1}(M)$ age como homeomorfismo em $\partial \Gamma_{S}\left(\pi_{1}(M)\right), \phi^{ \pm \infty}$ são pontos fixos de $\phi$. Pelo Teorema 2.13, a quase-isometria $f: \Gamma_{S}\left(\pi_{1}(M)\right) \longrightarrow \tilde{M}$ da Proposição 3.13 induz a função

$$
\partial f: \partial \Gamma_{S}\left(\pi_{1}(M)\right) \longrightarrow \partial \tilde{M}(+\infty)
$$

que é um homeomorfismo, pelo Lema 3.5. Em particular,

$$
\left\{\left(f\left(\phi^{-\infty}\right), f\left(\phi^{+\infty}\right)\right) / \phi \in \pi_{1}(M)\right\}
$$

é denso em $\partial \tilde{M}(+\infty) \times \partial \tilde{M}(+\infty)$. Pela Proposição 3.10,

$$
K:=\left\{\left(f\left(\phi^{-\infty}\right),(h \circ f)\left(\phi^{+\infty}\right)\right) / \phi \in \pi_{1}(M)\right\}
$$

é denso em $\partial \tilde{M}(-\infty) \times \partial \tilde{M}(+\infty)$. Pela definição de $f, x:=f\left(\phi^{-\infty}\right)$ e $y:=f\left(\phi^{+\infty}\right)$ são pontos fixos distintos de $\phi$ em $\partial \tilde{M}(-\infty)$. Portanto, pelo Corolário 3.26, temos que $(x, h(y)) \in L^{-}\left(\pi_{1}(M)\right) \times L^{+}\left(\pi_{1}(M)\right)$. Provamos então que

$$
K \subset L^{-}\left(\pi_{1}(M)\right) \times L^{+}\left(\pi_{1}(M)\right) \subset \partial \tilde{M}(-\infty) \times \partial \tilde{M}(+\infty)
$$

e portanto $L^{-}\left(\pi_{1}(M)\right) \times L^{+}\left(\pi_{1}(M)\right)$ é denso em $\partial \tilde{M}(-\infty) \times \partial \tilde{M}(+\infty)$. Como $L^{-}\left(\pi_{1}(M)\right) \times L^{+}\left(\pi_{1}(M)\right)$ é fechado em $\partial \tilde{M}(-\infty) \times \partial \tilde{M}(+\infty)$ (Corolário 3.22), concluímos que $L^{-}\left(\pi_{1}(M)\right) \times L^{+}\left(\pi_{1}(M)\right)=\partial \tilde{M}(-\infty) \times \partial \tilde{M}(+\infty)$.

Encerraremos a seção provando enfim que todos os pares de $\partial \tilde{M}(-\infty) \times$ $\partial \tilde{M}(+\infty)$ são duais.

Proposição 3.28. Todo $\operatorname{par}(x, y) \in \partial \tilde{M}(-\infty) \times \partial \tilde{M}(+\infty)$ é dual. 
Demonstração. Sejam $(x, y) \in \partial \tilde{M}(-\infty) \times \partial \tilde{M}(+\infty)$ e $\bar{z} \in \partial \tilde{M}(+\infty)$ dado pelo Teorema 2.31, cuja órbita $\pi_{1}(\bar{z})$ é densa em $\partial \tilde{M}(+\infty)$. Pelo Corolário 3.22 , existe $z \in \partial \tilde{M}(+\infty)$ dual a $x$. Afirmamos que $x$ é dual a $\bar{z}$. De fato, sejam $U, V$ vizinhanças de $x$ e $\bar{z}$ respectivamente. Como a órbita de $\bar{z}$ é densa em $\partial \tilde{M}(-\infty)$, existe $\phi \in \pi_{1}(M)$ tal que $z^{\prime} \in \phi(V) \cap \partial \tilde{M}(+\infty)$. Pela dualidade, existe $\psi \in \pi_{1}(M)$ tal que $\psi(\tilde{M}(-\infty)-U) \subset h^{-1}(\phi(V))$. Portanto, $\left(\phi^{-1} \circ \psi\right)(\tilde{M}(-\infty)-U) \subset h^{-1}(V)$ e segue que $x$ e $\bar{z}$ são duais.

Seja $D(x)$ o conjunto dos pontos de $\partial \tilde{M}(+\infty)$ duais a $x$. Como $\bar{z} \in D(x)$, pela Proposição 3.19 temos

$$
\pi_{1}(\bar{z}) \subset D(x) \subset \partial \tilde{M}(+\infty)
$$

e consequentemente $D(x)=\partial \tilde{M}(+\infty)$, pois $D(x)$ é fechado. Como $x$ foi tomado arbitrariamente, temos que todo par $(x, y) \in \partial \tilde{M}(-\infty) \times \partial \tilde{M}(+\infty)$ é dual.

\section{6}

\section{Transitividade do fluxo geodésico}

Denote por $\varphi_{t}: \mathcal{S} M \longrightarrow \mathcal{S} M$ o fluxo geodésico da variedade compacta Finsler $(M, F)$. Fixe $\theta \in \mathcal{S} M$ e seja $P^{+}(\theta)$ o conjunto de elementos $\eta \in \mathcal{S} M$ com a seguinte propriedade:

Se $U$ e $V$ são abertos de $\mathcal{S} M$ contendo $\theta$ e $\eta$ respectivamente, então existe uma sequência $t_{n} \rightarrow \infty$ tal que $\varphi_{t_{n}}(U) \cap V \neq \varnothing$ para todo $n$.

Segue diretamente dessa definição que $\eta \in P^{+}(\theta)$ se e somente se existem $\theta_{n} \in \mathcal{S} M$ e $t_{n} \rightarrow \infty$ tais que $\varphi_{t_{n}}\left(\theta_{n}\right) \rightarrow \eta$ quando $n \rightarrow \infty$.

Definição 3.29. $\theta \in \mathcal{S} M$ é dito não-errante quando $\theta \in P^{+}(\theta)$. Denotaremos por $\Omega$ o conjunto de pontos não-errantes de $\mathcal{S} M$.

A relação fundamental entre a dualidade e a transitividade do fluxo geodésico está contida na proposição a seguir. Ela foi enunciada no caso Riemanniano em [6], Lemma 3.5, e a prova para o caso Finsler é uma adaptação natural das ideias lá apresentadas.

Proposição 3.30. Sejam $(\tilde{M}, \tilde{F})$ o recobrimento universal de uma variedade Finsler compacta, sem pontos conjugados e de visibilidade uniforme, $\theta=$ $(p, v), \eta=(q, w) \in \mathcal{S} M$ e $\tilde{\theta}=(\tilde{p}, \tilde{v}), \tilde{\eta}=(\tilde{q}, \tilde{w}) \in \mathcal{S} \tilde{M}$ levantamentos de $\theta, \eta$ pela projeção natural $\pi: \mathcal{S} \tilde{M} \longrightarrow \mathcal{S} M$. Então $\eta \in P^{+}(\theta)$ se e somente se $\gamma_{\tilde{\eta}}(-\infty)$ e $\gamma_{\tilde{\theta}}(+\infty)$ são duais. 
Demonstração. Sejam $\tilde{\varphi}_{t}$ o fluxo geodésico de $(\tilde{M}, \tilde{F})$ restrito a $\mathcal{S} \tilde{M}$ e $\varphi_{t}$ : $\mathcal{S} M \longrightarrow \mathcal{S} M$ o fluxo geodésico projetado. Suponha que $\eta \in P^{+}(\theta)$ e seja $\theta_{n} \rightarrow \theta$ tal que $\varphi_{t_{n}}\left(\theta_{n}\right) \rightarrow \eta$ para alguma sequência $t_{n} \rightarrow \infty$, ou seja,

$$
\gamma_{\theta_{n}}\left(t_{n}\right) \rightarrow q \quad \text { e } \quad \gamma_{\theta_{n}}^{\prime}\left(t_{n}\right) \rightarrow w
$$

Escolha uma sequência $\tilde{\theta}_{n}=\left(\tilde{p}_{n}, \tilde{v}_{n}\right)$ de levantamentos de $\theta_{n}$ tal que $\tilde{\theta}_{n} \rightarrow \tilde{\theta}$. Se $\gamma_{\tilde{\theta}_{n}}$ são as geodésicas do recobrimento tais que $\gamma_{\tilde{\theta}_{n}}(0)=\tilde{p}_{n}$ e $\gamma_{\tilde{\theta}_{n}}^{\prime}(0)=\tilde{v}_{n}$, então para alguma sequência $\phi_{n} \in \pi_{1}(M)$ tem-se que

$$
\left(\phi_{n} \circ \gamma_{\tilde{\theta}_{n}}\right)\left(t_{n}\right) \rightarrow \tilde{q} \quad \text { e } \quad\left(\phi_{n} \circ \gamma_{\tilde{\theta}_{n}}\right)^{\prime}\left(t_{n}\right) \rightarrow \tilde{w}
$$

Como $\tilde{\theta}_{n} \rightarrow \tilde{\theta}$, então $\gamma_{\tilde{\theta}_{n}}\left(t_{n}\right) \rightarrow \gamma_{\tilde{\theta}}(+\infty)$, pela divergência para $t>0$ dos raios geodésicos. Além disso, $d_{F}\left(\gamma_{\tilde{\theta}_{n}}\left(t_{n}\right), \phi_{n}^{-1}(\tilde{q})\right)=d_{F}\left(\left(\phi_{n} \circ \gamma_{\tilde{\theta}_{n}}\right)\left(t_{n}\right), \tilde{q}\right) \rightarrow 0$. Então $\phi_{n}^{-1}(\tilde{q}) \rightarrow \gamma_{\tilde{\theta}}(+\infty)$ na topologia de f-cones.

Vejamos agora que a mesma sequência $\phi_{n}$ satisfaz $\phi_{n}(\tilde{q}) \rightarrow \gamma_{\tilde{\eta}}(-\infty)$. Considere as geodésicas $\alpha_{n}(t)=\left(\phi_{n} \circ \gamma_{\tilde{\theta}_{n}}\right)\left(t+t_{n}\right)$. Por definição,

$$
\alpha_{n}(0)=\left(\phi_{n} \circ \gamma_{\tilde{\theta}_{n}}\right)\left(t_{n}\right) \rightarrow \tilde{q} \quad \text { e } \quad \alpha_{n}^{\prime}(0)=\left(\phi_{n} \circ \gamma_{\tilde{\theta}_{n}}\right)^{\prime}\left(t_{n}\right) \rightarrow \tilde{w}
$$

e portanto $\alpha_{n}\left(-t_{n}\right) \rightarrow \gamma_{\tilde{\eta}}(-\infty)$ na topologia de b-cones. Ao mesmo tempo $\alpha_{n}\left(-t_{n}\right)=\left(\phi_{n} \circ \gamma_{\tilde{\theta}_{n}}\right)(0)=\phi_{n}\left(\tilde{p_{n}}\right)$ e portanto

$$
\phi_{n}^{-1}\left(\alpha_{n}\left(-t_{n}\right)\right)=\tilde{p}_{n} \rightarrow \tilde{p}
$$

ou seja,

$$
d_{F}\left(\alpha_{n}\left(-t_{n}\right), \phi_{n}(\tilde{p})\right)=d_{F}\left(\phi_{n}^{-1}\left(\alpha_{n}\left(-t_{n}\right)\right), \tilde{p}\right) \rightarrow 0 .
$$

Então, $\phi_{n}(\tilde{p}) \rightarrow \gamma_{\tilde{\eta}}(-\infty)$. Como essa convergência independe de $\tilde{p}$ pela visibilidade uniforme, temos que $\phi_{n}(\tilde{q}) \rightarrow \gamma_{\tilde{\eta}}(-\infty)$, como queríamos mostrar.

Reciprocamente, suponhamos que $\gamma_{\tilde{\eta}}(-\infty)$ e $\gamma_{\tilde{\theta}}(+\infty)$ são duais e sejam $\phi_{n} \in \pi_{1}(M)$ tais que para todo $x \in \tilde{M}$ tenhamos $\phi_{n}(x) \rightarrow \gamma_{\tilde{\eta}}(-\infty)$ e $\phi_{n}^{-1}(x) \rightarrow \gamma_{\tilde{\theta}}(+\infty)$. Como tais convergências independem de $x$ (pela visibilidade uniforme), vamos supor que

$$
\phi_{n}(\tilde{p}) \rightarrow \gamma_{\tilde{\eta}}(-\infty) \quad \text { e } \quad \phi_{n}^{-1}(\tilde{q}) \rightarrow \gamma_{\tilde{\theta}}(+\infty) .
$$

Construiremos uma sequência $\theta_{n} \in \mathcal{S} M$ tal que $\theta_{n} \rightarrow \theta$ e $\varphi_{t_{n}}\left(\theta_{n}\right) \rightarrow \eta$ para alguma sequência $t_{n} \rightarrow \infty$. Sejam $t_{n}=d_{F}\left(\phi_{n}(\tilde{p}), \tilde{q}\right)=d_{F}\left(\tilde{p}, \phi_{n}^{-1}(\tilde{q})\right)$ e $\tilde{v}_{n}=V^{+}\left(\phi_{n}(\tilde{p}), \tilde{q}\right)$ (Figura 3.4).

Então $t_{n} \rightarrow \infty$. Pela divergência para $t<0$ de raios geodésicos,

$$
\varphi_{t_{n}}\left(\phi_{n}(\tilde{p}), \tilde{v}_{n}\right)=\left(\tilde{q}, V^{-}\left(\phi_{n}(\tilde{p}), \tilde{q}\right)\right) \rightarrow(\tilde{q}, \tilde{w})
$$




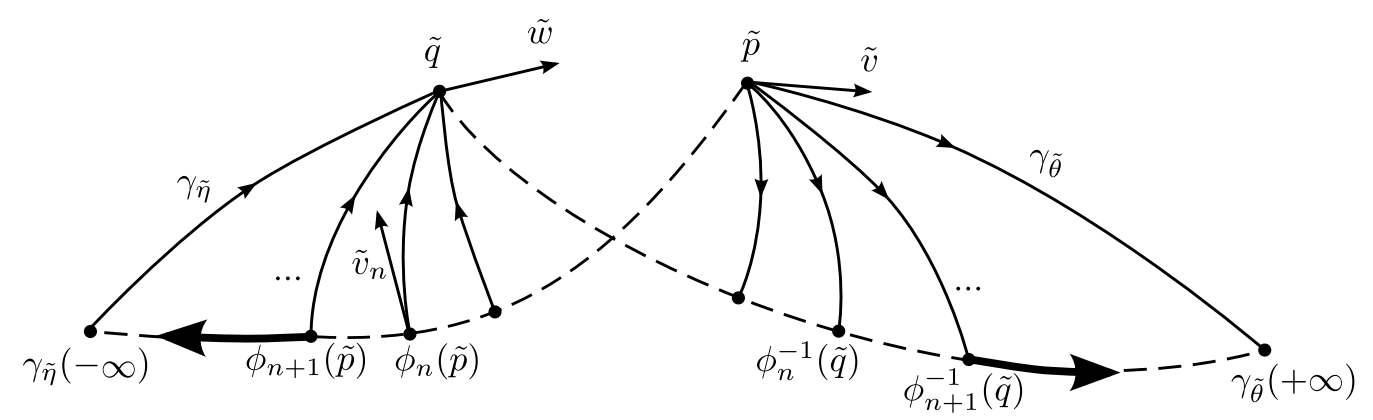

Figura 3.4: Construindo a sequência $\tilde{v}_{n}$.

Como $\phi_{n}^{-1}(\tilde{q}) \rightarrow \gamma_{\tilde{\theta}}(+\infty)$, então $V^{+}\left(\tilde{p}, \phi_{n}^{-1}(\tilde{q})\right) \rightarrow \tilde{v}$ pela divergência para $t>0$ de raios geodésicos. Afirmamos que a sequência $\theta_{n}$ procurada é

$$
\theta_{n}=\pi\left(\phi_{n}(\tilde{p}), \tilde{v}_{n}\right)
$$

De fato, temos que $\theta_{n} \rightarrow \theta$, pois $\pi\left(\phi_{n}(\tilde{p})\right)=\pi(\tilde{p})=p$ para todo $n$. Ao mesmo tempo a geodésica unitária $\left[\phi_{n}(\tilde{p}), \tilde{q}\right](t)$ tem a mesma projeção em $M$ que $\left[\tilde{p}, \phi^{-1}(\tilde{q})\right](t)$, o que implica

$$
(d \pi)_{\phi_{n}(\tilde{p})}\left(V^{+}\left(\phi_{n}(\tilde{p}), \tilde{q}\right)\right)=(d \pi)_{\tilde{p}}\left(V^{+}\left(\tilde{p}, \phi_{n}^{-1}(\tilde{q})\right)\right)
$$

De $V^{+}\left(\tilde{p}, \phi_{n}^{-1}(\tilde{q})\right) \rightarrow \tilde{v}$, segue que $(d \pi)_{\phi_{n}(\tilde{p})}\left(V^{+}\left(\phi_{n}(\tilde{p}), \tilde{q}\right)\right) \rightarrow v$. Concluimos que $\theta_{n} \rightarrow \theta$. Agora vejamos que $\varphi_{t_{n}}\left(\theta_{n}\right) \rightarrow \eta$ :

$$
\begin{aligned}
\varphi_{t_{n}}\left(\theta_{n}\right) & =\left(\varphi_{t_{n}} \circ \pi\right)\left(\phi_{n}(\tilde{p}), V^{+}\left(\phi_{n}(\tilde{p}), \tilde{q}\right)\right) \\
& \left.=\left(\pi \circ \tilde{\varphi}_{t_{n}}\right)\left(\phi_{n}(\tilde{p}), V^{+}\left(\phi_{n}(\tilde{p}), \tilde{q}\right)\right)\right) \\
& \left.=\pi\left(\tilde{q}, V^{-}\left(\phi_{n}(\tilde{p}), \tilde{q}\right)\right)\right) \longrightarrow(q, w)=\eta,
\end{aligned}
$$

pela continuidade de $\pi: T M \longrightarrow M$, concluindo a prova da proposição.

Finalmente provaremos que o fluxo geodésico de uma variedade Finsler compacta, sem pontos conjugados e de visibilidade uniforme é transitivo. Usaremos a proposição abaixo, enunciada por Eberlein [6] no contexto dos fluxos completos em espaços métricos Hausdorff. Reproduziremos o argumento da prova com as notações adotadas em nosso trabalho visando tornar a leitura do capítulo auto-contida.

Proposição 3.31. Suponha que para todo $\theta \in \mathcal{S} M$ tenhamos $P^{+}(\theta)=\mathcal{S} M$. Então o fluxo geodésico $\varphi_{t}$ restrito a $\mathcal{S} M$ é topologicamente transitivo.

Demonstração. Mostraremos que existe $\eta \in \mathcal{S} M$ tal que $\left\{\varphi_{t}(\eta) / t \geqslant 0\right\}$ é denso em $\mathcal{S} M$. Por hipótese, dados $U, V \subset \mathcal{S} M$ abertos, existe uma sequência $t_{n} \rightarrow \infty$ tal que $\varphi_{t_{n}}(U) \cap V \neq \varnothing$. Em particular, fixe um aberto $U$ e escolha 
uma base de abertos $\left\{U_{i}\right\}_{i \in \mathbb{N}}$ para a topologia de $\mathcal{S} M$. Existe $t_{1} \geqslant 1$ tal que $\varphi_{t_{1}}(U) \cap U_{1} \neq \varnothing$. Como $\varphi_{t_{1}}$ é um difeomorfismo, existe um aberto $A_{1} \subset U$ tal que $\bar{A}_{1}$ é compacto e $\varphi_{t_{1}}\left(\bar{A}_{1}\right) \subset U_{1}$. Por hipótese, para o aberto $A_{1}$ existe $t_{2} \geqslant 2$ tal que $\varphi_{t_{2}}\left(A_{1}\right) \cap U_{2} \neq \varnothing$. Escolha $A_{2} \subset U$ aberto tal que $\bar{A}_{2}$ é compacto, $\bar{A}_{2} \subset A_{1}$ e $\phi_{t_{2}}\left(\bar{A}_{2}\right) \subset U_{2}$. Prosseguindo com essa construção, temos que $\bar{A}_{n} \subset A_{n-1}$ e $t_{n} \rightarrow \infty \operatorname{com} \varphi_{t_{n}}\left(\bar{A}_{n}\right) \subset U_{n}$. Como $\bar{A}_{n}$ é uma sequência encaixada de compactos não-vazios, existe $\eta \in \mathcal{S} M$ tal que

$$
\eta \in \bigcap_{n \in \mathbb{N}} \bar{A}_{n}
$$

Afirmamos que $\eta$ satisfaz ao enunciado da proposição. De fato, isso segue diretamente de sua definição, pois $\eta \in \bar{A}_{n}$ para todo $n$ implica

$$
\varphi_{t_{n}}(\eta) \in \varphi_{t_{n}}\left(\bar{A}_{n}\right) \subset U_{n}
$$

Em outras palavras, $\left\{\varphi_{t}(\eta) / t \geqslant 0\right\}$ intersecta todos os abertos de uma base enumerável da topologia de $M$, ou seja, $\left\{\varphi_{t}(\eta) / t \geqslant 0\right\}$ é denso em $\mathcal{S} M$, como queríamos provar.

Teorema A. Seja $(M, F)$ uma variedade Finsler compacta $C^{\infty}$, sem pontos conjugados e de visibilidade uniforme. Então seu fluxo geodésico $\varphi_{t}: \mathcal{S} M \longrightarrow$ $\mathcal{S} M$ é transitivo.

Demonstração. Pela Proposição 3.28, todos os pares de $\partial \tilde{M}(-\infty) \times \partial \tilde{M}(+\infty)$ são duais. Pela Proposição 3.30, $P^{+}(\theta)=\mathcal{S} M$ para todo $\theta \in \mathcal{S} M$ e, finalmente, pela proposição anterior, $\varphi_{t}: \mathcal{S} M \longrightarrow \mathcal{S} M$ é transitivo. 


\section{Aplicações}

O objetivo deste capítulo é provar o resultado abaixo sobre a geometria global de superfícies Finsler compactas, que constitui nossa segunda principal contribuição ao estudo das variedades Finsler:

Teorema A. Seja $(M, F)$ uma superfície Finsler $k$-básica $C^{\infty}$ compacta, sem pontos conjugados, de gênero maior que 1 e cujos fibrados de Green são contínuos. Então $(M, F)$ é Riemanniana.

O resultado acima foi recentemente publicado em [27]. A demonstração deste teorema será dividida em diversos passos. Devemos observar que a hipótese da continuidade dos fibrados de Green é algo forte: de fato, mesmo para variedades Riemannianas tais fibrados podem não ser contínuos - um exemplo disso é dado em em [28].

\section{1}

\section{Campos de Jacobi assintóticos e divergência de raios geodésicos}

Relembramos uma propriedade especial dos campos de Jacobi em variedades sem pontos conjugados: a existência de campos de Jacobi assintóticos, obtidos como limites de campos de Jacobi com um zero. A existência de tais campos para variedades Riemannianas sem pontos conjugados de qualquer dimensão é um resultado conhecido desde o trabalho [5] de Green (veja também [29], Proposition 2.9), assim como para níveis supercríticos de energia de Hamiltonianos convexos sem pontos conjugados (ver por exemplo [30], Proposition $1.10)$.

Recordaremos a construção de campos de Jacobi assintóticos para superfícies Finsler, para a qual precisaremos da divergência futura e passada de campos de Jacobi que zeram em algum ponto. Começaremos com o seguinte resultado de [19] que é provado por Green [5] no caso Riemanniano. Ao longo deste capítulo, $\|\cdot\|$ denotará a norma associada à métrica adaptada $\left(g_{i j}\right)$ nos espaços tangentes de $M$.

Proposição 4.1. Seja $(M, F)$ uma superfície Finsler sem pontos conjugados. Então as soluções não triviais $J(t)$ da equação de Jacobi são uniformemente 
divergentes no seguinte sentido: dado $\varepsilon>0$ e $R>0$, existe $r=r(\varepsilon, R)$ tal que para toda geodésica unitária $\sigma$ e para toda solução $J(t)$ da equação de Jacobi ao longo de $\sigma$ com $J(0)=0$ e $\left\|J^{\prime}(0)\right\| \geqslant \varepsilon$, temos

$$
\|J(t)\| \geqslant R \text { para todo } t \geqslant r .
$$

De fato, com a proposição anterior podemos estabelecer a divergência "passada" para campos de Jacobi que zeram em algum ponto, tal como ocorre no contexto Riemanniano devido à reversibilidade.

Corolário 4.2. Seja $(M, F)$ uma superfície Finsler sem pontos conjugados. Então as soluções não triviais $J(t)$ da equação de Jacobi com $J(0)=0$ são uniformemente divergentes para $t>0$ e para $t<0$ no sentido da Proposição 4.1: dado $\varepsilon>0$ e $R>0$, existe $r=r(\varepsilon, R)$ tal que para toda geodésica unitária $\sigma$ e para toda solução $J(t)$ da equação de Jacobi ao longo de sigma com $J(0)=0$ e $\left\|J^{\prime}(0)\right\| \geqslant \varepsilon$, temos

$$
\|J(t)\| \geqslant R \text { for all }|t| \geqslant R .
$$

Demonstração. Segue da Proposição 4.1 combinada com o fato de que os campos de vetores $W(t)=J(-t)$ são campos de Jacobi da métrica Finsler $G(p, v)=F(p,-v)$, cujas geodésicas são $\alpha(t)=\sigma(-t)$, onde $\sigma$ é geodésica de $(M, F)$.

Agora. seja $\theta=(p, v) \in \mathcal{S} M$ e $\gamma_{\theta}: \mathbb{R} \longrightarrow M$ uma geodésica sem pontos conjugados com $\gamma_{\theta}(0)=p$ e $\gamma_{\theta}^{\prime}(0)=v$, sobre a qual a norma do operador de curvatura é limitada inferiormente por uma constante $K_{0}$. Seja $V \in T_{\gamma_{\theta}(0)} M$ linearmente independente com $\gamma_{\theta}^{\prime}(0)$ e $J_{r}$ uma família de campos de Jacobi cujas codições iniciais são

$$
J_{r}(0)=V \quad \text { e } \quad J_{r}(r)=0 .
$$

Uma vez que a geodésica não tem pontos conjugados, para cada $r \in \mathbb{R}$ existe um único campo de Jacobi $J_{r}$ satisfazendo a essas duas condições. Alem disso, vale o seguinte lema, devido a E.Hopf no caso Riemanniano. Para uma demonstração no contexto Finsler, ver [1], Proposition 2.3.

Lema 4.3. Dada uma geodésica $\gamma: \mathbb{R} \longrightarrow M$ sem pontos conjugados em uma variedade Finsler $(M, F)$, os limites

$$
J_{(\theta, V)}^{+}(t):=\lim _{r \rightarrow+\infty} J_{r}(t) \quad e \quad J_{(\theta, V)}^{-}(t):=\lim _{r \rightarrow-\infty} J_{r}(t)
$$

existem e são campos de Jacobi que não se anulam se $V \neq 0$. 
Os campos de Jacobi definidos pelo lema anterior são chamados, respectivamente de campo de Green-Jacobi estável e campo de Green-Jacobi instável.

Provaremos a partir de agora que superfícies Finsler compactas, sem pontos conjugados e de gênero maior que um são de visibilidade uniforme, o que enquadra tais superfícies nas hipóteses do Teorema A. Começamos com o estudo da divergência de raios geodésicos em nosso contexto.

Como consequência, pelos resultados do capítulo anterior, o fluxo geodésico de uma tal superfície é transitivo. Para mostrar a visibilidade, estudaremos primeiro a divergência de geodésicas que se encontram em um ponto. O resultado a seguir foi provado em [19], Proposition 3.5, e usa a divergência de campos de Jacobi que se anulam em um ponto (aqui demonstrada na Proposição 4.1).

Proposição 4.4 (Divergência de raios geodésicos). Sejam $(M, F)$ uma superfície Finsler de classe $C^{4}$ compacta e sem pontos conjugados e $(\tilde{M}, \tilde{F})$ o seu recobrimento universal com o pull-back da estrutura Finsler pela projeção natural. Então raios geodésicos divergem uniformemente no futuro, ou seja, dados $\varepsilon>0$ e $R>0$, existe $r>0$ tal que para cada par de geodésicas $\beta, \gamma:[0,+\infty) \longrightarrow \tilde{M}$ parametrizadas por comprimento de arco com $\beta(0)=\gamma(0)=p$ e $\varangle_{p}\left(\beta^{\prime}(0), \gamma^{\prime}(0)\right) \geqslant \varepsilon$, tem se que

$$
\inf \left\{d_{\tilde{F}}(\beta(t), \gamma(t)), d_{\tilde{F}}(\gamma(t), \beta(t))\right\} \geqslant R
$$

para todo $t \geqslant r$.

Como consequência, temos também a divergência "passada" de raios geodésicos, enuniciada em [27], e cuja prova fornecemos aqui:

Corolário 4.5. Sejam $(M, F)$ uma superfície Finsler de classe $C^{4}$ compacta e sem pontos conjugados e $(\tilde{M}, \tilde{F})$ o seu recobrimento universal com o pull-back da estrutura Finsler pela projeção natural. Então raios geodésicos divergem uniformemente, ou seja, dados $\varepsilon>0$ e $R>0$, existe $r>0$ tal que para cada par de geodésicas $\beta, \gamma:(-\infty,+\infty) \longrightarrow \tilde{M}$ parametrizadas por comprimento de arco $\operatorname{com} \beta(0)=\gamma(0)=p$ e $\varangle_{p}\left(\beta^{\prime}(0), \gamma^{\prime}(0)\right) \geqslant \varepsilon$, tem se que

$$
\inf \left\{d_{\tilde{F}}(\beta(t), \gamma(t)), d_{\tilde{F}}(\gamma(t), \beta(t))\right\} \geqslant R
$$

para todo $|t| \geqslant r$.

Demonstração. A demonstração segue da proposição anterior combinada com o a observação de que se $J$ é um campo de Jacobi de $(M, F)$, então $W(t)=J(-t)$ é campo de Jacobi de $M$ com a estrutura Finsler $G(p, v)=F(p,-v)$, cujas geodésicas são $\alpha(t)=\gamma(-t)$, onde $\gamma$ é geodésica de $(M, F)$. Portanto, se $(M, F)$ 
não tem pontos conjugados, $(M, G)$ também não tem e seus raios geodésicos divergem para $t \geqslant r$, ou seja, os raios geodésicos de $(M, F)$ divergem para $t \leqslant-r$.

Teorema 4.6. Seja $(M, F)$ uma superfície Finsler compacta, sem pontos conjugados e de gênero maior que um. Então $(\tilde{M}, \tilde{F})$ é de visibilidade uniforme.

Demonstração. Como $M$ é compacta e de gênero maior que um, seu grupo fundamental é Gromov $\delta$-hiperbólico (pois uma tal superfície pode ser munida de uma métric Riemanniana de curvatura constante -1). Como o grupo fundamental de $M$ é quase-isométrico a $\tilde{M}$, segue que $(\tilde{M}, \tilde{F})$ é Gromov-Finsler $\delta$-hiperbólico para algum $\delta>0$, pela Proposição 2.12. Como os raios geodésicos divergem no futuro e no passado pela Proposição 4.5, o Teorema 2.22 mostra que $(\tilde{M}, \tilde{F})$ é de visibilidade uniforme, como queríamos provar.

Consequentemente,

Corolário 4.7. Seja $(M, F)$ uma superfície Finsler compacta, sem pontos conjugados e de gênero maior que um. Então o fluxo geodésico de $(M, F)$ é transitivo.

Demonstração. Segue diretamente do Teorema 4.6 e do Teorema A.

\section{2}

\section{O escalar de Cartan e os campos de Jacobi centrais}

A identidade de Bianchi (ver por exemplo [20]) para métricas Finsler k-básicas nos diz que tais métricas têm a elas associados campos de Jacobi naturalmente definidos. Mais precisamente, seja $f: \mathbb{R} \longrightarrow \mathbb{R}$ uma função que satisfaz à equação escalar de Jacobi ao longo de uma geodésica $\gamma$

$$
f^{\prime \prime}(t)+K\left(\gamma(t), \gamma^{\prime}(t)\right) f(t)=0
$$

Consideremos um campo de vetores e unitário ao longo de $\gamma$, paralelo com respeito à conexão de Chern, e ortogonal a $\gamma$ com respeito à métrica Riemanniana adaptada de $T M-\{0\}$. Defina

$$
J(t):=f(t) e(t) .
$$

Uma vez que $e$ é paralelo (ou seja, $e^{\prime \prime}(t)=0$ para todo $t \in \mathbb{R}$ com respeito à conexão de Chern), é fácil verificar que $J$ assim definido é um campo de Jacobi, o que nos fornece o seguinte resultado, uma consequência da identidade de Bianchi: 
Lema 4.8. Sejam $(M, F)$ uma superfície Finsler $k$-básica, $\gamma_{\theta}: \mathbb{R} \longrightarrow M$ uma geodésica unitária e I o escalar de Cartan de M. Então

1. A função

$$
i(t)=I\left(\gamma_{\theta}(t), \gamma_{\theta}^{\prime}(t)\right)
$$

satisfaz a equação escalar de Jacobi.

2. O campo de vetores

$$
J_{\theta}(t):=i(t) e(t)
$$

é de Jacobi para todo campo de vetores e $(t)$ definido ao longo de $\gamma_{\theta}$ unitário, perpendicular a $\gamma_{\theta}$ com respeito à métrica Riemanniana adaptada de $T M-\{0\}$ e paralelo com respeito à conexão de Chern.

Como consequência, podemos construir campos de Jacobi limitados ao longo de geodésicas sem pontos conjugados.

Corolário 4.9. Seja $(M, F)$ uma superfície Finsler k-básica sem pontos conjugados. Então uma e somente uma das condições abaixo vale:

- Jo definido acima é um campo de Jacobi limitado, perpendicular a $\gamma_{\theta}$ com respeito à métrica adaptada de $T M-\{0\}$, com $J_{\theta}(t) \neq 0$ para todo $t \in \mathbb{R}$.

- existe $t_{0} \in \mathbb{R}$ tal que $J_{\theta}\left(t_{0}\right)=0$. Neste caso, $J(t)=0$ para todo $t \in \mathbb{R} e$ consequentemente $i(t)=0$ para todo $t \in \mathbb{R}$.

Demonstração. Uma vez que o escalar de Cartan $I$ é uma função contínua e $\mathcal{S} M$ é compacto, $i(t)$ é limitada e portanto $J_{\theta}(t)$ é um campo de Jacobi limitado, perpendicular a $\gamma_{\theta}$ com respeito à métrica adaptada de $T M-\{0\}$. Se existe $t_{0} \in \mathbb{R}$ tal que $J_{\theta}\left(t_{0}\right)=0$, então pela Proposição $4.1 J_{\theta}$ deveria divergir, o que é impossível visto que $J_{\theta}(t)$ é limitado. Portanto $J_{\theta}(t)=0$ para todo $t$. Como $e(t) \neq 0$ para todo $t$, temos que $i(t)=0$ para todo $t \in \mathbb{R}$.

Cabe aqui fazer uma observação: o Corolário 4.7 nos diz que alguma órbita do fluxo geodésico é densa. Se formos capazes de construir ao longo desta órbita um campo de Jacobi do tipo $J_{\theta}(t)=i(t) e(t)$ que se anula em algum ponto, pelo corolário anterior teremos $i(t)=I\left(\gamma_{\theta}(t), \gamma_{\theta}^{\prime}(t)\right)=0$ em um conjunto denso de $\mathcal{S} M$. Da continuidade de $I$ segue que $I(\theta)=0$ para todo $\theta \in \mathcal{S} M$ e então a superfície é de fato Riemanniana.

De fato, nosso trabalho será tentar mostrar a existência de um campo de Jacobi do tipo $J_{\theta}(t)=i(t) e(t)$ que se anule sobre a órbita densa do fluxo geodésico. Para isso, começaremos estudando as propriedades do conjunto de pontos $\theta \in \mathcal{S} M$ para os quais o campo $J_{\theta}$ não se anula. 
Lema 4.10. Sejam $(M, F)$ uma superfície Finsler compacta, k-básica e sem pontos conjugado. Denote por $Y \subset \mathcal{S} M$ o conjunto dos pontos $\theta \in \mathcal{S} M$ tais que o campo de Jacobi $J_{\theta}$ dado pelo Lema 4.8 é não trivial ao longo de $\gamma_{\theta}$. Então,

1) $Y$ e seu complemento $Y^{\complement}$ são invariantes pelo fluxo geodésico,

2) $Y=\{\theta \in \mathcal{S} M ; I(\theta) \neq 0\}$,

3) Y é um conjunto aberto.

Demonstração. O item 1 seque do fato de $i(t)$ ser avaliada ao longo de uma órbita do fluxo geodésico e, pelo corolário anterior, $i(t)$ nunca se anula ou é sempre zero. O item 2 segue imediatamente da definição de $Y$. O item 3 segue do item 2 e da continuidade de $I$.

Definição 4.11. Um campo de Jacobi $J$ definido e limitado para todo $t \in \mathbb{R}$ é chamado campo de Jacobi central.

\section{3}

\section{Fibrados de Green, operadores de Ricatti e campos de Jacobi centrais}

Relembremos que dada uma superfície Finsler $(M, F)$, o fibrado unitário $\mathcal{S} M$ admite uma métrica Riemanniana de tipo Sasaki. Esta métrica é dada por

$$
g:=\omega^{1} \otimes \omega^{1}+\omega^{2} \otimes \omega^{2}+\omega^{3} \otimes \omega^{3}
$$

onde $\hat{e}_{i}, i=1,2,3$, é o referencial definido na página 23. Assim, dado $\theta \in \mathcal{S} M$, seja $N_{\theta}=H_{\theta} \oplus V_{\theta}$ o plano em $T_{\theta} T_{1} M$ que é perpendicular ao campo geodésico (o qual é tangente a $e_{2}$ ), onde $V_{\theta}$ é o subespaço vertical - tangente a $e_{3}-$ e $H_{\theta}$ é tangente a $e_{1}$.

Assim como na geometria Riemanniana, um campo de Jacobi definido ao longo de uma geodésica $\gamma_{\theta}$, perpendicular a esta com respeito à métrica adaptada, admite um levantamento natural $\left(J, J^{\prime}\right)$ em coordenadas horizontaisverticais para $T \tilde{M}$ ao longo da órbita $\phi_{t}(\theta)$ do fluxo geodésico.

Proposição 4.12 (Fibrados de Green). Seja $(M, F)$ uma superfície Finsler sem pontos conjugados. Então, para cada $\theta \in \mathcal{S} M$, existem dois subespaços invariantes unidimensionais $E^{s}(\theta)$ e $E^{u}(\theta)$ de $T_{\theta}(\mathcal{S} M)$, definidos por

$$
\begin{aligned}
& E^{s}(\theta):=\lim _{t \rightarrow+\infty} D \phi_{-t}\left(V_{\phi_{t}(\theta)}\right) \\
& E^{u}(\theta):=\lim _{t \rightarrow-\infty} D \phi_{-t}\left(V_{\phi_{t}(\theta)}\right)
\end{aligned}
$$

onde $D \phi_{t}$ é a derivada do fluxo geodésico e $V_{\theta}$ é o espaço vertical em $\theta$. As distribuições $E^{s}(\theta)$ e $E^{u}(\theta)$ têm as seguintes propriedades: 
1. Ambas são mensuráveis, transversais ao subfibrado vertical $V_{\theta}$ e transversais ao campo geodésico.

2. Existem operadores lineares $U_{\theta}^{s}: H_{\theta} \longrightarrow V_{\theta}$ e $U_{\theta}^{u}: H_{\theta} \longrightarrow V_{\theta}$ tais que $E^{s}(\theta)$ e $E^{u}(\theta)$ são respectivamente os gráficos de $U_{\theta}^{s}$ e $U_{\theta}^{u}$. Esses operadores, quando restritos a uma órbita do fluxo geodésico, são soluções da equação de Ricatti

$$
u^{\prime}(t)+u^{2}(t)+K(t)=0
$$

onde $K(t)=K\left(\phi_{t}(\theta)\right)$.

As distribuições $E^{s}(\theta)$ e $E^{u}(\theta)$ são chamadas respectivamente de fibrados de Green estável e instável e os operadores $U_{\theta}^{s}$ e $U_{\theta}^{u}$ são chamados operadores de Ricatti. Fibrados de Green foram definidos por Hopf e Green para métricas Riemannianas e por Foulon (veja [1], Section 2) para métricas Finsler. Para uma versão Hamiltoniana, veja por exemplo [30].

Enunciamos, sem a demonstração, uma conhecida relação entre fibrados de Green estáveis/instáveis e os campos de Jacobi estáveis/instáveis.

Lema 4.13. Sejam $J_{(\theta, V)}^{+}$e $J_{(\theta, V)}^{-}$campos de Jacobi estável e instável, respectivamente, ao longo de uma geodésica sem pontos conjugados $\gamma_{\theta}$, com $J_{(\theta, V)}^{+}(0)=J_{(\theta, V)}^{-}(0)=V$. Então $E^{s}(\theta)$ e $E^{u}(\theta)$ são gerados, respectivamente, pelos levantamentos

$$
\left(J_{(\theta, V)}^{+}(0), \dot{J}_{(\theta, V)}^{+}(0)\right) \quad e \quad\left(J_{(\theta, V)}^{-}(0), \dot{J}_{(\theta, V)}^{-}(0)\right) .
$$

Lema 4.14. Sejam $f, g: \mathbb{R} \longrightarrow \mathbb{R}$ duas soluções da equação escalar de Jacobi

$$
u^{\prime \prime}+K u=0
$$

sem pontos conjugados. Suponha que $f(0)=g(0)$ e $f^{\prime}(0)>g^{\prime}(0)$. Então, $f(t)>g(t)$ para todo $t>0$.

Demonstração. Se $f\left(t_{0}\right)=g\left(t_{0}\right)$ para algum $t_{0}>0$, então $h(t)=f(t)-g(t)$ é solução da equação escalar de Jacobi com $h(0)=h\left(t_{0}\right)=0$ e deve portanto se anular para todo $t$, pois não há pontos conjugados.

Aplicando o lema anterior e a Proposição 4.2, mostramos que todo campo de Jacobi central é na verdade Green-Jacobi estável e instável, um resultado análogo ao existente na geometria Riemanniana.

Lema 4.15. Um campo de Jacobi central ao longo de uma geodésica sem pontos conjugados é ao mesmo tempo Green-Jacobi estável e instável. 
Demonstração. Usaremos a divergência para $T>0$ de campos de Jacobi que se anulam em $t=0$ para mostrar que o campo de Jacobi central $J$ é GreenJacobi estável. A demonstração de que ele é também Green-Jacobi instável usa a divergência para $t<0$ (Corolário 4.2).

Suponha que $\|J(t)\| \leqslant C$ para todo $t$, onde $\|\cdot\|$ é a norma associada à métrica adaptada de $T M-\{0\}$. Seja $J_{T}$ o campo de Jacobi tal que

$$
J_{T}(0)=J(0) \quad \text { e } \quad J_{T}(T)=0 .
$$

Queremos mostra que para todo $t \in \mathbb{R}$ tem-se

$$
J(t)=\lim _{T \rightarrow \infty} J_{T}(t)
$$

Considere a família de campos de Jacobi dada por

$$
Y_{T}(t)=J_{T}(t)-J(t)
$$

que satisfaz

$$
Y_{T}(0)=0 \quad \text { e } \quad\left\|Y_{T}(T)\right\|=\|J(T)\| \leqslant C .
$$

Pela Proposição 4.1, $Y_{T}$ diverge para $t>0$. Em particular, para cada $T$, existe $t_{0}=t_{0}(T)>T$ tal que

$$
t \geqslant t_{0}(T) \quad \text { implica } \quad\left\|Y_{T}(t)\right\| \geqslant C .
$$

Afirmamos que $Y_{T}^{\prime}(0) \longrightarrow 0$ quando $T \longrightarrow \infty$. De fato, se tivéssemos

$$
\left\|Y_{T_{n}}^{\prime}(0)\right\| \nearrow \varepsilon
$$

para algum $\varepsilon>0$ e algum $T_{n} \longrightarrow \infty$, então fixado $T_{n_{0}}$,

$$
0<\left\|Y_{T_{n_{0}}}^{\prime}(0)\right\|<\left\|Y_{T_{n}}^{\prime}(0)\right\| \leqslant \varepsilon
$$

para todo $T_{n}>T_{n_{0}}$. Pelo Lema 4.14

$$
\left\|Y_{T_{n_{0}}}(t)\right\|<\left\|Y_{T_{n}}(t)\right\|
$$

para todo $t \geqslant 0$. Uma vez que $Y_{T_{n_{0}}}$ diverge, existe $\bar{t}_{0}$ tal que

$$
t \geqslant \bar{t}_{0} \quad \text { implica } \quad\left\|Y_{T_{n_{0}}}(t)\right\| \geqslant C \text {. }
$$

Escolhendo $T_{n}>\max \left\{\bar{t}_{0}, T_{n_{0}}\right\}$, temos por (4-6) que

$$
C \leqslant\left\|Y_{T_{n_{0}}}\left(T_{n}\right)\right\|<\left\|Y_{T_{n}}\left(T_{n}\right)\right\|
$$

o que contradiz (4-5). O caso $\left\|Y_{T_{n}}^{\prime}(0)\right\| \searrow \varepsilon$ é semelhante: escolha uma solução $J_{\varepsilon / 2}$ da equação de Jacobi satisfazendo 


$$
J_{\varepsilon / 2}(0)=0 \quad \text { e } \quad\left\|J_{\varepsilon / 2}^{\prime}(0)\right\|=\varepsilon / 2 .
$$

Pela Proposição 4.1, existe $t_{0} \in \mathbb{R}$ tal que

$$
t \geqslant t_{0} \quad \text { implica } \quad\left\|J_{\varepsilon / 2}(t)\right\| \geqslant C .
$$

Também, para todo $T_{n}$ vale que

$$
0<\left\|J_{\varepsilon / 2}^{\prime}(0)\right\|<\left\|Y_{T_{n}}^{\prime}(0)\right\|
$$

Pelo Lema 4.14,

$$
\left\|J_{\varepsilon / 2}(t)\right\|<\left\|Y_{T_{n}}(t)\right\|
$$

para todo $n$ e para todo $t>0$. Escolhendo $T_{n} \geqslant t_{0}$ temos que

$$
C \leqslant\left\|J_{\varepsilon / 2}\left(T_{n}\right)\right\|<\left\|Y_{T_{n}}\left(T_{n}\right)\right\|
$$

contradizendo (4-5) novamente e provando nossa afirmação. Agora com $Y_{T}^{\prime}(t)=$ $J_{T}^{\prime}(t)-J^{\prime}(t)$ e a afirmação, temos que

$$
\lim _{T \rightarrow \infty} J_{T}^{\prime}(0)=J^{\prime}(0)
$$

Como $J_{T}(0)=J(0)$ para todo $T>0$ nós temos que $J_{T}(t)$ converge uniformemente em $t$ para $J(t)$, pela dependência contínua das soluções da equação de Jacobi com respeito às condições iniciais. Portanto, $J$ é um campo de GreenJacobi estável.

\section{4}

\section{Fibrados de Green e o escalar de Cartan}

Como consequência do lema anterior, temos:

Corolário 4.16. Seja $(M, F)$ uma superfície Finsler compacta sem pontos conjugados. Dada uma geodésica $\gamma_{\theta}: \mathbb{R} \longrightarrow M$, o campo de Jacobi central

$$
J_{\theta}(t)=i(t) e(t)
$$

onde $i(t)=I\left(\gamma_{\theta}(t), \gamma_{\theta}^{\prime}(t)\right)$, é um campo de Green-Jacobi estável e instável ao longo de $\gamma_{\theta}$.

Lema 4.17. Seja $(M, F)$ uma superfície Finsler compacta sem pontos conjugados e de gênero maior que 1. Se $E^{s}(\theta)$ e $E^{u}(\theta)$ são transversais, então $I(\theta)=0$. Além disso,

$$
I\left(\phi^{t}(\theta)\right)=0 \text { para todo } t \in \mathbb{R}
$$


Demonstração. Se $I(\theta) \neq 0$, teríamos $i(t)=I\left(\gamma_{\theta}(t), \gamma_{\theta}^{\prime}(t)\right) \neq 0$ para todo $t$, pelo Lema 4.10. Então, $J_{\theta}(t)=i(t) e(t)$ seria um campo de Green-Jacobi estável e instável ao longo de $\gamma_{\theta}$, pelo Corolário 4.16. Logo, denotando $J_{\theta}(0)=V$, vemos que

$$
J_{(\theta, V)}^{+}(t)=J_{\theta}(t)=J_{(\theta, V)}^{-}(t)
$$

para todo $t \in \mathbb{R}$. Lembre que os fibrados estável e instável de Green no ponto $\theta$ são gerados respectivamente pelos levantamentos

$$
\left(J_{(\theta, V)}^{+}(0), \dot{J}_{(\theta, V)}^{+}(0)\right) \text { e }\left(J_{(\theta, V)}^{-}(0), \dot{J}_{(\theta, V)}^{-}(0)\right) .
$$

dos campos de Green-Jacobi estável e instável, respectivamente (veja o Lema 4.13). Em particular, avaliando os campos (4-8) em $t=0$ e usando (4-9), vemos que

$$
E^{s}(\theta)=E^{u}(\theta)
$$

uma contradição com a transversalidade. Portanto, $I(\theta)=0$ e pelo Lema 4.10, temos que $I\left(\phi^{t}(\theta)\right)=0$ para todo $t \in \mathbb{R}$.

Conforme observado na introdução desta tese e como deixa claro a demonstração do lema acima, nossos métodos dependem da bidimensionalidade.

Podemos ainda explorar interessantes relações entre campos de Jacobi centrais e o escalar de Cartan I. Como observado em [31],

Lema 4.18 (Gomes-Ruggiero, [31], Lemma 5.4). Seja $(M, F)$ uma superfície Finsler sem pontos conjugados. Então os fibrados de Green estável e instável coincidem em $Y$ e dependem continuamente de $\theta \in Y$.

Observando que $Y=\{\theta \in \mathcal{S} M / I(\theta) \neq 0\}$ é um aberto de $\mathcal{S} M$, a transitividade do fluxo geodésico (Corolário 4.7) nos fornece as seguintes consequências:

Corolário 4.19. Se $Y$ é não vazio, então $Y$ é denso em $\mathcal{S} M$.

Demonstração. Uma vez que $Y$ é aberto, a órbita densa do fluxo geodésico dada pelo Corolário 4.7 intersecta $Y$. O resultado segue da invariância de $Y$ pelo fluxo geodésico.

Corolário 4.20. Se $Y$ é não-vazio, $E^{s}$ e $E^{u}$ coincidem em um subconjunto aberto e denso de $\mathcal{S} M$.

Demonstração. Isto segue diretamente do Lema 4.17 e do Corolário 4.19 . 


\section{5 \\ Transitividade e rigidez em superfícies Finsler k-básicas}

Procederemos à demonstração do Teorema $\mathrm{A}$, enunciado no início deste capítulo. Primeiramente, relembramos um fato fundamental sobre dinâmica hiperbólica em dimensão 3.

Teorema 4.21 (Katok, [32]). Todo fluxo $C^{1+\alpha}$, com $\alpha>0$, em uma variedade de dimensão 3 com entropia topológica positiva tem uma órbita fechada hiperbólica com um ponto homoclínico transversal.

Um resultado devido a Dinaburg (ver [33], Theorem $4.1 \mathrm{ou}$, alternativamente, [34], Section 5.3.2) e que se estende para variedades Finsler (ver [18], pg. 424), garante que se o gênero de uma variedade Finsler compacta é maior que um, então a entropia topológica do fluxo geodésico é positiva. A hipótese do teorema de Dinaburg é que o grupo fundamental cresça exponencialmente, o que ocorre para superfícies compactas de gênero maior que um (Milnor provou em [35] que toda variedade que admite métrica Riemanniana de curvatura seccional negativa tem grupo fundamental de crescimento exponencial - ver também p.195 de [13]). Em verdade, todo grupo Gromov $\delta$-hiperbólico nãoelementar (ou seja, que não é finito e nem é extensão finita de um grupo cíclico infinito) tem crescimento uniformemente exponencial (Theorem 1.1, [12]). Pelo teorema de Katok, existe $\theta \in \mathcal{S} M$ tal que $\phi^{t}(\theta)$ é uma órbita hiperbólica fechada. Além disso, sobre uma órbita hiperbólica, os fibrados de Green $E^{s}$ e $E^{u}$ têm propriedades especiais, com enunciamos abaixo:

Lema 4.22 (Gomes-Ruggiero, [19], Lemma 5.2). Seja (M,F) uma superfície Finsler compacta sem pontos conjugados. Suponha que $\phi_{t}(\theta)$ é uma órbita hiperbólica do fluxo geodésico de $(M, F)$. Então os fibrados de Green $E^{s}(\theta)$ e $E^{u}(\theta)$ são respectivamente os subespaços estável e instável de $T_{\theta} T M$.

Finalmente apresentamos a prova do principal teorema do capítulo.

Teorema A. Seja $(M, F)$ uma superfície Finsler $k$-básica $C^{\infty}$ compacta, sem pontos conjugados, de gênero maior que 1 e cujos fibrados de Green são contínuos. Então $(M, F)$ é Riemanniana.

Demonstração. Pelo Teorema 1.10, basta provarmos que $I(\eta)=0$ para todo $\eta \in \mathcal{S} M$. Pelo Teorema 4.21, existe $\theta \in \mathcal{S} M$ tal que $\phi_{t}(\theta)$ é uma órbita hiperbólica do fluxo geodésico $\phi_{t}$. Então, pelo Lema 4.22, os fibrados de Green $E^{s}(\theta)$ e $E^{u}(\theta)$ são respectivamente os subespaços estáveis e instáveis dinâmicos e, consequentemente, são linearmente independentes. Pela continuidade dos fibrados de Green, existe um aberto $W \subset \mathcal{S} M$ contendo $\theta$ tal que $\eta \in W$ implica que $E^{s}(\eta)$ e $E^{u}(\eta)$ são linearmente independentes. Pelo Lema 4.17, 


$$
I(\eta)=0 \text { para todo } \eta \in W
$$

Combinando este fato com o Corolário 4.19 temos que o conjunto dos pontos onde $I \neq 0$ tem de ser vazio e assim, pela Proposição 1.10, a superfície Finsler $(M, F)$ é de fato Riemanniana, como queríamos provar. 


\section{Referências Bibliográficas}

[1] FOULON, P.. Estimation de l'entropie des systèmes lagrangiens sans points conjugués. 57(2):117-146, 1992.

[2] NIELSEN, J.. Om geodaetiske linier i lukkede mangfoldigheder med konstant negativ krumni. Matematisk Tidsskrift, p. 37-44, 1925.

[3] KOEBE, P.. Riemannsche mannigfaltigkeiten und nicht euklidische raumformen. Sitzungsberichte der Preussischen Akademie der Wissenschaften, p. I, 1927, pp. 164-196; II and III, 1928, pp. 345-442; IV, 1929, pp. 414-457; V and VI, 1930, pp. 304-364, 504-541; VII, 1931, pp. $506-534$.

[4] MORSE, M.; HEDLUND, G. A.. Manifolds without conjugate points. Transactions of the American Mathematical Society, 51:362-386, 1942.

[5] GREEN, L. W.. Surfaces without conjugate points. Transactions of the American Mathematical society, p. 529-546, 1954.

[6] EBERLEIN, P.. Geodesic flow in certain manifolds without conjugate points. Transactions of the American Mathematical Society, 167:151-170, 1972.

[7] EBERLEIN, P.; O'NEILL, B.. Visibility manifolds. Pacific Journal of Mathematics, 46(1):45-109, 1973.

[8] GROMOV, M.. Hyperbolic groups. Springer, 1987.

[9] BENAKLI, N.; KAPOVICH, I.. Boundaries of hyperbolic groups. Contemporary Mathematics, 296:39-94, 2002.

[10] KAPOVICH, M.. Lectures on the geometric group theory. preprint (as of September 28, 2005), 2005.

[11] COORnAERT, M.; PAPADOPOUlOS, A.. Symbolic dynamics and hyperbolic groups. Springer, 1993. 
[12] KOUBI, M.. Croissance uniforme dans les groupes hyperboliques. In: ANNALES DE L'INSTITUT FOURIER, volumen 48, p. 1441-1453, 1998.

[13] DE LA HARPE, P.. Topics in geometric group theory. University of Chicago Press, 2000.

[14] BRIDSON, M. R.; HAEFLIGER, A.. Metric spaces of non-positive curvature, volumen 319. Springer, 1999.

[15] AKBAR-ZADEH, H.. Champ de vecteurs projectifs sur le fibre unitaire. J. Math. Pures Appl, 65(1):986, 1986.

[16] BRYANT, R.. Finsler surfaces with prescribed curvature conditions. preprint, 1995.

[17] SHEN, Z.. Finsler manifolds with nonpositive flag curvature and constant s-curvature. Mathematische Zeitschrift, 249(3):625-639, 2005.

[18] PATERNAIN, G. P.. Finsler structures on surfaces with negative euler characteristic. Houston Journal of Mathematics, 23:421-426, 1997.

[19] GOMES, J. B.; RUGGIERO, R. O.. On finsler surfaces without conjugate points. Ergodic Theory and Dynamical Systems, 33(02):455474, 2013.

[20] BAO, D.; CHERN, S.-S. ; SHEN, Z.. An introduction to RiemannFinsler geometry, volumen 200. Springer Science \& Business Media, 2000 .

[21] ABATE, M.; PATRIZIO, G.. Finsler metrics: a global approach. Springer, 1994.

[22] RADEMACHER, H.-B.. A sphere theorem for non-reversible finsler metrics. Mathematische Annalen, 328(3):373-387, 2004.

[23] JAVALOYES, M. A.. Chern connection of a pseudo-finsler metric as a family of affine connections. arXiv preprint arXiv:1303.6263, 2013.

[24] RUGGIERO, R. O.. Expansive dynamics and hyperbolic geometry. Boletim da Sociedade Brasileira de Matemática-Bulletin/Brazilian Mathematical Society, 25(2):139-172, 1994. 
[25] PESIN, J. B.. Geodesic flows on closed riemannian manifolds without focal points. Mathematics of the USSR-Izvestiya, 11(6):1195, 1977.

[26] SHEN, Z.. Lectures on Finsler geometry, volumen 2001. World Scientific, 2001.

[27] Chimenton, Alessandro G; GOMES, J. B.; RUGGIERO, R. O.. Transitivity of finsler geodesic flows of compact surfaces without conjugate points and higher genus, and applications to finsler rigidity problems. Houston journal of mathematics, 41(2):523-551, 2015.

[28] BALLMANN, W.; BRIN, M. ; BURNS, K.. On surfaces with no conjugate points. Journal of Differential Geometry, 25:249-273, 1987.

[29] EBERLEIN, P.; OTHERS. When is a geodesic flow of anosov type? i. Journal of Differential Geometry, 8(3):437-463, 1973.

[30] CONTRERAS, G.; ITURRIAGA, R.. Convex hamiltonians without conjugate points. Ergodic Theory and Dynamical Systems, 19(04):901952, 1999.

[31] GOMES, J. B.; RUGGIERO, R. O.. Smooth k-basic finsler compact surfaces with expansive geodesic flows are riemannian. Houston journal of mathematics, 37(3):793-806, 2011.

[32] KATOK, A.. Lyapunov exponents, entropy and periodic orbits for diffeomorphisms. Inst. Hautes Etudes Sci. Publ. Math, 51(1):137-173, 1980.

[33] DINABURG, E. I.. On the relations among various entropy characteristics of dynamical systems. Izvestiya: Mathematics, 5(2):337-378, 1971.

[34] PATERnAIN, G.. Geodesic flows, volumen 180. Springer Science \& Business Media, 1999.

[35] MILNOR, J.; OTHERS. A note on curvature and fundamental group. Journal of Differential geometry, 2(1):1-7, 1968. 